\title{
Search for New Particles in Two-Jet Final States in 7 TeV Proton-Proton Collisions with the ATLAS Detector at the LHC
}

\author{
G. Aad et al.* \\ (ATLAS Collaboration) \\ (Received 13 August 2010; published 11 October 2010)
}

\begin{abstract}
A search for new heavy particles manifested as resonances in two-jet final states is presented. The data were produced in $7 \mathrm{TeV}$ proton-proton collisions by the LHC and correspond to an integrated luminosity of $315 \mathrm{nb}^{-1}$ collected by the ATLAS detector. No resonances were observed. Upper limits were set on the product of cross section and signal acceptance for excited-quark $\left(q^{*}\right)$ production as a function of $q^{*}$ mass. These exclude at the 95\% C.L. the $q^{*}$ mass interval $0.30<m_{q^{*}}<1.26 \mathrm{TeV}$, extending the reach of previous experiments.
\end{abstract}

PACS numbers: 13.85.Rm, 12.60.Rc, 13.87.Ce, 14.80.-j

Two-jet (dijet) events in high-energy proton-proton $(p p)$ collisions are usually described in the standard model (SM) by applying QCD to the scattering of beam-constituent quarks and gluons. Several extensions beyond the SM predict new heavy particles, accessible at LHC energies, that decay into two energetic partons. Such new states may include an excited composite quark $q^{*}$, exemplifying quark substructure [1-3], an axigluon predicted by chiral color models [4,5], a flavor-universal color-octet coloron [6,7], or a color-octet techni- $\rho$ meson predicted by models of extended technicolor and top-color-assisted technicolor [8-11].

Particularly sensitive to such new objects is the dijet invariant mass observable, defined as $m^{j j} \equiv$ $\sqrt{\left(E^{j_{1}}+E^{j_{2}}\right)^{2}-\left(\vec{p}^{j_{1}}+\vec{p}^{j_{2}}\right)^{2}}$, where $E$ and $\vec{p}$ are the jet energy and momentum, respectively. Several experiments have examined $m^{j j}$ distributions in search of new resonances [12-17]; recently, $1.13 \mathrm{fb}^{-1}$ of $p \bar{p}$ collision data at the Fermilab Tevatron collider have excluded the existence of excited quarks $q^{*}$ with mass $260<m_{q^{*}}<870 \mathrm{GeV}$ [16]. This Letter reports the first search by the ATLAS experiment [18] at the LHC for such massive particles in $p p$ collisions at a center-of-mass energy of $\sqrt{s}=7 \mathrm{TeV}$, based on a data sample corresponding to an integrated luminosity of $315 \mathrm{nb}^{-1}$. The analysis presented here focused on a search for excited quarks because of the accessible predicted cross section $[2,3]$ for such particles and the benchmark nature of the model that allows limits on the acceptance times cross section to be set for resonant states with intrinsic widths narrower than the experimental resolution.

*Full author list given at the end of the article.

Published by The American Physical Society under the terms of the Creative Commons Attribution 3.0 License. Further distribution of this work must maintain attribution to the author(s) and the published article's title, journal citation, and DOI.
The analysis technique consisted of a modelindependent search for a dijet mass resonance on top of a smooth and rapidly falling spectrum and relied on the measured $m^{j j}$ distribution to estimate the background level to this new possible signal. In the absence of an observed new physics signal, upper limits were determined on products of cross section $(\sigma)$ and signal acceptance $(\mathcal{A})$ for several $q^{*}$ test masses for a standard set of model parameters.

The ATLAS detector [18] is a multipurpose particle physics apparatus with a forward-backward symmetric cylindrical geometry and near $4 \pi$ coverage in solid angle [19]. The overall layout of the detector is dominated by its four superconducting magnet systems, which comprise a thin solenoid surrounding inner tracking detectors and three large toroids with an eightfold azimuthal symmetry.

The calorimeters, which are surrounded by an extensive muon system, are of particular importance to this analysis. In the pseudorapidity region $|\eta|<3.2$, high-granularity liquid-argon (LAr) electromagnetic sampling calorimeters are used. An iron-scintillator tile calorimeter provides hadronic coverage in the range $|\eta|<1.7$. The end-cap and forward regions, spanning $1.5<|\eta|<4$.9, are instrumented with LAr calorimetry for both electromagnetic and hadronic measurements.

The data sample was collected during stable periods of $7 \mathrm{TeV} p p$ collisions using a trigger configuration requiring the lowest-level hardware-based calorimeter jet trigger to satisfy a nominal transverse energy threshold of $15 \mathrm{GeV}$ [20]. This trigger had an efficiency greater than $99 \%$ for events with at least one jet with transverse energy higher than $80 \mathrm{GeV}$.

Jets were reconstructed by using the anti- $k_{T}$ jet clustering algorithm [21] with a radius parameter $R=0.6$. The inputs to this algorithm were clusters of calorimeter cells seeded by cells with energy significantly above the measured noise. Jet four-vectors were constructed by performing a four-vector sum over these cell clusters, treating each as an $(E, \vec{p})$ four-vector with zero mass. These were cor- 
rected for the effects of calorimeter noncompensation and inhomogeneities by using transverse-momentum $\left(p_{T}\right)$ and $\eta$-dependent calibration factors based on Monte Carlo (MC) corrections and validated with extensive test-beam and collision-data studies [20,22]. The $m^{j j}$ observable was computed without unfolding jets to hadrons or partons.

In order to suppress cosmic-ray and beam-related backgrounds, events were required to contain at least one primary collision vertex, defined by at least five reconstructed charged-particle tracks, each with a position, when extrapolated to the beam line, of $|z|<10 \mathrm{~cm}$. Events with at least two jets were retained if the highest $p_{T}$ jet (the "leading" jet) satisfied $p_{T}^{j_{1}}>80 \mathrm{GeV}$ and the next-toleading jet satisfied $p_{T}^{j_{2}}>30 \mathrm{GeV}$; this ensured that the data sample had high and unbiased trigger and jet reconstruction efficiencies. Those events containing a poorly measured jet with $p_{T}>15 \mathrm{GeV}$ were vetoed to prevent cases where a jet was incorrectly identified as one of the two leading jets [23]; this affected the event selection by less than $0.5 \%$. The two leading jets were required to satisfy several quality criteria [23] and to lie outside detector regions where the jet energy was not yet measured in an optimal way, such as the interval $1.3<\left|\eta^{\text {jet }}\right|<1.8$. Finally, both jets were required to be in the pseudorapidity region $\left|\eta^{\text {jet }}\right|<2.5$, and their pseudorapidity difference was required to satisfy $\left|\eta^{j_{1}}-\eta^{j_{2}}\right|<1.3$. These cuts, which suppress high-mass SM multijet background, were determined by performing an optimization of the potential signal from $q^{*}$ decays (using a $q^{*}$ mass of $1 \mathrm{TeV}$ ) compared with the SM background. There were 132433 candidates that satisfied these requirements.

The final event sample was selected by requiring the dijet invariant mass to satisfy $m^{j j}>200 \mathrm{GeV}$ in order to eliminate any potential kinematic bias in the $m^{j j}$ distributions from the selection requirements on the jet candidates. There were 37805 events in this sample, which formed the $m^{j j}$ distribution shown in Fig. 1.

MC signal events were generated by using the excitedquark $\left(q g \rightarrow q^{*}\right)$ production model [2,3]. The excited quark $q^{*}$ was assumed to have spin $1 / 2$ and quarklike couplings, relative to those of the SM $S U(2), U(1)$, and $S U$ (3) gauge groups, of $f=f^{\prime}=f_{s}=1$, respectively. The compositeness scale $(\Lambda)$ was set to the $q^{*}$ mass. Signal events were generated by using PYTHIA [24] 6.4.21, a leading-order parton-shower MC generator, with the modified leading-order MRST2007 [25] parton distribution functions (PDFs) and with the renormalization and factorization scales set to the mean $p_{T}$ of the two leading jets. PYTHIA was also used to decay the excited quarks to all possible SM final states, which were dominantly $q g$ but also $q W, q Z$, and $q \gamma$. The MC samples were produced [26] by using the ATLAS MC09 parameter tune [27] and a GEANT4-based detector simulation [28].

Figure 1 shows the predicted signals for $q^{*}$ masses of 500, 800, and $1200 \mathrm{GeV}$, after all selection cuts. The signal

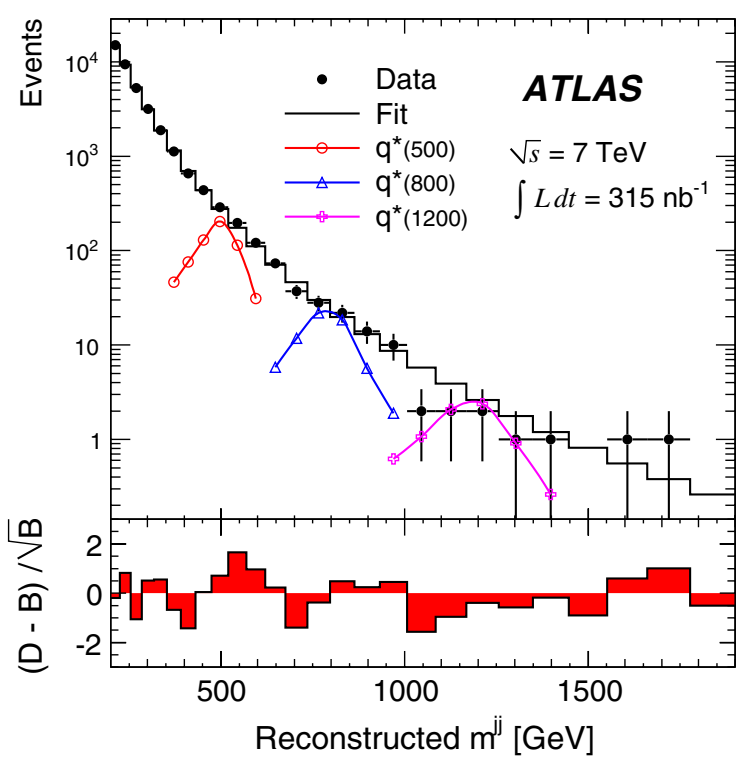

FIG. 1 (color online). The data $(D)$ dijet mass distribution (filled points) fitted by using a binned background $(B)$ distribution described by Eq. (1) (histogram). The predicted $q^{*}$ signals $[2,3]$ for excited-quark masses of 500, 800, and $1200 \mathrm{GeV}$ are overlaid, and the bin-by-bin significance of the data-background difference is shown.

acceptance $(\mathcal{A})$, which included reconstruction and trigger efficiencies near $100 \%$, was found to range from $\sim 31 \%$ for $m_{q^{*}}=300 \mathrm{GeV}$ to $\sim 48 \%$ for $m_{q^{*}}=1.7 \mathrm{TeV}$ [29]. The choice of dijet mass binning was motivated by the dijet mass resolution of the signal. The predicted experimental width ranged from $\sigma_{m^{j j}} / m^{j j} \sim 11 \%$ at $m_{q^{*}}=300 \mathrm{GeV}$ to $\sigma_{m^{j j}} / m^{j j} \sim 7 \%$ at $m_{q^{*}}=1.7 \mathrm{TeV}$ and was dominated by the detector energy resolution.

The background shape was determined by fitting the observed spectrum with the function [16]

$$
f(x)=p_{1}(1-x)^{p_{2}} x^{p_{3}+p_{4} \ln x},
$$

where $x \equiv m^{j j} / \sqrt{s}$, such that $f(1)=0$ and $f(0) \rightarrow+\infty$, and $p_{\{1,2,3,4\}}$ are free parameters. The $x^{p_{4} \ln x}$ factor was included to describe the high- $m^{j j}$ part of the spectrum. The function in Eq. (1) has been shown to fit the $m^{j j}$ observable well in PYTHIA, HERWIG, and next-to-leadingorder perturbative QCD predictions for $p \bar{p}$ collisions at $\sqrt{s}=1.96 \mathrm{TeV}$ [16]. Studies using PYTHIA and the ATLAS GEANT4-based detector simulation were performed to demonstrate that the smooth and monotonic form of Eq. (1) describes QCD-predicted dijet mass distributions in $p p$ collisions at $\sqrt{s}=7 \mathrm{TeV}$. There is good agreement between the MC prediction and the fitted parametrization in Eq. (1), as evidenced by a $\chi^{2}$ per degree of freedom of $27 / 22$ over the dijet mass range $200<m^{j j}<1900 \mathrm{GeV}$.

The results of fitting the data with Eq. (1) are shown in Fig. 1. The presence or absence of detectable $m^{j j}$ resonances in this distribution was determined by performing 
several statistical tests of the background-only hypothesis. A suite of six tests was employed: the BumpHunter [30], the Jeffreys divergence [31], the Kolmogorov-Smirnov test, the likelihood, the Pearson $\chi^{2}$, and the TailHunter statistic [32]. The agreement of the data with the background-only hypothesis of a smoothly varying and monotonic distribution was determined for each statistic by calculating the $p$ value for the data using $10^{3}$ pseudospectra drawn from Poisson variations seeded by the results of the fit of Eq. (1) to the data. The $p$ value of the background-only hypothesis is defined as the fraction of pseudoexperiments that result in a value of the given statistic greater than the value of the same statistic found by the fit to the data. The results of all six tests were consistent with the conclusion that the fitted parametrization described the observed data distribution well, with $p$ values in excess of $51 \%$. These observations supported the background-only hypothesis.

In the absence of any observed discrepancy with the zero-signal hypothesis, a Bayesian approach was used to set $95 \%$ credibility level (C.L.) upper limits on $\sigma \cdot \mathcal{A}$ for hypothetical new particles decaying into dijets with $\left|\eta^{\mathrm{jet}}\right|<2.5$. For each of the test masses (indexed by $\nu$ ) corresponding to the excited-quark $q^{*}$ predictions, a likelihood function $L_{\nu}$ was defined as a product of Poisson factors computed for each bin $(i)$ of the $m^{j j}$ distribution:

$$
L_{\nu}\left(d \mid b_{\nu}, s\right) \equiv \prod_{i} \frac{\left[b_{\nu i}+s_{i}(\nu)\right]^{d_{i}}}{d_{i} !} e^{-\left[b_{\nu i}+s_{i}(\nu)\right]},
$$

where $d_{i}$ is the observed number of data events in bin $i, b_{\nu i}$ is the background in bin $i$ obtained as described below, and $s_{i}(\nu)$ is the predicted signal added in bin $i$ by the signal template; the latter was normalized to the total number of predicted signal events $s=\sum_{i} s_{i}(\nu)$. For each $\nu$, the backgrounds in the bins $b_{\nu i}$ were evaluated from a simultaneous five-parameter fit of the signal and background distributions to ensure that the background determination would not be biased by the presence of any signal. The four background parameters were those in Eq. (1); the fifth parameter consisted of the normalization of the predicted $\nu^{\text {th }} q^{*}$ signal template. To avoid acceptance bias, the lowest $q^{*}$ test mass used was $300 \mathrm{GeV}$. For every $q^{*}$ mass, Eq. (2) was computed for a range of possible signal yields $s$, and the resulting likelihood function was multiplied by a flat prior in $s$ to give a posterior probability density in $s$. The 95\% probability region was then determined by integration of the posterior probability distribution. This Bayesian technique was found to yield credibility intervals that corresponded well with frequentist confidence intervals. This was verified by performing a series of pseudoexperiments to determine, by way of a standard frequentist calculation, the coverage, or the fraction of times that the 95\% Bayesian credibility interval contained the true number of signal events.
The dominant sources of systematic uncertainty, in decreasing order of importance, were the absolute jet energy scale, the background fit parameters, the integrated luminosity, and the jet energy resolution (JER). The jet energy scale uncertainty was quantified as a function of $p_{T}$ and $\eta^{\text {jet }}$, with values in the range $6 \%-9 \%[20,33,34]$. The jet calibration relied on the MC simulation of the response of the ATLAS detector; its uncertainty was constrained by varying the ATLAS simulation and from in situ information. The systematic uncertainty on the determination of the background was taken from the uncertainty on the parameters resulting from the fit of Eq. (1) to the data sample. The uncertainty on $\sigma \cdot \mathcal{A}$ due to integrated luminosity was estimated to be $\pm 11 \%$ [35]. The JER uncertainty was treated as uniform in $p_{T}$ and $\eta^{\text {jet }}$ with a value of $\pm 14 \%$ on the fractional $p_{T}$ resolution of each jet [36]. The effects of jet energy scale, background fit, integrated luminosity, and JER were incorporated as nuisance parameters into the likelihood function in Eq. (2) and then marginalized by numerically integrating the product of this modified likelihood, the prior in $s$, and the priors corresponding to the nuisance parameters to arrive at a modified posterior probability distribution. In the course of applying this convolution technique, the JER was found to make a negligible contribution to the overall systematic uncertainty.

Figure 2 depicts the resulting 95\% C.L. upper limits on $\sigma \cdot \mathcal{A}$ as a function of the $q^{*}$ resonance mass after incorporation of systematic uncertainties. Linear interpolations

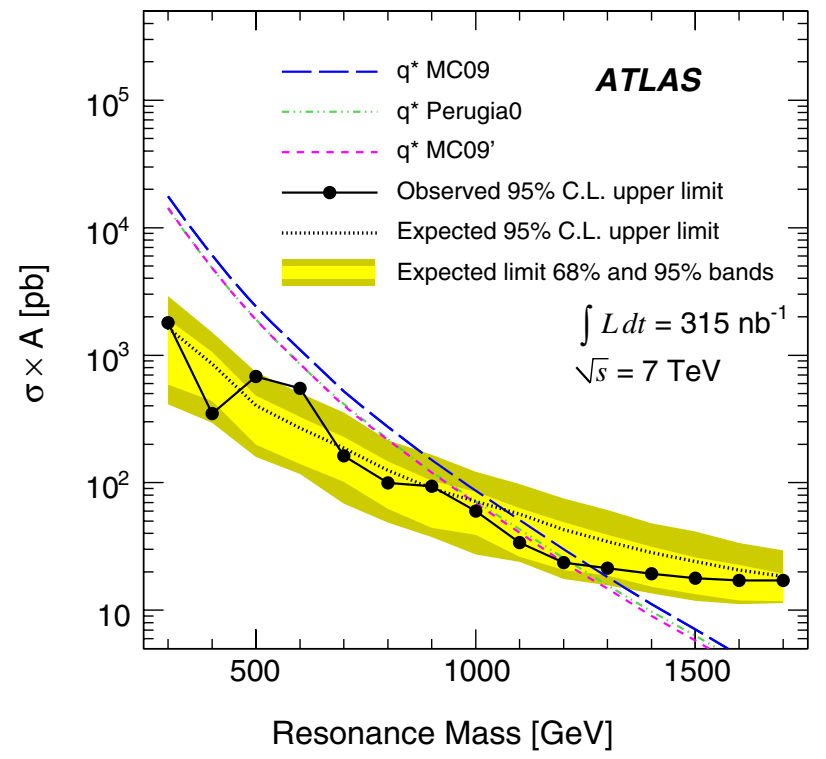

FIG. 2 (color online). The $95 \%$ C.L. upper limit on $\sigma \cdot \mathcal{A}$ as a function of dijet resonance mass (black filled circles). The black dotted curve shows the expected $95 \%$ C.L. upper limit, and the light and dark yellow shaded bands represent the 68\% and $95 \%$ credibility intervals of the expected limit, respectively. The dashed curves represent excited-quark $\sigma \cdot \mathcal{A}$ predictions for different MC tunes, each using a different PDF set. 
TABLE I. The $95 \%$ C.L. lower limits on the allowed $q^{*}$ mass obtained by using different PDF sets.

\begin{tabular}{lcccc}
\hline \hline MC tune & PDF set & \multicolumn{2}{c}{ Observed mass limit [TeV] } & Expected mass limit [TeV] \\
\hline MC09 [27] & MRST2007 [25] & 1.26 & 1.28 & 1.06 \\
MC09 ${ }^{\prime a}$ & CTEQ6L1 [37] & 1.20 & Stat. only & Stat. $\oplus$ Syst. \\
Perugia0 [38] & CTEQ5L [39] & 1.22 & 1.23 & 0.99 \\
\hline \hline
\end{tabular}

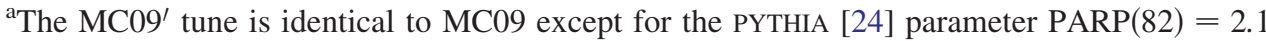
and use of the CTEQ6L1 PDF set.

between test masses were used to determine where the experimental bound intersected with a theoretical prediction to yield a lower limit on allowed mass. The corresponding observed $95 \%$ C.L. excited-quark mass exclusion region was found to be $0.30<m_{q^{*}}<1.26 \mathrm{TeV}$ by using MRST2007 PDFs in the ATLAS default MC09 tune. Table I shows the results obtained by using CTEQ6L1 [37] and CTEQ5L [39] PDF sets. The variations in the observed limit associated with the error eigenvectors of a CTEQ PDF set were found to be smaller than the spread displayed in Table I. The excluded regions were $\sim 30 \mathrm{GeV}$ greater when only statistical uncertainties were taken into account. The expected limits corresponding to the data sample were computed by using an analogous approach but replacing the actual data with pseudodata generated by random fluctuations around the smooth function described by fitting the data with Eq. (1); these are shown in Fig. 2, with a resulting expected $q^{*}$ mass exclusion region of $0.30<m_{q^{*}}<1.06 \mathrm{TeV}$ using MRST2007 PDFs. As indicated in Table I, the two other PDF sets yielded similar results, with expected exclusion regions extending to near $1 \mathrm{TeV}$. An indication of the dependence of the $m_{q^{*}}$ limits on the theoretical prediction for the $q^{*}$ signal was obtained by simultaneously varying both the renormalization and factorization scales by factors of 0.5 and 2 , which was tantamount to modifying the predicted cross section by approximately $\pm 20 \%$; this changed the observed MRST2007 limit of $1.26 \mathrm{TeV}$ to 1.32 and $1.22 \mathrm{TeV}$, respectively.

In conclusion, a model-independent search for new heavy particles manifested as mass resonances in dijet final states was conducted using a $315 \mathrm{nb}^{-1}$ sample of $7 \mathrm{TeV}$ proton-proton collisions produced by the LHC and recorded by the ATLAS detector. No evidence of a resonance structure was found, and upper limits at the $95 \%$ C.L. were set on the products of cross section and signal acceptance for hypothetical new $q^{*}$ particles decaying to dijets. These data exclude at the $95 \%$ C.L. excited-quark masses from the lower edge of the search region, $0.30 \mathrm{TeV}$, to $1.26 \mathrm{TeV}$ for a standard set of model parameters and using the ATLAS default MC09 tune [27]. This result extends the reach of previous experiments and constitutes the first exclusion of physics beyond the standard model by the ATLAS experiment. In the future, such searches will be extended to exclude or discover additional hypothetical particles over greater mass ranges.

We deeply thank everybody at CERN involved in operating the LHC in such a superb way during this initial highenergy data-taking period. We acknowledge equally warmly all the technical and administrative staff in the collaborating institutions without whom ATLAS could not be operated so efficiently. We acknowledge the support of ANPCyT, Argentina; Yerevan Physics Institute, Armenia; ARC and DEST, Australia; Bundesministerium für Wissenschaft und Forschung, Austria; National Academy of Sciences of Azerbaijan; State Committee on Science and Technologies of the Republic of Belarus; $\mathrm{CNPq}$ and FINEP, Brazil; NSERC, NRC, and CFI, Canada; CERN; CONICYT, Chile; NSFC, China; COLCIENCIAS, Colombia; Ministry of Education, Youth and Sports of the Czech Republic, Ministry of Industry and Trade of the Czech Republic, and Committee for Collaboration of the Czech Republic with CERN; Danish Natural Science Research Council and the Lundbeck Foundation; European Commission, through the ARTEMIS Research Training Network; IN2P3-CNRS and CEA-DSM/IRFU, France; Georgian Academy of Sciences; BMBF, DFG, HGF and MPG, Germany; Ministry of Education and Religion, through the EPEAEK program PYTHAGORAS II and GSRT, Greece; ISF, MINERVA, GIF, DIP, and Benoziyo Center, Israel; INFN, Italy; MEXT, Japan; CNRST, Morocco; FOM and NWO, The Netherlands; The Research Council of Norway; Ministry of Science and Higher Education, Poland; GRICES and FCT, Portugal; Ministry of Education and Research, Romania; Ministry of Education and Science of the Russian Federation and State Atomic Energy Corporation ROSATOM; JINR; Ministry of Science, Serbia; Department of International Science and Technology Cooperation, Ministry of Education of the Slovak Republic; Slovenian Research Agency, Ministry of Higher Education, Science and Technology, Slovenia; Ministerio de Educación y Ciencia, Spain; The Swedish Research Council, The Knut and Alice Wallenberg Foundation, Sweden; State Secretariat for Education and Science, Swiss National Science Foundation, and Cantons of Bern and Geneva, Switzerland; National Science Council, Taiwan; TAEK, Turkey; The Science and 
Technology Facilities Council and The Leverhulme Trust, United Kingdom; DOE and NSF, United States of America.

[1] E. Eichten, K. D. Lane, and M. E. Peskin, Phys. Rev. Lett. 50, 811 (1983).

[2] U. Baur, I. Hinchliffe, and D. Zeppenfeld, Int. J. Mod. Phys. A 2, 1285 (1987).

[3] U. Baur, M. Spira, and P. M. Zerwas, Phys. Rev. D 42, 815 (1990).

[4] P. H. Frampton and S. L. Glashow, Phys. Lett. B 190, 157 (1987).

[5] J. Bagger, C. Schmidt, and S. King, Phys. Rev. D 37, 1188 (1988).

[6] R. S. Chivukula, A. G. Cohen, and E. H. Simmons, Phys. Lett. B 380, 92 (1996).

[7] E. H. Simmons, Phys. Rev. D 55, 1678 (1997).

[8] K. D. Lane and M. V. Ramana, Phys. Rev. D 44, 2678 (1991).

[9] K. Lane and S. Mrenna, Phys. Rev. D 67, 115011 (2003).

[10] R. Foadi, M. T. Frandsen, T. A. Ryttov, and F. Sannino, Phys. Rev. D 76, 055005 (2007).

[11] A. Belyaev et al., Phys. Rev. D 79, 035006 (2009).

[12] J. Alitti et al. (UA2 Collaboration), Nucl. Phys. B400, 3 (1993).

[13] F. Abe et al. (CDF Collaboration), Phys. Rev. Lett. 74, 3538 (1995).

[14] F. Abe et al. (CDF Collaboration), Phys. Rev. D 55, R5263 (1997).

[15] V. Abazov et al. (D0 Collaboration), Phys. Rev. D 69, 111101 (2004).

[16] T. Aaltonen et al. (CDF Collaboration), Phys. Rev. D 79, 112002 (2009).

[17] F. Aaron et al. (H1 Collaboration), Phys. Lett. B 678, 335 (2009).

[18] ATLAS Collaboration, JINST 3, S08003 (2008).

[19] The ATLAS reference system is a Cartesian right-handed coordinate system, with the nominal collision point at the origin. The anticlockwise beam direction defines the positive $z$ axis, while the positive $x$ axis points from the collision point to the center of the LHC ring and the positive $y$ axis points upward. The angles $\phi$ and $\theta$ are the azimuthal and polar angles, respectively. The pseudorapidity is defined as $\eta=-\ln [\tan (\theta / 2)]$ and rapidity is defined as $y=\frac{1}{2} \ln \left[\left(E+p_{z}\right) /\left(E-p_{z}\right)\right]$, where $E$ is the energy and $p_{z}$ is the longitudinal component of the momentum along the beam direction.

[20] ATLAS Collaboration, Report No. ATLAS-CONF-2010050 (unpublished).

[21] M. Cacciari, G. P. Salam, and G. Soyez, J. High Energy Phys. 04 (2008) 063.

[22] E. Abat et al., Nucl. Instrum. Methods Phys. Res., Sect. A 621, 134 (2010).

[23] ATLAS Collaboration, Report No. ATLAS-CONF-2010038 (unpublished).

[24] T. Sjostrand, S. Mrenna, and P.Z. Skands, J. High Energy Phys. 05 (2006) 026.

[25] A. Sherstnev and R. S. Thorne, Eur. Phys. J. C 55, 553 (2008).

[26] ATLAS Collaboration, arXiv:1005.4568 [Eur. Phys. J. C. (to be published)].

[27] ATLAS Collaboration, Report No. ATL-PHYS-PUB2010-002 (unpublished).

[28] S. Agostinelli et al. (GEANT4 Collaboration), Nucl. Instrum. Methods Phys. Res., Sect. A 506, 250 (2003).

[29] For the specific $q g$ final state, the product of branching fraction and acceptance ranged from $\sim 28 \%$ to $\sim 40 \%$ for $m_{q^{*}}=300 \mathrm{GeV}$ and $m_{q^{*}}=1.7 \mathrm{TeV}$, respectively.

[30] T. Aaltonen et al. (CDF Collaboration), Phys. Rev. D 79, 011101 (2009).

[31] H. Jeffreys, Proc. R. Soc. A 186, 453 (1946).

[32] T. Aaltonen et al. (CDF Collaboration), Phys. Rev. D 78, 012002 (2008).

[33] ATLAS Collaboration, Report No. ATLAS-CONF-2010052 (unpublished).

[34] ATLAS Collaboration, Report No. ATLAS-CONF-2010056 (unpublished).

[35] ATLAS Collaboration, Report No. ATLAS-CONF-2010060 (unpublished).

[36] ATLAS Collaboration, Report No. ATLAS-CONF-2010054 (unpublished).

[37] J. Pumplin et al. (CTEQ Collaboration), J. High Energy Phys. 07 (2002) 012.

[38] P.Z. Skands, arXiv:0905.3418.

[39] H. L. Lai et al. (CTEQ Collaboration), Eur. Phys. J. C 12, 375 (2000).

G. Aad, ${ }^{48}$ B. Abbott, ${ }^{111}$ J. Abdallah, ${ }^{11}$ A. A. Abdelalim, ${ }^{49}$ A. Abdesselam, ${ }^{118}$ O. Abdinov, ${ }^{10}$ B. Abi, ${ }^{112}$ M. Abolins, ${ }^{88}$ H. Abramowicz, ${ }^{153}$ H. Abreu, ${ }^{115}$ E. Acerbi,${ }^{89 a, 89 b}$ B. S. Acharya,,${ }^{164 a, 164 b}$ M. Ackers, ${ }^{20}$ D. L. Adams, ${ }^{24}$ T. N. Addy, ${ }^{56}$ J. Adelman, ${ }^{175}$ M. Aderholz, ${ }^{99}$ S. Adomeit, ${ }^{98}$ C. Adorisio, ${ }^{36 a, 36 b}$ P. Adragna, ${ }^{75}$ T. Adye, ${ }^{129}$ S. Aefsky, ${ }^{22}$ J. A. Aguilar-Saavedra, ${ }^{124 b, b}$ M. Aharrouche, ${ }^{81}$ S. P. Ahlen, ${ }^{21}$ F. Ahles, ${ }^{48}$ A. Ahmad, ${ }^{148}$ H. Ahmed, ${ }^{2}$ M. Ahsan, ${ }^{40}$ G. Aielli, ${ }^{133 a, 133 b}$ T. Akdogan, ${ }^{18 a}$ T. P. A. Åkesson, ${ }^{79}$ G. Akimoto, ${ }^{155}$ A. V. Akimov, ${ }^{94}$ A. Aktas, ${ }^{48}$ M. S. Alam, ${ }^{1}$ M. A. Alam, ${ }^{76}$ S. Albrand, ${ }^{55}$ M. Aleksa, ${ }^{29}$ I. N. Aleksandrov, ${ }^{65}$ M. Aleppo,${ }^{89 a, 89 b}$ F. Alessandria, ${ }^{89 a}$ C. Alexa ${ }^{25 a}$ G. Alexander, ${ }^{153}$ G. Alexandre, ${ }^{49}$ T. Alexopoulos, ${ }^{9}$ M. Alhroob,${ }^{20}$ M. Aliev, ${ }^{15}$ G. Alimonti, ${ }^{89}$ J. Alison, ${ }^{120}$ M. Aliyev, ${ }^{10}$ P. P. Allport, ${ }^{73}$ S. E. Allwood-Spiers,${ }^{53}$ J. Almond, ${ }^{82}$ A. Aloisio, ${ }^{102 a, 102 b}$ R. Alon, ${ }^{171}$ A. Alonso, ${ }^{79}$ J. Alonso, ${ }^{14}$ M. G. Alviggi, ${ }^{102 a, 102 b}$ K. Amako, ${ }^{66}$ P. Amaral, ${ }^{29}$ G. Ambrosio, ${ }^{89 a, c}$ C. Amelung, ${ }^{22}$ V. V. Ammosov, ${ }^{128}$ A. Amorim, ${ }^{124 a, d}$ G. Amorós, ${ }^{167}$ N. Amram,,${ }^{153}$ C. Anastopoulos, ${ }^{139}$ T. Andeen, ${ }^{34}$ C. F. Anders, ${ }^{20}$ K. J. Anderson, ${ }^{30}$ A. Andreazza, ${ }^{89 a, 89 b}$ V. Andrei, ${ }^{58 a}$ M-L. Andrieux ${ }^{55}$ X. S. Anduaga, ${ }^{70}$ A. Angerami, ${ }^{34}$ F. Anghinolfi, ${ }^{29}$ N. Anjos, ${ }^{124 a}$ A. Annovi, ${ }^{47}$ A. Antonaki, ${ }^{8}$ M. Antonelli, ${ }^{47}$ S. Antonelli, ${ }^{19 a, 19 b}$ J. Antos, ${ }^{144 b}$ B. Antunovic, ${ }^{41}$ F. Anulli, ${ }^{132 a}$ S. Aoun, ${ }^{83}$ 
G. Arabidze, ${ }^{8}$ I. Aracena, ${ }^{143}$ Y. Arai ${ }^{66}$ A. T. H. Arce, ${ }^{44}$ J. P. Archambault, ${ }^{28}$ S. Arfaoui, ${ }^{29, e}$ J-F. Arguin, ${ }^{14}$ T. Argyropoulos, ${ }^{9}$ E. Arik, ${ }^{18 a, a}$ M. Arik, ${ }^{18 a}$ A. J. Armbruster, ${ }^{87}$ K. E. Arms, ${ }^{109}$ S. R. Armstrong, ${ }^{24}$ O. Arnaez, ${ }^{4}$ C. Arnault, ${ }^{115}$ A. Artamonov, ${ }^{95}$ D. Arutinov, ${ }^{20}$ M. Asai, ${ }^{143}$ S. Asai, ${ }^{155}$ R. Asfandiyarov, ${ }^{172}$ S. Ask,${ }^{27}$ B. Åsman, ${ }^{146 a, 146 b}$ D. Asner, ${ }^{28}$ L. Asquith, ${ }^{77}$ K. Assamagan, ${ }^{24}$ A. Astbury, ${ }^{169}$ A. Astvatsatourov,${ }^{52}$ G. Atoian, ${ }^{175}$ B. Aubert, ${ }^{4}$ B. Auerbach, ${ }^{175}$ E. Auge,${ }^{115}$ K. Augsten,,${ }^{127}$ M. Aurousseau, ${ }^{4}$ N. Austin, ${ }^{73}$ G. Avolio, ${ }^{163}$ R. Avramidou, ${ }^{9}$ D. Axen, ${ }^{168}$ C. Ay,${ }^{54}$ G. Azuelos, ${ }^{93, f}$ Y. Azuma, ${ }^{155}$ M. A. Baak, ${ }^{29}$ G. Baccaglioni, ${ }^{89 a}$ C. Bacci, ${ }^{134 a, 134 b}$ A. M. Bach, ${ }^{14}$ H. Bachacou, ${ }^{136}$ K. Bachas, ${ }^{29}$ G. Bachy, ${ }^{29}$ M. Backes, ${ }^{49}$ E. Badescu, ${ }^{25 a}$ P. Bagnaia, ${ }^{132 a, 132 b}$ Y. Bai, ${ }^{32 a}$ D. C. Bailey, ${ }^{158}$ T. Bain, ${ }^{158}$ J. T. Baines, ${ }^{129}$ O. K. Baker, ${ }^{175}$ M. D. Baker, ${ }^{24}$ S Baker, ${ }^{77}$ F. Baltasar Dos Santos Pedrosa, ${ }^{29}$ E. Banas, ${ }^{38}$ P. Banerjee, ${ }^{93}$ S. Banerjee, ${ }^{169}$ D. Banfi, ${ }^{89 a, 89 b}$ A. Bangert, ${ }^{137}$ V. Bansal, ${ }^{169}$ S. P. Baranov, ${ }^{94}$ S. Baranov, ${ }^{65}$ A. Barashkou, ${ }^{65}$ A. Barbaro Galtieri, ${ }^{14}$ T. Barber, ${ }^{27}$ E. L. Barberio, ${ }^{86}$ D. Barberis,${ }^{50,50 b}$ M. Barbero, ${ }^{20}$ D. Y. Bardin, ${ }^{65}$ T. Barillari, ${ }^{99}$ M. Barisonzi, ${ }^{174}$ T. Barklow, ${ }^{143}$ N. Barlow, ${ }^{27}$ B. M. Barnett, ${ }^{129}$ R. M. Barnett, ${ }^{14}$ A. Baroncelli, ${ }^{134 a}$ M. Barone, ${ }^{47}$ A. J. Barr, ${ }^{118}$ F. Barreiro ${ }^{80}$ J. Barreiro Guimarães da Costa,${ }^{57}$ P. Barrillon, ${ }^{115}$ R. Bartoldus, ${ }^{143}$ D. Bartsch ${ }^{20}$ R. L. Bates ${ }^{53}$ L. Batkova, ${ }^{144 a}$ J. R. Batley,${ }^{27}$ A. Battaglia,${ }^{16}$ M. Battistin, ${ }^{29}$ G. Battistoni, ${ }^{89 a}$ F. Bauer, ${ }^{136}$ H. S. Bawa, ${ }^{143}$ M. Bazalova, ${ }^{125}$ B. Beare,${ }^{158}$ T. Beau, ${ }^{78}$ P. H. Beauchemin,${ }^{118}$ R. Beccherle,${ }^{50 a}$ P. Bechtle,${ }^{41}$ G. A. Beck, ${ }^{75}$ H. P. Beck, ${ }^{16}$ M. Beckingham, ${ }^{48}$ K. H. Becks, ${ }^{174}$ A. J. Beddall, ${ }^{18 c}$ A. Beddall, ${ }^{18 c}$ V. A. Bednyakov, ${ }^{65}$ C. Bee,${ }^{83}$ M. Begel,${ }^{24}$ S. Behar Harpaz,${ }^{152}$ P. K. Behera, ${ }^{63}$ M. Beimforde,,${ }^{99}$ C. Belanger-Champagne, ${ }^{166}$ B. Belhorma,${ }^{55}$ P. J. Bell, ${ }^{49}$ W. H. Bell, ${ }^{49}$ G. Bella,${ }^{153}$ L. Bellagamba,${ }^{19 a}$ F. Bellina, ${ }^{29}$ G. Bellomo, ${ }^{89 a, 89 b}$ M. Bellomo, ${ }^{119 a}$ A. Belloni, ${ }^{57}$ K. Belotskiy, ${ }^{96} \mathrm{O}$. Beltramello, ${ }^{29} \mathrm{~S}$. Ben Ami, ${ }^{152}$ O. Benary, ${ }^{153}$ D. Benchekroun, ${ }^{135 a}$ C. Benchouk, ${ }^{83}$ M. Bendel,${ }^{81}$ B. H. Benedict,${ }^{163}$ N. Benekos, ${ }^{165}$ Y. Benhammou, ${ }^{153}$ G. P. Benincasa, ${ }^{124 a}$ D. P. Benjamin, ${ }^{44}$ M. Benoit, ${ }^{115}$ J. R. Bensinger ${ }^{22}$ K. Benslama, ${ }^{130}$ S. Bentvelsen, ${ }^{105}$ M. Beretta, ${ }^{47}$ D. Berge,${ }^{29}$ E. Bergeaas Kuutmann, ${ }^{41}$ N. Berger, ${ }^{4}$ F. Berghaus, ${ }^{169}$ E. Berglund, ${ }^{49}$ J. Beringer, ${ }^{14}$ K. Bernardet,${ }^{83}$ P. Bernat, ${ }^{115}$ R. Bernhard, ${ }^{48}$ C. Bernius, ${ }^{77}$ T. Berry,${ }^{76}$ A. Bertin, ${ }^{19 a, 19 b}$ F. Bertinelli, ${ }^{29}$ F. Bertolucci, ${ }^{122 a, 122 b}$ S. Bertolucci, ${ }^{47}$ M. I. Besana, ${ }^{89 a, 89 b}$ N. Besson, ${ }^{136}$ S. Bethke, ${ }^{99}$ W. Bhimji, ${ }^{45}$ R. M. Bianchi,${ }^{48}$ M. Bianco, ${ }^{72 a, 72 b}$ O. Biebel,,${ }^{98}$ J. Biesiada, ${ }^{14}$ M. Biglietti, ${ }^{132 a, 132 b}$ H. Bilokon,${ }^{47}$ M. Binder, ${ }^{98}$ M. Bindi, ${ }^{19 a, 19 b}$ S. Binet,${ }^{115}$ A. Bingul, ${ }^{18 c}$ C. Bini, ${ }^{132 a, 132 b}$ C. Biscarat, ${ }^{177}$ R. Bischof,${ }^{62}$ U. Bitenc,${ }^{48}$ K. M. Black,${ }^{57}$ R. E. Blair, ${ }^{5}$ J-B Blanchard, ${ }^{115}$ G. Blanchot, ${ }^{29}$ C. Blocker, ${ }^{22}$ J. Blocki, ${ }^{38}$ A. Blondel, ${ }^{49}$ W. Blum, ${ }^{81}$ U. Blumenschein, ${ }^{54}$ C. Boaretto, ${ }^{132 a, 132 b}$ G. J. Bobbink, ${ }^{105}$ A. Bocci, ${ }^{44}$ D. Bocian, ${ }^{38}$ R. Bock,${ }^{29}$ C. R. Boddy,,${ }^{118}$ M. Boehler, ${ }^{41}$ J. Boek,${ }^{174}$ N. Boelaert, ${ }^{79}$ S. Böser, ${ }^{77}$ J. A. Bogaerts, ${ }^{29}$ A. Bogouch, ${ }^{90, a}$ C. Bohm, ${ }^{146 a}$ J. Bohm, ${ }^{125}$ V. Boisvert, ${ }^{76}$ T. Bold, ${ }^{163, g}$ V. Boldea, ${ }^{25 a}$ V. G. Bondarenko, ${ }^{96}$ M. Bondioli, ${ }^{163}$ M. Boonekamp, ${ }^{136}$ G. Boorman, ${ }^{76}$ C. N. Booth, ${ }^{139}$ P. Booth, ${ }^{139}$ J. R. A. Booth, ${ }^{17}$

S. Bordoni ${ }^{78}$ C. Borer,${ }^{16}$ A. Borisov, ${ }^{128}$ G. Borissov,${ }^{71}$ I. Borjanovic, ${ }^{12 a}$ S. Borroni, ${ }^{132 a, 132 b}$ K. Bos,${ }^{105}$

D. Boscherini, ${ }^{19 a}$ M. Bosman, ${ }^{11}$ H. Boterenbrood, ${ }^{105}$ D. Botterill, ${ }^{129} \mathrm{~J}$. Bouchami, ${ }^{93} \mathrm{~J}$. Boudreau,${ }^{123}$

E. V. Bouhova-Thacker ${ }^{71}$ C. Boulahouache, ${ }^{123}$ C. Bourdarios, ${ }^{115}$ A. Boveia ${ }^{30}$ J. Boyd ${ }^{29}$ I. R. Boyko, ${ }^{65}$

N. I. Bozhko, ${ }^{128}$ I. Bozovic-Jelisavcic, ${ }^{12 b}$ S. Braccini, ${ }^{47}$ J. Bracinik,,${ }^{17}$ A. Braem,${ }^{29}$ E. Brambilla, ${ }^{72 a, 72 b}$ P. Branchini, ${ }^{134 a}$ G. W. Brandenburg, ${ }^{57}$ A. Brandt, ${ }^{7}$ G. Brandt, ${ }^{41}$ O. Brandt, ${ }^{54}$ U. Bratzler, ${ }^{156}$ B. Brau, ${ }^{84}$ J. E. Brau, ${ }^{114}$ H. M. Braun, ${ }^{174}$ B. Brelier, ${ }^{158}$ J. Bremer, ${ }^{29}$ R. Brenner, ${ }^{166}$ S. Bressler, ${ }^{152}$ D. Breton, ${ }^{115}$ N. D. Brett, ${ }^{118}$ P. G. Bright-Thomas, ${ }^{17}$ D. Britton, ${ }^{53}$ F. M. Brochu, ${ }^{27}$ I. Brock, ${ }^{20}$ R. Brock, ${ }^{88}$ T. J. Brodbeck, ${ }^{71}$ E. Brodet, ${ }^{153}$ F. Broggi, ${ }^{89 a}$ C. Bromberg, ${ }^{88}$ G. Brooijmans, ${ }^{34}$ W. K. Brooks, ${ }^{31 b}$ G. Brown,${ }^{82}$ E. Brubaker, ${ }^{30}$

P. A. Bruckman de Renstrom, ${ }^{38}$ D. Bruncko, ${ }^{144 \mathrm{~b}}$ R. Bruneliere, ${ }^{48}$ S. Brunet, ${ }^{61}$ A. Bruni, ${ }^{19 a}$ G. Bruni, ${ }^{19 a}$ M. Bruschi, ${ }^{19 a}$ T. Buanes,${ }^{13}$ F. Bucci, ${ }^{49}$ J. Buchanan, ${ }^{118}$ N. J. Buchanan, ${ }^{2}$ P. Buchholz, ${ }^{141}$ R. M. Buckingham, ${ }^{118}$ A. G. Buckley, ${ }^{45}$ I. A. Budagov, ${ }^{65}$ B. Budick, ${ }^{108}$ V. Büscher, ${ }^{81}$ L. Bugge, ${ }^{117}$ D. Buira-Clark, ${ }^{118}$ E. J. Buis,${ }^{105}$ O. Bulekov, ${ }^{96}$ M. Bunse, ${ }^{42}$ T. Buran,${ }^{117}$ H. Burckhart ${ }^{29}$ S. Burdin,${ }^{73}$ T. Burgess, ${ }^{13}$ S. Burke, ${ }^{129}$ E. Busato, ${ }^{33}$ P. Bussey, ${ }^{53}$ C. P. Buszello, ${ }^{166}$ F. Butin, ${ }^{29}$ B. Butler, ${ }^{143}$ J. M. Butler,${ }^{21}$ C. M. Buttar,${ }^{53}$ J. M. Butterworth, ${ }^{77}$ T. Byatt, ${ }^{77}$ J. Caballero, ${ }^{24}$ S. Cabrera Urbán, ${ }^{167}$ M. Caccia,${ }^{89 a, 89 b, h}$ D. Caforio, ${ }^{19 a, 19 b}$ O. Cakir, ${ }^{3 a}$ P. Calafiura, ${ }^{14}$ G. Calderini, ${ }^{78}$ P. Calfayan, ${ }^{98}$ R. Calkins, ${ }^{106}$ L. P. Caloba, ${ }^{23}$ R. Caloi, ${ }^{132 a, 132 b}$ D. Calvet, ${ }^{33}$ A. Camard, ${ }^{78}$ P. Camarri, ${ }^{133 a, 133 b}$ M. Cambiaghi, ${ }^{119 a, 119 b}$ D. Cameron, ${ }^{117}$ J. Cammin, ${ }^{20}$ S. Campana, ${ }^{29}$ M. Campanelli, ${ }^{77}$ V. Canale, ${ }^{102 a, 102 b}$ F. Canelli, ${ }^{30}$ A. Canepa, ${ }^{159 a}$ J. Cantero, ${ }^{80}$ L. Capasso, ${ }^{102 a, 102 b}$ M. D. M. Capeans Garrido, ${ }^{29}$ I. Caprini, ${ }^{25 a}$ M. Caprini, ${ }^{25 a}$ M. Caprio, ${ }^{102 a, 102 b}$ D. Capriotti, ${ }^{99}$ M. Capua, ${ }^{36 a, 36 b}$ R. Caputo, ${ }^{148}$ C. Caramarcu, ${ }^{25 a}$ R. Cardarelli, ${ }^{133 a}$ T. Carli, ${ }^{29}$ G. Carlino, ${ }^{102 a}$ L. Carminati, ${ }^{89 a, 89 b}$ B. Caron,,${ }^{2, f}$ S. Caron, ${ }^{48}$ C. Carpentieri, ${ }^{48}$ G. D. Carrillo Montoya, ${ }^{172}$ S. Carron Montero, ${ }^{158}$ A. A. Carter,${ }^{75}$ J. R. Carter, ${ }^{27}$ J. Carvalho, ${ }^{124 a, i}$ D. Casadei,${ }^{108}$ M. P. Casado, ${ }^{11}$ M. Cascella, ${ }^{122 a, 122 b}$ C. Caso, ${ }^{50,50 b, a}$ A. M. Castaneda Hernandez, ${ }^{172}$ E. Castaneda-Miranda, ${ }^{172}$ V. Castillo Gimenez, ${ }^{167}$ N. F. Castro, ${ }^{124 b, b}$ G. Cataldi, ${ }^{72 a}$ F. Cataneo,${ }^{29}$ A. Catinaccio, ${ }^{29}$ J. R. Catmore, ${ }^{71}$ A. Cattai, ${ }^{29}$ 
G. Cattani, ${ }^{133 a, 133 b}$ S. Caughron, ${ }^{34}$ D. Cauz, ${ }^{164 a, 164 c}$ A. Cavallari, ${ }^{132 a, 132 b}$ P. Cavalleri, ${ }^{78}$ D. Cavalli, ${ }^{89 a}$ M. Cavalli-Sforza, ${ }^{11}$ V. Cavasinni, ${ }^{122 a, 122 b}$ A. Cazzato, ${ }^{72 a, 72 b}$ F. Ceradini,${ }^{134 a, 134 b}$ C. Cerna ${ }^{83}$ A. S. Cerqueira, ${ }^{23}$ A. Cerri, ${ }^{29}$ L. Cerrito, ${ }^{75}$ F. Cerutti, ${ }^{47}$ M. Cervetto, ${ }^{50 a, 50 b}$ S. A. Cetin, ${ }^{18 b}$ F. Cevenini, ${ }^{102 a, 102 b}$ A. Chafaq, ${ }^{135 a}$ D. Chakraborty, ${ }^{106}$ K. Chan, ${ }^{2}$ J. D. Chapman, ${ }^{27}$ J. W. Chapman, ${ }^{87}$ E. Chareyre, ${ }^{78}$ D. G. Charlton, ${ }^{17}$ V. Chavda, ${ }^{82}$ S. Cheatham, ${ }^{71}$ S. Chekanov, ${ }^{5}$ S. V. Chekulaev, ${ }^{159 a}$ G. A. Chelkov, ${ }^{65}$ H. Chen,${ }^{24}$ L. Chen, ${ }^{2}$ S. Chen, ${ }^{32 \mathrm{c}}$ T. Chen, ${ }^{32 \mathrm{c}}$ X. Chen, ${ }^{172}$ S. Cheng, ${ }^{32 a}$ A. Cheplakov, ${ }^{65}$ V. F. Chepurnov ${ }^{65}$ R. Cherkaoui El Moursli, ${ }^{135 c}$ V. Tcherniatine, ${ }^{24}$ D. Chesneanu, ${ }^{25 a}$ E. Cheu, ${ }^{6}$ S. L. Cheung, ${ }^{158}$ L. Chevalier, ${ }^{136}$ F. Chevallier, ${ }^{136}$ V. Chiarella, ${ }^{47}$ G. Chiefari, ${ }^{102 a, 102 b}$ L. Chikovani ${ }^{51}$ J. T. Childers, ${ }^{58 a}$ A. Chilingarov, ${ }^{71}$ G. Chiodini, ${ }^{72 a}$ M. V. Chizhov,${ }^{65}$ G. Choudalakis, ${ }^{30}$

S. Chouridou, ${ }^{137}$ I. A. Christidi, ${ }^{77}$ A. Christov, ${ }^{48}$ D. Chromek-Burckhart, ${ }^{29}$ M. L. Chu, ${ }^{151}$ J. Chudoba, ${ }^{125}$ G. Ciapetti, ${ }^{132 a, 132 b}$ A. K. Ciftci, ${ }^{3 a}$ R. Ciftci, ${ }^{3 a}$ D. Cinca, ${ }^{33}$ V. Cindro, ${ }^{74}$ M. D. Ciobotaru, ${ }^{163}$ C. Ciocca, ${ }^{19 a, 19 b}$ A. Ciocio, ${ }^{14}$ M. Cirilli, ${ }^{87, j}$ M. Citterio, ${ }^{89 a}$ A. Clark, ${ }^{49}$ P. J. Clark, ${ }^{45}$ W. Cleland, ${ }^{123}$ J. C. Clemens, ${ }^{83}$ B. Clement ${ }^{55}$ C. Clement, ${ }^{146 a, 146 b}$ R. W. Clifft, ${ }^{129}$ Y. Coadou, ${ }^{83}$ M. Cobal,${ }^{164 a, 164 c}$ A. Coccaro,${ }^{50 a, 50 b}$ J. Cochran, ${ }^{64}$ P. Coe, ${ }^{118}$ S. Coelli, ${ }^{89 a}$ J. Coggeshall, ${ }^{165}$ E. Cogneras, ${ }^{177}$ C. D. Cojocaru, ${ }^{28}$ J. Colas, ${ }^{4}$ B. Cole, ${ }^{34}$ A. P. Colijn, ${ }^{105}$ C. Collard, ${ }^{115}$ N. J. Collins, ${ }^{17}$ C. Collins-Tooth, ${ }^{53}$ J. Collot, ${ }^{55}$ G. Colon,${ }^{84}$ R. Coluccia, ${ }^{72 a, 72 b}$ G. Comune, ${ }^{88}$ P. Conde Muiño, ${ }^{124 a}$ E. Coniavitis, ${ }^{118}$ M. C. Conidi, ${ }^{11}$ M. Consonni, ${ }^{104}$ S. Constantinescu, ${ }^{25 a}$ C. Conta, ${ }^{119 a, 119 b}$ F. Conventi, ${ }^{102 a, k}$

J. Cook, ${ }^{29}$ M. Cooke, ${ }^{34}$ B. D. Cooper, ${ }^{75}$ A. M. Cooper-Sarkar, ${ }^{18}$ N. J. Cooper-Smith, ${ }^{76}$ K. Copic, ${ }^{34}$

T. Cornelissen, ${ }^{50 a, 50 b}$ M. Corradi, ${ }^{19 a}$ S. Correard, ${ }^{83}$ F. Corriveau, ${ }^{85,1}$ A. Corso-Radu, ${ }^{163}$ A. Cortes-Gonzalez, ${ }^{165}$ G. Cortiana, ${ }^{99}$ G. Costa, ${ }^{89 a}$ M. J. Costa, ${ }^{167}$ D. Costanzo, ${ }^{139}$ T. Costin,${ }^{30}$ D. Côté, ${ }^{29}$ R. Coura Torres,${ }^{23}$ L. Courneyea, ${ }^{169}$ G. Cowan, ${ }^{76}$ C. Cowden, ${ }^{27}$ B. E. Cox,${ }^{82}$ K. Cranmer, ${ }^{108}$ J. Cranshaw, ${ }^{5}$ M. Cristinziani, ${ }^{20}$ G. Crosetti, ${ }^{36 a, 36 b}$ R. Crupi, ${ }^{72 a, 72 b}$ S. Crépé-Renaudin, ${ }^{55}$ C. Cuenca Almenar, ${ }^{175}$ T. Cuhadar Donszelmann, ${ }^{139}$ S. Cuneo, ${ }^{50 a, 50 b}$ M. Curatolo, ${ }^{47}$ C. J. Curtis, ${ }^{17}$ P. Cwetanski, ${ }^{61}$ H. Czirr, ${ }^{141}$ Z. Czyczula, ${ }^{175}$ S. D'Auria,${ }^{53}$ M. D’Onofrio, ${ }^{73}$ A. D’Orazio, ${ }^{99}$ A. Da Rocha Gesualdi Mello, ${ }^{23}$ P. V. M. Da Silva, ${ }^{23}$ C Da Via, ${ }^{82}$ W. Dabrowski, ${ }^{37}$ A. Dahlhoff, ${ }^{48}$ T. Dai, ${ }^{87}$ C. Dallapiccola,${ }^{84}$ S. J. Dallison, ${ }^{129, a}$ J. Dalmau, ${ }^{75}$ C. H. Daly, ${ }^{138}$ M. Dam,${ }^{35}$ M. Dameri, ${ }^{50,50 b}$ H. O. Danielsson, ${ }^{29}$ R. Dankers, ${ }^{105}$ D. Dannheim, ${ }^{99}$ V. Dao, ${ }^{49}$ G. Darbo, ${ }^{50 a}$ G. L. Darlea, ${ }^{25 b}$ C. Daum, ${ }^{105}$ J. P. Dauvergne, ${ }^{29}$ W. Davey,${ }^{86}$ T. Davidek, ${ }^{126}$ N. Davidson, ${ }^{86}$ R. Davidson,,${ }^{71}$ M. Davies,${ }^{93}$ A. R. Davison, ${ }^{77}$ E. Dawe, ${ }^{142}$ I. Dawson, ${ }^{139}$ J. W. Dawson, ${ }^{5}$ R. K. Daya,${ }^{39}$ K. De,${ }^{7}$ R. de Asmundis, ${ }^{102 a}$ S. De Castro, ${ }^{19 a, 19 b}$ P. E. De Castro Faria Salgado, ${ }^{24}$ S. De Cecco,${ }^{78}$ J. de Graat,${ }^{98}$ N. De Groot, ${ }^{104}$ P. de Jong, ${ }^{105}$ E. De La Cruz-Burelo, ${ }^{87}$ C. De La Taille, ${ }^{115}$ B. De Lotto, ${ }^{164 a, 164 c}$ L. De Mora, ${ }^{71}$ L. De Nooij, ${ }^{105}$

M. De Oliveira Branco, ${ }^{29}$ D. De Pedis, ${ }^{132 a}$ P. de Saintignon, ${ }^{55}$ A. De Salvo, ${ }^{132 a}$ U. De Sanctis, ${ }^{164 a, 164 c}$ A. De Santo, ${ }^{149}$

J. B. De Vivie De Regie, ${ }^{115}$ G. De Zorzi, ${ }^{132 a, 132 b}$ S. Dean,${ }^{77}$ G. Dedes,${ }^{99}$ D. V. Dedovich, ${ }^{65}$ P. O. Defay, ${ }^{33}$

J. Degenhardt, ${ }^{120}$ M. Dehchar ${ }^{118}$ M. Deile, ${ }^{98}$ C. Del Papa,${ }^{164 a, 164 c}$ J. Del Peso, ${ }^{80}$ T. Del Prete, ${ }^{122 a, 122 b}$

A. Dell'Acqua, ${ }^{29}$ L. Dell'Asta ${ }^{89 a, 89 b}$ M. Della Pietra, ${ }^{102 a, k}$ D. della Volpe,${ }^{102 a, 102 b}$ M. Delmastro, ${ }^{29}$ P. Delpierre,${ }^{83}$ N. Delruelle, ${ }^{29}$ P. A. Delsart, ${ }^{55}$ C. Deluca, ${ }^{148}$ S. Demers, ${ }^{175}$ M. Demichev,${ }^{65}$ B. Demirkoz, ${ }^{11}$ J. Deng, ${ }^{163}$ W. Deng,${ }^{24}$ S. P. Denisov, ${ }^{128}$ C. Dennis, ${ }^{118}$ J. E. Derkaoui, ${ }^{135 b}$ F. Derue, ${ }^{78}$ P. Dervan, ${ }^{73}$ K. Desch,${ }^{20}$ P. O. Deviveiros, ${ }^{158}$ A. Dewhurst, ${ }^{129}$ B. DeWilde, ${ }^{148}$ S. Dhaliwal, ${ }^{158}$ R. Dhullipudi, ${ }^{24, m}$ A. Di Ciaccio, ${ }^{133 a, 133 b}$ L. Di Ciaccio, ${ }^{4}$ A. Di Domenico, ${ }^{132 a, 132 b}$ A. Di Girolamo, ${ }^{29}$ B. Di Girolamo, ${ }^{29}$ S. Di Luise,${ }^{134 a, 134 b}$ A. Di Mattia, ${ }^{88}$ R. Di Nardo, ${ }^{133 a, 133 b}$ A. Di Simone, ${ }^{133 a, 133 b}$ R. Di Sipio, ${ }^{19 a, 19 b}$ M. A. Diaz, ${ }^{31 a}$ M. M. Diaz Gomez, ${ }^{49}$ F. Diblen, ${ }^{18 c}$ E. B. Diehl, ${ }^{87}$ H. Dietl, ${ }^{99}$ J. Dietrich, ${ }^{48}$ T. A. Dietzsch, ${ }^{58 a}$ S. Diglio, ${ }^{115}$ K. Dindar Yagci, ${ }^{39}$ J. Dingfelder, ${ }^{20}$ C. Dionisi, ${ }^{132 a, 132 b}$ P. Dita, ${ }^{25 a}$ S. Dita, ${ }^{25 a}$ F. Dittus,${ }^{29}$ F. Djama,${ }^{83}$ R. Djilkibaev, ${ }^{108}$ T. Djobava, ${ }^{51}$ M. A. B. do Vale, ${ }^{23}$ A. Do Valle Wemans, ${ }^{124 a}$ T. K. O. Doan, ${ }^{4}$ M. Dobbs, ${ }^{85}$ R. Dobinson, ${ }^{29, a}$ D. Dobos,${ }^{29}$ E. Dobson, ${ }^{29}$ M. Dobson, ${ }^{163}$ J. Dodd,${ }^{34}$ O. B. Dogan, ${ }^{18 a, a}$ C. Doglioni, ${ }^{118}$ T. Doherty,${ }^{53}$ Y. Doi,${ }^{66}$ J. Dolejsi, ${ }^{126}$ I. Dolenc, ${ }^{74}$ Z. Dolezal, ${ }^{126}$ B. A. Dolgoshein, ${ }^{96}$ T. Dohmae, ${ }^{155}$ M. Donega, ${ }^{120}$ J. Donini, ${ }^{55}$ J. Dopke, ${ }^{174}$ A. Doria, ${ }^{102 a}$ A. Dos Anjos,${ }^{172}$ M. Dosil, ${ }^{11}$ A. Dotti, ${ }^{122 a, 122 b}$ M. T. Dova, ${ }^{70}$ J. D. Dowell, ${ }^{17}$ A. Doxiadis, ${ }^{105}$ A. T. Doyle, ${ }^{53}$ Z. Drasal, ${ }^{126}$ J. Drees, ${ }^{174}$ N. Dressnandt, ${ }^{120}$ H. Drevermann, ${ }^{29}$ C. Driouichi, ${ }^{35}$ M. Dris, ${ }^{9}$ J. G. Drohan, ${ }^{77}$ J. Dubbert, ${ }^{99}$ T. Dubbs, ${ }^{137}$ S. Dube, ${ }^{14}$ E. Duchovni, ${ }^{171}$ G. Duckeck, ${ }^{98}$ A. Dudarev, ${ }^{29}$ F. Dudziak, ${ }^{115}$ M. Dührssen, ${ }^{29}$ I. P. Duerdoth, ${ }^{82}$ L. Duflot,${ }^{115}$ M-A. Dufour, ${ }^{85}$ M. Dunford, ${ }^{29}$ H. Duran Yildiz, ${ }^{3 b}$ A. Dushkin, ${ }^{22}$ R. Duxfield, ${ }^{139}$ M. Dwuznik,${ }^{37}$ F. Dydak, ${ }^{29}$ D. Dzahini, ${ }^{55}$ M. Düren, ${ }^{52}$ W. L. Ebenstein, ${ }^{44}$ J. Ebke, ${ }^{98}$ S. Eckert ${ }^{48}$ S. Eckweiler,${ }^{81}$ K. Edmonds,${ }^{81}$ C. A. Edwards ${ }^{76}$ I. Efthymiopoulos, ${ }^{49}$ K. Egorov, ${ }^{61}$ W. Ehrenfeld ${ }^{41}$ T. Ehrich, ${ }^{99}$ T. Eifert, ${ }^{29}$ G. Eigen,${ }^{13}$ K. Einsweiler, ${ }^{14}$

E. Eisenhandler, ${ }^{75}$ T. Ekelof, ${ }^{166}$ M. El Kacimi,${ }^{4}$ M. Ellert, ${ }^{166}$ S. Elles, ${ }^{4}$ F. Ellinghaus, ${ }^{81}$ K. Ellis, ${ }^{75}$ N. Ellis, ${ }^{29}$ J. Elmsheuser, ${ }^{98}$ M. Elsing ${ }^{29}$ R. Ely,${ }^{14}$ D. Emeliyanov, ${ }^{129}$ R. Engelmann, ${ }^{148}$ A. Engl,${ }^{98}$ B. Epp, ${ }^{62}$ A. Eppig,${ }^{87}$ J. Erdmann, ${ }^{54}$ A. Ereditato, ${ }^{16}$ V. Eremin,,${ }^{97}$ D. Eriksson, ${ }^{146 a}$ I. Ermoline, ${ }^{88}$ J. Ernst, ${ }^{1}$ M. Ernst, ${ }^{24}$ J. Ernwein, ${ }^{136}$ 
D. Errede,${ }^{165}$ S. Errede, ${ }^{165}$ E. Ertel,${ }^{81}$ M. Escalier, ${ }^{115}$ C. Escobar, ${ }^{167}$ X. Espinal Curull, ${ }^{11}$ B. Esposito,${ }^{47}$ F. Etienne,${ }^{83}$ A. I. Etienvre, ${ }^{136}$ E. Etzion, ${ }^{153}$ H. Evans, ${ }^{61}$ V. N. Evdokimov,${ }^{128}$ L. Fabbri, ${ }^{19 a, 19 b}$ C. Fabre, ${ }^{29}$ K. Facius, ${ }^{35}$ R. M. Fakhrutdinov, ${ }^{128}$ S. Falciano, ${ }^{132 a}$ A. C. Falou, ${ }^{115}$ Y. Fang, ${ }^{172}$ M. Fanti, ${ }^{89 a}, 89 b$ A. Farbin, ${ }^{7}$ A. Farilla, ${ }^{134 a}$ J. Farley, ${ }^{148}$ T. Farooque, ${ }^{158}$ S. M. Farrington, ${ }^{118}$ P. Farthouat,${ }^{29}$ D. Fasching, ${ }^{172}$ P. Fassnacht,${ }^{29}$ D. Fassouliotis ${ }^{8}$ B. Fatholahzadeh, ${ }^{158}$ L. Fayard, ${ }^{115}$ S. Fazio, ${ }^{36 a, 36 b}$ R. Febbraro, ${ }^{33}$ P. Federic, ${ }^{144 a}$ O. L. Fedin, ${ }^{121}$ I. Fedorko, ${ }^{29}$ W. Fedorko, ${ }^{29}$ M. Fehling-Kaschek ${ }^{48}$ L. Feligioni, ${ }^{83}$ C. U. Felzmann, ${ }^{86}$ C. Feng, ${ }^{32 \mathrm{~d}}$ E. J. Feng, ${ }^{30}$ A. B. Fenyuk, ${ }^{128}$ J. Ferencei, ${ }^{144 \mathrm{~b}}$ D. Ferguson, ${ }^{172}$ J. Ferland, ${ }^{93}$ B. Fernandes, ${ }^{124 a, n}$ W. Fernando, ${ }^{109}$ S. Ferrag, ${ }^{53}$ J. Ferrando, ${ }^{118}$ V. Ferrara, ${ }^{41}$ A. Ferrari, ${ }^{166}$ P. Ferrari, ${ }^{105}$ R. Ferrari, ${ }^{19 a}$ A. Ferrer,${ }^{167}$ M. L. Ferrer,${ }^{47}$ D. Ferrere, ${ }^{49}$ C. Ferretti, ${ }^{87}$ A. Ferretto Parodi ${ }^{50 a, 50 b}$ F. Ferro, ${ }^{50 a, 50 b}$ M. Fiascaris,${ }^{118}$ F. Fiedler, ${ }^{81}$ A. Filipčič,${ }^{74}$ A. Filippas, ${ }^{9}$ F. Filthaut, ${ }^{104}$ M. Fincke-Keeler, ${ }^{169}$ M. C. N. Fiolhais, ${ }^{124 a, i}$ L. Fiorini, ${ }^{11}$ A. Firan,${ }^{39}$ G. Fischer, ${ }^{41}$ P. Fischer, ${ }^{20}$ M. J. Fisher, ${ }^{109}$

S. M. Fisher, ${ }^{129}$ J. Flammer, ${ }^{29}$ M. Flechl,${ }^{48}$ I. Fleck, ${ }^{141}$ J. Fleckner ${ }^{81}$ P. Fleischmann, ${ }^{173}$ S. Fleischmann, ${ }^{20}$ T. Flick, ${ }^{174}$ L. R. Flores Castillo, ${ }^{172}$ M. J. Flowerdew, ${ }^{99}$ F. Föhlisch, ${ }^{58 a}$ M. Fokitis, ${ }^{9}$ T. Fonseca Martin, ${ }^{16}$ J. Fopma, ${ }^{118}$ D. A. Forbush, ${ }^{138}$ A. Formica, ${ }^{136}$ A. Forti, ${ }^{82}$ D. Fortin, ${ }^{159 a}$ J. M. Foster, ${ }^{82}$ D. Fournier, ${ }^{15}$ A. Foussat, ${ }^{29}$ A. J. Fowler, ${ }^{44}$ K. Fowler, ${ }^{137}$ H. Fox,${ }^{71}$ P. Francavilla, ${ }^{122 a, 122 b}$ S. Franchino, ${ }^{119 a, 119 b}$ D. Francis, ${ }^{29}$ M. Franklin, ${ }^{57}$ S. Franz, ${ }^{29}$ M. Fraternali, ${ }^{119 a, 119 b}$ S. Fratina, ${ }^{120}$ J. Freestone, ${ }^{82}$ S. T. French,${ }^{27}$ R. Froeschl, ${ }^{29}$ D. Froidevaux,${ }^{29}$ J. A. Frost, ${ }^{27}$ C. Fukunaga, ${ }^{156}$ E. Fullana Torregrosa,${ }^{5}$ J. Fuster, ${ }^{167}$ C. Gabaldon, ${ }^{80}$ O. Gabizon, ${ }^{171}$ T. Gadfort, ${ }^{24}$ S. Gadomski, ${ }^{49}$ G. Gagliardi, ${ }^{50 a, 50 b}$ P. Gagnon, ${ }^{61}$ C. Galea, ${ }^{98}$ E. J. Gallas, ${ }^{118}$ M. V. Gallas, ${ }^{29}$ V. Gallo, ${ }^{16}$ B. J. Gallop, ${ }^{129}$ P. Gallus,${ }^{125}$ E. Galyaev, ${ }^{40}$ K. K. Gan, ${ }^{109}$ Y. S. Gao, ${ }^{143, o}$ V. A. Gapienko, ${ }^{128}$ A. Gaponenko, ${ }^{14}$ M. Garcia-Sciveres, ${ }^{14}$ C. García, ${ }^{167}$ J. E. García Navarro, ${ }^{49}$ R. W. Gardner, ${ }^{30}$ N. Garelli, ${ }^{29}$ H. Garitaonandia, ${ }^{105}$ V. Garonne, ${ }^{29}$ J. Garvey, ${ }^{17}$ C. Gatti, ${ }^{47}$ G. Gaudio, ${ }^{19 a}$ O. Gaumer, ${ }^{49}$ V. Gautard ${ }^{136}$ P. Gauzzi, ${ }^{132 a, 132 b}$ I. L. Gavrilenko, ${ }^{94}$ C. Gay, ${ }^{168}$ G. Gaycken,${ }^{20}$ J-C. Gayde ${ }^{29}$ E. N. Gazis, ${ }^{9}$ P. Ge ${ }^{32 \mathrm{~d}}$ C. N.P. Gee, ${ }^{129}$ Ch. Geich-Gimbel,${ }^{20}$ K. Gellerstedt, ${ }^{146 a, 146 b}$ C. Gemme,${ }^{50 a}$ M. H. Genest, ${ }^{98}$ S. Gentile, ${ }^{132 a, 132 b}$ F. Georgatos, ${ }^{9}$ S. George,${ }^{76}$ P. Gerlach, ${ }^{174}$ A. Gershon, ${ }^{153}$ C. Geweniger, ${ }^{58 a}$ H. Ghazlane, ${ }^{135 \mathrm{c}}$ P. Ghez, ${ }^{4}$ N. Ghodbane, ${ }^{33}$ B. Giacobbe, ${ }^{19 \mathrm{a}}$ S. Giagu, ${ }^{132 \mathrm{a}, 132 \mathrm{~b}}$ V. Giakoumopoulou, ${ }^{8}$ V. Giangiobbe, ${ }^{122 a, 122 b}$ F. Gianotti, ${ }^{29}$ B. Gibbard, ${ }^{24}$ A. Gibson, ${ }^{158}$ S. M. Gibson, ${ }^{118}$ G. F. Gieraltowski, ${ }^{5}$ L. M. Gilbert, ${ }^{118}$ M. Gilchriese, ${ }^{14}$ O. Gildemeister, ${ }^{29}$ V. Gilewsky, ${ }^{91}$ D. Gillberg, ${ }^{28}$ A. R. Gillman, ${ }^{129}$ D. M. Gingrich, ${ }^{2, f}$ J. Ginzburg, ${ }^{153}$ N. Giokaris, ${ }^{8}$ M. P. Giordani, ${ }^{164 a, 164 \mathrm{c}}$ R. Giordano, ${ }^{102 a, 102 b}$ F. M. Giorgi, ${ }^{15}$ P. Giovannini, ${ }^{99}$ P. F. Giraud, ${ }^{136}$ P. Girtler, ${ }^{62}$ D. Giugni, ${ }^{89 a}$ P. Giusti, ${ }^{19 a}$ B. K. Gjelsten, ${ }^{117}$ L. K. Gladilin, ${ }^{97}$ C. Glasman, ${ }^{80}$ J Glatzer ${ }^{48}$ A. Glazov,${ }^{41}$ K. W. Glitza, ${ }^{174}$ G. L. Glonti, ${ }^{65}$ K. G. Gnanvo, ${ }^{75}$ J. Godfrey, ${ }^{142}$ J. Godlewski, ${ }^{29}$ M. Goebel, ${ }^{41}$ T. Göpfert, ${ }^{43}$ C. Goeringer,${ }^{81}$ C. Gössling, ${ }^{42}$ T. Göttfert, ${ }^{99}$ V. Goggi, ${ }^{119 a, 119 b, p}$ S. Goldfarb, ${ }^{87}$ D. Goldin, ${ }^{39}$ T. Golling, ${ }^{175}$ N. P. Gollub,${ }^{29}$ S. N. Golovnia, ${ }^{128}$ A. Gomes, ${ }^{124 a, q}$ L. S. Gomez Fajardo, ${ }^{41}$ R. Gonçalo, ${ }^{76}$ L. Gonella, ${ }^{20}$ C. Gong, ${ }^{32 b}$ A. Gonidec,${ }^{29}$ S. Gonzalez, ${ }^{172}$ S. González de la Hoz,,${ }^{167}$ M. L. Gonzalez Silva, ${ }^{26}$ B. Gonzalez-Pineiro, ${ }^{88}$ S. Gonzalez-Sevilla, ${ }^{49}$ J. J. Goodson, ${ }^{148}$ L. Goossens, ${ }^{29}$ P. A. Gorbounov, ${ }^{95}$ H. A. Gordon, ${ }^{24}$ I. Gorelov, ${ }^{103}$ G. Gorfine, ${ }^{174}$ B. Gorini, ${ }^{29}$ E. Gorini, ${ }^{72 a, 72 b}$ A. Gorišek, ${ }^{74}$ E. Gornicki, ${ }^{38}$ S. A. Gorokhov, ${ }^{128}$ B. T. Gorski, ${ }^{29}$ V. N. Goryachev, ${ }^{128}$ B. Gosdzik, ${ }^{41}$ M. Gosselink, ${ }^{105}$ M. I. Gostkin, ${ }^{65}$ M. Gouanère, ${ }^{4}$ I. Gough Eschrich, ${ }^{163}$ M. Gouighri, ${ }^{135 a}$ D. Goujdami, ${ }^{135 a}$ M. P. Goulette, ${ }^{49}$ A. G. Goussiou, ${ }^{138}$ C. Goy, ${ }^{4}$ I. Grabowska-Bold, ${ }^{163, g}$ V. Grabski, ${ }^{176}$ P. Grafström, ${ }^{29}$ C. Grah, ${ }^{174}$ K-J. Grahn, ${ }^{147}$ F. Grancagnolo, ${ }^{72 a}$ S. Grancagnolo, ${ }^{15}$ V. Grassi, ${ }^{148}$ V. Gratchev,${ }^{121}$ N. Grau,${ }^{34}$ H. M. Gray, ${ }^{34, r}$ J. A. Gray, ${ }^{148}$ E. Graziani, ${ }^{134 a}$ O. G. Grebenyuk, ${ }^{121}$ B. Green, ${ }^{76}$ D. Greenfield, ${ }^{129}$ T. Greenshaw, ${ }^{73}$ Z. D. Greenwood, ${ }^{24, m}$ I. M. Gregor, ${ }^{41}$ P. Grenier, ${ }^{143}$ A. Grewal, ${ }^{118}$ E. Griesmayer ${ }^{46}$ J. Griffiths, ${ }^{138}$ N. Grigalashvili, ${ }^{65}$ A. A. Grillo, ${ }^{137}$ K. Grimm, ${ }^{148}$ S. Grinstein, ${ }^{11}$ P. L. Y. Gris, ${ }^{33}$ Y. V. Grishkevich, ${ }^{97}$ J.-F. Grivaz, ${ }^{115}$ L. S. Groer, ${ }^{158}$ J. Grognuz, ${ }^{29}$ M. Groh, ${ }^{99}$ M. Groll, ${ }^{81}$ E. Gross, ${ }^{171}$ J. Grosse-Knetter, ${ }^{54}$ J. Groth-Jensen, ${ }^{79}$ M. Gruwe, ${ }^{29}$ K. Grybel, ${ }^{141}$ V. J. Guarino, ${ }^{5}$ C. Guicheney, ${ }^{33}$ A. Guida, ${ }^{72 a, 72 b}$ T. Guillemin, ${ }^{4}$ H. Guler, ${ }^{85, s}$ J. Gunther, ${ }^{125}$ B. Guo,${ }^{158}$ A. Gupta, ${ }^{30}$ Y. Gusakov, ${ }^{65}$ V. N. Gushchin, ${ }^{128}$ A. Gutierrez, ${ }^{93}$ P. Gutierrez, ${ }^{111}$ N. Guttman, ${ }^{153}$ O. Gutzwiller, ${ }^{172}$ C. Guyot, ${ }^{136}$ C. Gwenlan, ${ }^{118}$ C. B. Gwilliam, ${ }^{73}$ A. Haas, ${ }^{143}$ S. Haas,${ }^{29}$ C. Haber, ${ }^{14}$ G. Haboubi, ${ }^{123}$ R. Hackenburg, ${ }^{24}$ H. K. Hadavand, ${ }^{39}$ D. R. Hadley, ${ }^{17}$ C. Haeberli, ${ }^{16}$ P. Haefner, ${ }^{99}$ R. Härtel, ${ }^{99}$ F. Hahn,${ }^{29}$ S. Haider,${ }^{29}$ Z. Hajduk,${ }^{38}$ H. Hakobyan, ${ }^{176}$ J. Haller, ${ }^{41, t}$ G. D. Hallewell ${ }^{83}$ K. Hamacher, ${ }^{174}$ A. Hamilton, ${ }^{49}$ S. Hamilton, ${ }^{161}$ H. Han, ${ }^{32 a}$ L. Han, ${ }^{32 b}$ K. Hanagaki, ${ }^{116}$ M. Hance, ${ }^{120}$ C. Handel, ${ }^{81}$ P. Hanke, ${ }^{58 a}$ C. J. Hansen, ${ }^{166}$ J. R. Hansen, ${ }^{35}$ J. B. Hansen, ${ }^{35}$ J. D. Hansen, ${ }^{35}$ P. H. Hansen, ${ }^{35}$ T. Hansl-Kozanecka, ${ }^{137}$ P. Hansson, ${ }^{143}$ K. Hara, ${ }^{160}$ G. A. Hare, ${ }^{137}$ T. Harenberg, ${ }^{174}$ R. Harper, ${ }^{139}$ R. D. Harrington, ${ }^{21}$ O. M. Harris, ${ }^{138}$ K Harrison, ${ }^{17}$ J. C. Hart, ${ }^{129}$ J. Hartert,${ }^{48}$ F. Hartjes, ${ }^{105}$ T. Haruyama, ${ }^{66}$ A. Harvey, ${ }^{56}$ S. Hasegawa, ${ }^{101}$ Y. Hasegawa, ${ }^{140}$ K. Hashemi, ${ }^{22}$ S. Hassani, ${ }^{136}$ M. Hatch, ${ }^{29}$ D. Hauff, ${ }^{99}$ S. Haug, ${ }^{16}$ M. Hauschild, ${ }^{29}$ R. Hauser, ${ }^{88}$ M. Havranek, ${ }^{125}$ B. M. Hawes, ${ }^{118}$ C. M. Hawkes,${ }^{17}$ R. J. Hawkings,${ }^{29}$ 
D. Hawkins, ${ }^{163}$ T. Hayakawa, ${ }^{67}$ H. S. Hayward, ${ }^{73}$ S. J. Haywood, ${ }^{129}$ E. Hazen, ${ }^{21}$ M. He, ${ }^{32 d}$ S. J. Head, ${ }^{17}$ V. Hedberg ${ }^{79}$ L. Heelan, ${ }^{28}$ S. Heim,${ }^{88}$ B. Heinemann, ${ }^{14}$ F. E. W. Heinemann, ${ }^{118}$ S. Heisterkamp,${ }^{35}$ L. Helary, ${ }^{4}$ M. Heldmann, ${ }^{48}$ M. Heller, ${ }^{115}$ S. Hellman, ${ }^{146 a, 146 b}$ C. Helsens,${ }^{11}$ T. Hemperek, ${ }^{20}$ R. C. W. Henderson, ${ }^{71}$ P. J. Hendriks, ${ }^{105}$ M. Henke, ${ }^{58 a}$ A. Henrichs, ${ }^{54}$ A. M. Henriques Correia,${ }^{29}$ S. Henrot-Versille, ${ }^{115}$ F. Henry-Couannier, ${ }^{83}$ C. Hensel, ${ }^{54}$ T. Henß, ${ }^{174}$ Y. Hernández Jiménez, ${ }^{167}$ A. D. Hershenhorn, ${ }^{152}$ G. Herten, ${ }^{48}$ R. Hertenberger, ${ }^{98}$ L. Hervas, ${ }^{29}$ N. P. Hessey, ${ }^{105}$ A. Hidvegi, ${ }^{146 a}$ E. Higón-Rodriguez, ${ }^{167}$ D. Hill, ${ }^{5, a}$ J. C. Hill, ${ }^{27}$ N. Hill, ${ }^{5}$ K. H. Hiller, ${ }^{41}$ S. Hillert, ${ }^{20}$ S. J. Hillier, ${ }^{17}$ I. Hinchliffe, ${ }^{14}$ D. Hindson, ${ }^{118}$ E. Hines, ${ }^{120}$ M. Hirose, ${ }^{116}$ F. Hirsch, ${ }^{42}$ D. Hirschbuehl, ${ }^{174}$ J. Hobbs, ${ }^{148}$ N. Hod, ${ }^{153}$ M. C. Hodgkinson, ${ }^{139}$ P. Hodgson, ${ }^{139}$ A. Hoecker, ${ }^{29}$ M. R. Hoeferkamp, ${ }^{103}$ J. Hoffman,${ }^{39}$ D. Hoffmann, ${ }^{83}$ M. Hohlfeld, ${ }^{81}$ M. Holder, ${ }^{141}$ T. I. Hollins, ${ }^{17}$ G. Hollyman, ${ }^{76}$ A. Holmes ${ }^{118}$ S. O. Holmgren, ${ }^{146 a}$ T. Holy, ${ }^{127}$ J. L. Holzbauer, ${ }^{88}$ R. J. Homer, ${ }^{17}$ Y. Homma ${ }^{67}$ T. Horazdovsky, ${ }^{127}$ C. Horn, ${ }^{143}$ S. Horner, ${ }^{48}$ S. Horvat, ${ }^{99}$ J-Y. Hostachy, ${ }^{55}$ T. Hott,${ }^{99}$ S. Hou, ${ }^{151}$ M. A. Houlden ${ }^{73}$ A. Hoummada, ${ }^{135 a}$ D. F. Howell, ${ }^{118}$ J. Hrivnac, ${ }^{115}$ I. Hruska, ${ }^{125}$ T. Hryn'ova, ${ }^{4}$ P. J. Hsu, ${ }^{175}$ S.-C. Hsu, ${ }^{14}$ G. S. Huang, ${ }^{111}$ Z. Hubacek, ${ }^{127}$ F. Hubaut, ${ }^{83}$ F. Huegging, ${ }^{20}$ T. B. Huffman, ${ }^{118}$ E. W. Hughes,${ }^{34}$ G. Hughes,${ }^{71}$ R. E. Hughes-Jones,${ }^{82}$ M. Huhtinen, ${ }^{29}$ P. Hurst, ${ }^{57}$ M. Hurwitz, ${ }^{30}$ U. Husemann,${ }^{41}$ N. Huseynov, ${ }^{10}$ J. Huston, ${ }^{88}$ J. Huth,${ }^{57}$ G. Iacobucci, ${ }^{102 a}$ G. Iakovidis, ${ }^{9}$ M. Ibbotson, ${ }^{82}$ I. Ibragimov, ${ }^{141}$ R. Ichimiya,${ }^{67}$ L. Iconomidou-Fayard, ${ }^{115}$ J. Idarraga,${ }^{159 b} \mathrm{M}$. Idzik, ${ }^{37}$ P. Iengo, ${ }^{4}$ O. Igonkina, ${ }^{105}$ Y. Ikegami ${ }^{66}$ M. Ikeno, ${ }^{66}$ Y. Ilchenko, ${ }^{39}$ D. Iliadis, ${ }^{154}$ D. Imbault, ${ }^{78}$ M. Imhaeuser,${ }^{174}$ M. Imori, ${ }^{155}$ T. Ince, ${ }^{20} \mathrm{~J}$. Inigo-Golfin, ${ }^{29} \mathrm{P}$. Ioannou, ${ }^{8} \mathrm{M}$. Iodice, ${ }^{134 a} \mathrm{G}$. Ionescu, ${ }^{4} \mathrm{~A}$. Irles Quiles, ${ }^{167} \mathrm{~K}$. Ishii, ${ }^{66} \mathrm{~A}$. Ishikawa, ${ }^{67}$ M. Ishino, ${ }^{66}$ R. Ishmukhametov, ${ }^{39}$ T. Isobe, ${ }^{155}$ V. Issakov, ${ }^{175, a} \mathrm{C}$. Issever, ${ }^{18}$ S. Istin, ${ }^{18 a}$ Y. Itoh, ${ }^{101} \mathrm{~A}$. V. Ivashin, ${ }^{128}$ W. Iwanski, ${ }^{38}$ H. Iwasaki, ${ }^{66}$ J. M. Izen,${ }^{40}$ V. Izzo, ${ }^{102 a}$ B. Jackson, ${ }^{120}$ J. N. Jackson, ${ }^{73}$ P. Jackson, ${ }^{143}$ M. R. Jaekel, ${ }^{29}$ M. Jahoda, ${ }^{125}$ V. Jain ${ }^{61}$ K. Jakobs,${ }^{48}$ S. Jakobsen, ${ }^{35}$ J. Jakubek, ${ }^{127}$ D. K. Jana, ${ }^{111}$ E. Jankowski, ${ }^{158}$ E. Jansen, ${ }^{77}$

A. Jantsch, ${ }^{99}$ M. Janus, ${ }^{20}$ R. C. Jared,,${ }^{172}$ G. Jarlskog, ${ }^{79}$ L. Jeanty, ${ }^{57}$ K. Jelen, ${ }^{37}$ I. Jen-La Plante, ${ }^{30}$ P. Jenni, ${ }^{29}$ A. Jeremie, ${ }^{4}$ P. Jež,${ }^{35}$ S. Jézéquel, ${ }^{4} \mathrm{H}$. Ji,,${ }^{172}$ W. Ji, ${ }^{79}$ J. Jia, ${ }^{148}$ Y. Jiang, ${ }^{32 b}$ M. Jimenez Belenguer, ${ }^{29}$ G. Jin, ${ }^{32 b}$ S. Jin, ${ }^{32 a}$ O. Jinnouchi, ${ }^{157}$ D. Joffe, ${ }^{39}$ L. G. Johansen, ${ }^{13}$ M. Johansen, ${ }^{146 a, 146 b}$ K. E. Johansson, ${ }^{146 a}$ P. Johansson, ${ }^{139}$ S. Johnert, ${ }^{41}$ K. A. Johns, ${ }^{6}$ K. Jon-And,,${ }^{146 a, 146 b}$ G. Jones, ${ }^{82}$ M. Jones, ${ }^{118}$ R. W. L. Jones, ${ }^{71}$ T. W. Jones, ${ }^{77}$ T. J. Jones,${ }^{73}$ O. Jonsson, ${ }^{29}$ K. K. Joo, ${ }^{158, u}$ D. Joos, ${ }^{48}$ C. Joram,${ }^{29}$ P. M. Jorge, ${ }^{124 a, d}$ S. Jorgensen, ${ }^{11}$ J. Joseph, ${ }^{14}$ V. Juranek, ${ }^{125}$ P. Jussel, ${ }^{62}$ V. V. Kabachenko, ${ }^{128}$ S. Kabana, ${ }^{16}$ M. Kaci, ${ }^{167}$ A. Kaczmarska, ${ }^{38}$ P. Kadlecik, ${ }^{35}$ M. Kado, ${ }^{115}$ H. Kagan, ${ }^{109}$ M. Kagan,${ }^{57}$ S. Kaiser, ${ }^{99}$ E. Kajomovitz, ${ }^{152}$ S. Kalinin,${ }^{174}$ L. V. Kalinovskaya, ${ }^{65}$ S. Kama, ${ }^{39}$ N. Kanaya, ${ }^{155}$ M. Kaneda, ${ }^{155}$ V. A. Kantserov,${ }^{96}$ J. Kanzaki, ${ }^{66}$ B. Kaplan, ${ }^{175}$ A. Kapliy, ${ }^{30}$ J. Kaplon, ${ }^{29}$ D. Kar, ${ }^{43}$ M. Karagounis, ${ }^{20}$ M. Karagoz, ${ }^{118}$ M. Karnevskiy, ${ }^{41}$ K. Karr, ${ }^{5}$ V. Kartvelishvili, ${ }^{71}$ A. N. Karyukhin, ${ }^{128}$ L. Kashif, ${ }^{57}$ A. Kasmi ${ }^{39}$ R. D. Kass, ${ }^{109}$ A. Kastanas, ${ }^{13}$ M. Kastoryano, ${ }^{175}$ M. Kataoka,,${ }^{4}$ Y. Kataoka, ${ }^{155}$ E. Katsoufis, ${ }^{9}$ J. Katzy, ${ }^{41}$ V. Kaushik, ${ }^{6}$ K. Kawagoe, ${ }^{67}$ T. Kawamoto, ${ }^{155}$ G. Kawamura,${ }^{81}$ M. S. Kayl,${ }^{105}$ F. Kayumov, ${ }^{94}$ V. A. Kazanin, ${ }^{107}$ M. Y. Kazarinov, ${ }^{65}$ S. I. Kazi, ${ }^{86}$ J. R. Keates, ${ }^{82}$ R. Keeler, ${ }^{169}$ P. T. Keener ${ }^{120}$ R. Kehoe, ${ }^{39}$ M. Keil,${ }^{54}$ G. D. Kekelidze, ${ }^{65}$ M. Kelly, ${ }^{82}$ J. Kennedy,${ }^{98}$ C. J. Kenney, ${ }^{143}$ M. Kenyon, ${ }^{53}$ O. Kepka, ${ }^{125}$ N. Kerschen, ${ }^{29}$ B. P. Kerševan, ${ }^{74}$ S. Kersten, ${ }^{174}$ K. Kessoku, ${ }^{155}$ C. Ketterer, ${ }^{48}$ M. Khakzad,${ }^{28}$ F. Khalil-zada, ${ }^{10}$ H. Khandanyan, ${ }^{165}$ A. Khanov, ${ }^{112}$ D. Kharchenko, ${ }^{65}$ A. Khodinov, ${ }^{148}$ A. G. Kholodenko, ${ }^{128}$ A. Khomich, ${ }^{58 a}$ G. Khoriauli, ${ }^{20}$ N. Khovanskiy, ${ }^{65}$ V. Khovanskiy, ${ }^{95}$ E. Khramov ${ }^{65}$ J. Khubua, ${ }^{51}$ G. Kilvington, ${ }^{76}$ H. Kim, ${ }^{7}$ M. S. Kim, ${ }^{2}$ P. C. Kim, ${ }^{143}$ S. H. Kim, ${ }^{160}$ N. Kimura, ${ }^{170}$ O. Kind, ${ }^{15}$ P. Kind, ${ }^{174}$ B. T. King, ${ }^{73}$ M. King, ${ }^{67}$ J. Kirk, ${ }^{129}$ G. P. Kirsch, ${ }^{118}$ L. E. Kirsch, ${ }^{22}$ A. E. Kiryunin, ${ }^{99}$ D. Kisielewska, ${ }^{37}$ B. Kisielewski, ${ }^{38}$ T. Kittelmann, ${ }^{123}$ A. M. Kiver, ${ }^{128}$ H. Kiyamura, ${ }^{67}$

E. Kladiva, ${ }^{144 \mathrm{~b}}$ J. Klaiber-Lodewigs, ${ }^{42}$ M. Klein, ${ }^{73}$ U. Klein, ${ }^{73}$ K. Kleinknecht,${ }^{81}$ M. Klemetti ${ }^{85}$ A. Klier, ${ }^{171}$ A. Klimentov, ${ }^{24}$ R. Klingenberg, ${ }^{42}$ E. B. Klinkby, ${ }^{44}$ T. Klioutchnikova, ${ }^{29}$ P. F. Klok, ${ }^{104}$ S. Klous, ${ }^{105}$ E.-E. Kluge,${ }^{58 a}$ T. Kluge, ${ }^{73}$ P. Kluit, ${ }^{105}$ S. Kluth, ${ }^{99}$ N. S. Knecht, ${ }^{158}$ E. Kneringer, ${ }^{62}$ J. Knobloch, ${ }^{29}$ B. R. Ko, ${ }^{44}$ T. Kobayashi, ${ }^{155}$ M. Kobel,${ }^{43}$ B. Koblitz,${ }^{29}$ M. Kocian, ${ }^{143}$ A. Kocnar,${ }^{13}$ P. Kodys, ${ }^{126}$ K. Köneke, ${ }^{29}$ A. C. König, ${ }^{104}$ S. Koenig, ${ }^{81}$ S. König, ${ }^{48}$ L. Köpke, ${ }^{81}$ F. Koetsveld, ${ }^{104}$ P. Koevesarki ${ }^{20}$ T. Koffas, ${ }^{29}$ E. Koffeman, ${ }^{105}$ F. Kohn, ${ }^{54}$ Z. Kohout, ${ }^{127}$ T. Kohriki, ${ }^{66}$ T. Koi, ${ }^{143}$ T. Kokott, ${ }^{20}$ G. M. Kolachev, ${ }^{107}$ H. Kolanoski, ${ }^{15}$ V. Kolesnikov, ${ }^{65}$ I. Koletsou, ${ }^{4}$ J. Koll, ${ }^{88}$ D. Kollar, ${ }^{29}$ M. Kollefrath, ${ }^{48}$ S. Kolos, ${ }^{163, v}$ S. D. Kolya,${ }^{82}$ A. A. Komar, ${ }^{94}$ J. R. Komaragiri, ${ }^{142}$ T. Kondo, ${ }^{66}$ T. Kono, ${ }^{41, t}$ A. I. Kononov, ${ }^{48}$ R. Konoplich, ${ }^{108}$ S. P. Konovalov, ${ }^{94}$ N. Konstantinidis, ${ }^{77}$ A. Kootz, ${ }^{174}$ S. Koperny, ${ }^{37}$ S. V. Kopikov, ${ }^{128}$ K. Korcyl,${ }^{38}$ K. Kordas, ${ }^{154}$ V. Koreshev, ${ }^{128}$ A. Korn,${ }^{14}$ A. Korol, ${ }^{107}$ I. Korolkov, ${ }^{11}$

E. V. Korolkova, ${ }^{139}$ V. A. Korotkov, ${ }^{128}$ O. Kortner, ${ }^{99}$ P. Kostka ${ }^{41}$ V. V. Kostyukhin, ${ }^{20}$ M. J. Kotamäki, ${ }^{29}$ S. Kotov, ${ }^{99}$ V. M. Kotov, ${ }^{65}$ K. Y. Kotov ${ }^{107}$ C. Kourkoumelis, ${ }^{8}$ A. Koutsman, ${ }^{105}$ R. Kowalewski, ${ }^{169}$ H. Kowalski, ${ }^{41}$

T. Z. Kowalski, ${ }^{37}$ W. Kozanecki, ${ }^{136}$ A. S. Kozhin, ${ }^{128}$ V. Kral, ${ }^{127}$ V. A. Kramarenko, ${ }^{97}$ G. Kramberger, ${ }^{74}$ O. Krasel, ${ }^{42}$ M. W. Krasny, ${ }^{78}$ A. Krasznahorkay, ${ }^{108}$ J. Kraus, ${ }^{88}$ A. Kreisel, ${ }^{153}$ F. Krejci, ${ }^{127}$ J. Kretzschmar, ${ }^{73}$ N. Krieger, ${ }^{54}$ 
P. Krieger, ${ }^{158}$ G. Krobath, ${ }^{98}$ K. Kroeninger, ${ }^{54}$ H. Kroha, ${ }^{99}$ J. Kroll, ${ }^{120}$ J. Kroseberg, ${ }^{20}$ J. Krstic, ${ }^{12 a}$ U. Kruchonak, ${ }^{65}$ H. Krüger, ${ }^{20}$ Z. V. Krumshteyn, ${ }^{65}$ A. Kruth, ${ }^{20}$ T. Kubota, ${ }^{155}$ S. Kuehn, ${ }^{48}$ A. Kugel, ${ }^{58 \mathrm{c}}$ T. Kuhl, ${ }^{174}$ D. Kuhn, ${ }^{62}$ V. Kukhtin, ${ }^{65}$ Y. Kulchitsky, ${ }^{90}$ S. Kuleshov, ${ }^{31 b}$ C. Kummer,${ }^{98}$ M. Kuna,${ }^{83}$ N. Kundu, ${ }^{118}$ J. Kunkle, ${ }^{120}$ A. Kupco, ${ }^{125}$ H. Kurashige, ${ }^{67}$ M. Kurata, ${ }^{160}$ L. L. Kurchaninov, ${ }^{159 a}$ Y. A. Kurochkin, ${ }^{90}$ V. Kus, ${ }^{125}$ W. Kuykendall, ${ }^{138}$ M. Kuze,${ }^{157}$ P. Kuzhir, ${ }^{91}$ O. Kvasnicka, ${ }^{125}$ R. Kwee, ${ }^{15}$ A. La Rosa, ${ }^{29}$ L. La Rotonda, ${ }^{36 a, 36 b}$ L. Labarga, ${ }^{80}$ J. Labbe, ${ }^{4}$ C. Lacasta, ${ }^{167}$ F. Lacava, ${ }^{132 a, 132 b}$ H. Lacker, ${ }^{15}$ D. Lacour, ${ }^{78}$ V. R. Lacuesta, ${ }^{167}$ E. Ladygin, ${ }^{65}$ R. Lafaye, ${ }^{4}$ B. Laforge, ${ }^{78}$ T. Lagouri, ${ }^{80}$ S. Lai ${ }^{48}$ M. Lamanna, ${ }^{29}$ M. Lambacher ${ }^{98}$ C. L. Lampen, ${ }^{6}$ W. Lampl,${ }^{6}$ E. Lancon, ${ }^{136}$ U. Landgraf, ${ }^{48}$

M. P. J. Landon, ${ }^{75}$ H. Landsman, ${ }^{152}$ J. L. Lane, ${ }^{82}$ C. Lange, ${ }^{41}$ A. J. Lankford, ${ }^{163}$ F. Lanni, ${ }^{24}$ K. Lantzsch, ${ }^{29}$ A. Lanza, ${ }^{119 a}$ V. V. Lapin, ${ }^{128, a}$ S. Laplace, ${ }^{4}$ C. Lapoire, ${ }^{83}$ J. F. Laporte, ${ }^{136}$ T. Lari, ${ }^{89 a}$ A. V. Larionov, ${ }^{128}$ A. Larner, ${ }^{118}$ C. Lasseur, ${ }^{29}$ M. Lassnig, ${ }^{29}$ W. Lau, ${ }^{118}$ P. Laurelli, ${ }^{47}$ A. Lavorato, ${ }^{118}$ W. Lavrijsen, ${ }^{14}$ P. Laycock,${ }^{73}$ A. B. Lazarev, ${ }^{65}$ A. Lazzaro, ${ }^{89 a, 89 b}$ O. Le Dortz, ${ }^{78}$ E. Le Guirriec,${ }^{83}$ C. Le Maner, ${ }^{158}$ E. Le Menedeu, ${ }^{136}$ M. Le Vine, ${ }^{24}$ M. Leahu, ${ }^{29}$

A. Lebedev, ${ }^{64}$ C. Lebel, ${ }^{93}$ M. Lechowski, ${ }^{115}$ T. LeCompte, ${ }^{5}$ F. Ledroit-Guillon, ${ }^{55}$ H. Lee, ${ }^{105}$ J. S. H. Lee,${ }^{150}$

S. C. Lee, ${ }^{151}$ M. Lefebvre, ${ }^{169}$ M. Legendre, ${ }^{136}$ A. Leger, ${ }^{49}$ B. C. LeGeyt, ${ }^{120}$ F. Legger ${ }^{98}$ C. Leggett, ${ }^{14}$ M. Lehmacher, ${ }^{20}$ G. Lehmann Miotto, ${ }^{29}$ M. Lehto, ${ }^{139}$ X. Lei, ${ }^{6}$ R. Leitner, ${ }^{126}$ D. Lellouch, ${ }^{171}$ J. Lellouch, ${ }^{78}$ M. Leltchouk, ${ }^{34}$ V. Lendermann, ${ }^{58 a}$ K. J. C. Leney, ${ }^{73}$ T. Lenz, ${ }^{174}$ G. Lenzen, ${ }^{174}$ B. Lenzi, ${ }^{136}$ K. Leonhardt, ${ }^{43}$ J. Lepidis, ${ }^{174}$ C. Leroy, ${ }^{93}$ J-R. Lessard, ${ }^{169}$ J. Lesser, ${ }^{146 a}$ C. G. Lester,${ }^{27}$ A. Leung Fook Cheong, ${ }^{172}$ J. Levêque, ${ }^{83}$ D. Levin, ${ }^{87}$ L. J. Levinson, ${ }^{171}$ M. S. Levitski, ${ }^{128}$ M. Lewandowska, ${ }^{21}$ M. Leyton, ${ }^{15} \mathrm{H} . \mathrm{Li},{ }^{172} \mathrm{X}$. Li, ${ }^{87} \mathrm{Z}$. Liang, ${ }^{39}$ Z. Liang, ${ }^{118, w}$ B. Liberti, ${ }^{133 a}$ P. Lichard, ${ }^{29}$ M. Lichtnecker, ${ }^{98}$ K. Lie, ${ }^{165}$ W. Liebig, ${ }^{173}$ R. Lifshitz, ${ }^{152}$ J. N. Lilley, ${ }^{17}$ H. Lim, ${ }^{5}$ A. Limosani, ${ }^{86}$ M. Limper, ${ }^{63}$ S. C. Lin ${ }^{151}$ F. Linde, ${ }^{105}$ J. T. Linnemann ${ }^{88}$ E. Lipeles, ${ }^{120}$ L. Lipinsky, ${ }^{125}$ A. Lipniacka, ${ }^{13}$ T. M. Liss, ${ }^{165}$ D. Lissauer, ${ }^{24}$ A. Lister ${ }^{49}$ A. M. Litke, ${ }^{137}$ C. Liu, ${ }^{28}$ D. Liu, ${ }^{151, x}$ H. Liu, ${ }^{87}$ J. B. Liu, ${ }^{87}$ M. Liu, ${ }^{32 b}$ S. Liu, ${ }^{2}$ T. Liu, ${ }^{39}$ Y. Liu, ${ }^{32 b}$ M. Livan, ${ }^{19 a, 119 b}$ S. S. A. Livermore, ${ }^{18}$ A. Lleres, ${ }^{55}$ S. L. Lloyd, ${ }^{75}$

E. Lobodzinska, ${ }^{41}$ P. Loch, ${ }^{6}$ W. S. Lockman, ${ }^{137}$ S. Lockwitz, ${ }^{175}$ T. Loddenkoetter, ${ }^{20}$ F. K. Loebinger, ${ }^{82}$ A. Loginov, ${ }^{175}$ C. W. Loh, ${ }^{168}$ T. Lohse, ${ }^{15}$ K. Lohwasser, ${ }^{48}$ M. Lokajicek, ${ }^{125}$ J. Loken, ${ }^{118}$ R. E. Long, ${ }^{71}$ L. Lopes, ${ }^{124 a, d}$ D. Lopez Mateos, ${ }^{34, \mathrm{r}}$ M. Losada, ${ }^{162}$ P. Loscutoff, ${ }^{14}$ M. J. Losty, ${ }^{159 a}$ X. Lou ${ }^{40}$ A. Lounis, ${ }^{115}$ K. F. Loureiro, ${ }^{162}$ L. Lovas, ${ }^{144 a}$ J. Love, ${ }^{21}$ P. A. Love, ${ }^{71}$ A. J. Lowe, ${ }^{143}$ F. Lu, ${ }^{32 a}$ J. Lu, ${ }^{2}$ L. Lu, ${ }^{39}$ H. J. Lubatti, ${ }^{138}$ C. Luci, ${ }^{132 a, 132 b}$ A. Lucotte,${ }^{55}$ A. Ludwig, ${ }^{43}$ D. Ludwig, ${ }^{41}$ I. Ludwig, ${ }^{48}$ J. Ludwig, ${ }^{48}$ F. Luehring, ${ }^{61}$ G. Luijckx, ${ }^{105}$ D. Lumb, ${ }^{48}$ L. Luminari, ${ }^{132 \mathrm{a}}$ E. Lund, ${ }^{117}$ B. Lund-Jensen, ${ }^{147}$ B. Lundberg, ${ }^{79}$ J. Lundberg, ${ }^{29}$ J. Lundquist, ${ }^{35}$ A. Lupi, ${ }^{122 a, 122 b}$ G. Lutz, ${ }^{99}$ D. Lynn, ${ }^{24}$ J. Lynn, ${ }^{118}$ J. Lys, ${ }^{14}$ E. Lytken, ${ }^{79}$ H. Ma,${ }^{24}$ L. L. Ma,${ }^{172}$ M. Maßen, ${ }^{48}$ J. A. Macana Goia, ${ }^{93}$ G. Maccarrone, ${ }^{47}$ A. Macchiolo, ${ }^{99}$ B. Maček, ${ }^{74}$ J. Machado Miguens, ${ }^{124 a, d}$ D. Macina, ${ }^{49}$ R. Mackeprang,,${ }^{35}$

D. MacQueen, ${ }^{2}$ R. J. Madaras, ${ }^{14}$ W. F. Mader,${ }^{43}$ R. Maenner,${ }^{58 c}$ T. Maeno,${ }^{24}$ P. Mättig, ${ }^{174}$ S. Mättig,${ }^{41}$ P. J. Magalhaes Martins, ${ }^{124 a, \mathrm{i}}$ L. Magnoni ${ }^{29}$ E. Magradze,${ }^{51}$ C. A. Magrath,${ }^{104}$ Y. Mahalalel,,${ }^{153}$ K. Mahboubi, ${ }^{48}$ A. Mahmood, ${ }^{1}$ G. Mahout, ${ }^{17}$ C. Maiani, ${ }^{132 a, 132 b}$ C. Maidantchik,${ }^{23}$ A. Maio, ${ }^{124 a, q}$ S. Majewski, ${ }^{24}$ Y. Makida, ${ }^{66}$ M. Makouski, ${ }^{128}$ N. Makovec, ${ }^{115}$ P. Mal, ${ }^{6}$ Pa. Malecki, ${ }^{38}$ P. Malecki, ${ }^{38}$ V. P. Maleev, ${ }^{121}$ F. Malek,,${ }^{55}$ U. Mallik,${ }^{63}$

D. Malon, ${ }^{5}$ S. Maltezos, ${ }^{9}$ V. Malyshev, ${ }^{107}$ S. Malyukov, ${ }^{65}$ M. Mambelli, ${ }^{30}$ R. Mameghani, ${ }^{98}$ J. Mamuzic,${ }^{41}$

A. Manabe, ${ }^{66}$ A. Manara, ${ }^{61}$ L. Mandelli, ${ }^{89 a}$ I. Mandić, ${ }^{74}$ R. Mandrysch,,${ }^{15}$ J. Maneira, ${ }^{124 a}$ P. S. Mangeard,${ }^{88}$ M. Mangin-Brinet, ${ }^{49}$ I. D. Manjavidze ${ }^{65}$ A. Mann,${ }^{54}$ W. A. Mann, ${ }^{161}$ P. M. Manning, ${ }^{137}$ A. Manousakis-Katsikakis, ${ }^{8}$

B. Mansoulie, ${ }^{136}$ A. Manz,${ }^{99}$ A. Mapelli, ${ }^{29}$ L. Mapelli, ${ }^{29}$ L. March, ${ }^{80}$ J. F. Marchand, ${ }^{4}$ F. Marchese, ${ }^{133 a, 133 b}$

M. Marchesotti, ${ }^{29}$ G. Marchiori, ${ }^{78}$ M. Marcisovsky, ${ }^{125}$ A. Marin, ${ }^{21, a}$ C. P. Marino, ${ }^{61}$ F. Marroquim, ${ }^{23}$ R. Marshall, ${ }^{82}$ Z. Marshall, ${ }^{34, \mathrm{r}}$ F. K. Martens, ${ }^{158}$ S. Marti-Garcia, ${ }^{167}$ A. J. Martin, ${ }^{75}$ A. J. Martin, ${ }^{175}$ B. Martin, ${ }^{29}$ B. Martin, ${ }^{88}$

F. F. Martin, ${ }^{120}$ J. P. Martin, ${ }^{93} \mathrm{Ph}$. Martin, ${ }^{55}$ T. A. Martin, ${ }^{17}$ B. Martin dit Latour, ${ }^{49}$ M. Martinez,${ }^{11}$

V. Martinez Outschoorn, ${ }^{57}$ A. Martini, ${ }^{47}$ A. C. Martyniuk, ${ }^{82}$ F. Marzano, ${ }^{132 a}$ A. Marzin, ${ }^{136}$ L. Masetti, ${ }^{81}$ T. Mashimo, ${ }^{155}$ R. Mashinistov, ${ }^{94}$ J. Masik, ${ }^{82}$ A. L. Maslennikov, ${ }^{107}$ M. Maß, ${ }^{42}$ I. Massa, ${ }^{19 a, 19 b}$ G. Massaro, ${ }^{105}$

N. Massol, ${ }^{4}$ A. Mastroberardino, ${ }^{36 a, 36 b}$ T. Masubuchi, ${ }^{155}$ M. Mathes, ${ }^{20}$ P. Matricon, ${ }^{115}$ H. Matsumoto,,${ }^{155}$ H. Matsunaga ${ }^{155}$ T. Matsushita, ${ }^{67}$ C. Mattravers, ${ }^{118, y}$ J. M. Maugain, ${ }^{29}$ S. J. Maxfield, ${ }^{73}$ E. N. May, ${ }^{5}$ J. K. Mayer, ${ }^{158}$ A. Mayne, ${ }^{199}$ R. Mazini, ${ }^{151}$ M. Mazur, ${ }^{20}$ M. Mazzanti, ${ }^{89 a}$ E. Mazzoni, ${ }^{122 a, 122 b}$ J. Mc Donald, ${ }^{85}$ S. P. Mc Kee, ${ }^{87}$ A. McCarn, ${ }^{165}$ R. L. McCarthy, ${ }^{148}$ T. G. McCarthy, ${ }^{28}$ N. A. McCubbin,${ }^{129}$ K. W. McFarlane,${ }^{56}$ S. McGarvie, ${ }^{76}$ H. McGlone, ${ }^{53}$ G. Mchedlidze, ${ }^{51}$ R. A. McLaren, ${ }^{29}$ S. J. McMahon, ${ }^{129}$ T. R. McMahon, ${ }^{76}$ T. J. McMahon, ${ }^{17}$ R. A. McPherson, ${ }^{169,1}$ A. Meade, ${ }^{84}$ J. Mechnich, ${ }^{105}$ M. Mechtel,,${ }^{174}$ M. Medinnis, ${ }^{41}$ R. Meera-Lebbai, ${ }^{111}$

T. Meguro, ${ }^{116}$ R. Mehdiyev, ${ }^{93}$ S. Mehlhase, ${ }^{41}$ A. Mehta, ${ }^{73}$ K. Meier, ${ }^{58 a}$ J. Meinhardt, ${ }^{48}$ B. Meirose,${ }^{48}$ C. Melachrinos,${ }^{30}$ B. R. Mellado Garcia,${ }^{172}$ L. Mendoza Navas, ${ }^{162}$ Z. Meng, ${ }^{151, \mathrm{x}}$ A. Mengarelli, ${ }^{19 \mathrm{a}, 19 \mathrm{~b}}$ S. Menke, ${ }^{99}$ C. Menot ${ }^{29}$ E. Meoni, ${ }^{11}$ D. Merkl, ${ }^{98}$ P. Mermod, ${ }^{118}$ L. Merola, ${ }^{102 a, 102 b}$ C. Meroni, ${ }^{89 a}$ F. S. Merritt, ${ }^{30}$ 
A. M. Messina, ${ }^{29}$ I. Messmer, ${ }^{48}$ J. Metcalfe, ${ }^{103}$ A. S. Mete, ${ }^{64}$ S. Meuser, ${ }^{20}$ C. Meyer, ${ }^{81}$ J-P. Meyer, ${ }^{136}$ J. Meyer,${ }^{173}$ J. Meyer, ${ }^{54}$ T. C. Meyer, ${ }^{29}$ W. T. Meyer, ${ }^{64}$ J. Miao, ${ }^{32 d}$ S. Michal, ${ }^{29}$ L. Micu, ${ }^{25 a}$ R. P. Middleton, ${ }^{129}$ P. Miele,${ }^{29}$ S. Migas, ${ }^{73}$ A. Migliaccio, ${ }^{102 a, 102 b}$ L. Mijović, ${ }^{41}$ G. Mikenberg, ${ }^{171}$ M. Mikestikova, ${ }^{125}$ B. Mikulec ${ }^{49}$ M. Mikuž ${ }^{74}$ D. W. Miller, ${ }^{143}$ R. J. Miller, ${ }^{88}$ W. J. Mills, ${ }^{168}$ C. Mills,${ }^{57}$ A. Milov, ${ }^{171}$ D. A. Milstead, ${ }^{146 a, 146 b}$ D. Milstein, ${ }^{171}$ S. Mima, ${ }^{110}$ A. A. Minaenko, ${ }^{128}$ M. Miñano, ${ }^{167}$ I. A. Minashvili, ${ }^{65}$ A. I. Mincer, ${ }^{108}$ B. Mindur,${ }^{37}$ M. Mineev, ${ }^{65}$ Y. Ming, ${ }^{130}$ L. M. Mir, ${ }^{11}$ G. Mirabelli, ${ }^{132 a}$ L. Miralles Verge,${ }^{11}$ S. Misawa,${ }^{24}$ S. Miscetti, ${ }^{47}$ A. Misiejuk, ${ }^{76}$ A. Mitra, ${ }^{118}$ J. Mitrevski, ${ }^{137}$ G. Y. Mitrofanov, ${ }^{128}$ V. A. Mitsou, ${ }^{167}$ S. Mitsui, ${ }^{66}$ P. S. Miyagawa, ${ }^{82}$ K. Miyazaki, ${ }^{67}$

J. U. Mjörnmark, ${ }^{79}$ D. Mladenov, ${ }^{22}$ T. Moa, ${ }^{146 a, 146 b}$ M. Moch, ${ }^{132 a, 132 b}$ P. Mockett, ${ }^{138}$ S. Moed ${ }^{57}$ V. Moeller ${ }^{27}$ K. Mönig, ${ }^{41}$ N. Möser, ${ }^{20}$ B. Mohn, ${ }^{13}$ W. Mohr, ${ }^{48}$ S. Mohrdieck-Möck, ${ }^{99}$ A. M. Moisseev, ${ }^{128, a}$ R. Moles-Valls, ${ }^{167}$ J. Molina-Perez ${ }^{29}$ L. Moneta, ${ }^{49}$ J. Monk,${ }^{77}$ E. Monnier, ${ }^{83}$ S. Montesano, ${ }^{89 a, 89 b}$ F. Monticelli, ${ }^{70}$ R. W. Moore, ${ }^{2}$ G. F. Moorhead, ${ }^{86}$ C. Mora Herrera, ${ }^{49}$ A. Moraes,${ }^{53}$ A. Morais, ${ }^{124 a, d}$ J. Morel,${ }^{54}$ G. Morello, ${ }^{36 a, 36 b}$ D. Moreno, ${ }^{81}$ M. Moreno Llácer, ${ }^{167}$ P. Morettini, ${ }^{50 a}$ D. Morgan,${ }^{139}$ M. Morii, ${ }^{57}$ J. Morin,${ }^{75}$ Y. Morita ${ }^{66}$ A. K. Morley, ${ }^{29}$ G. Mornacchi, ${ }^{29}$ M-C. Morone, ${ }^{49}$ S. V. Morozov, ${ }^{96}$ J. D. Morris, ${ }^{75}$ H. G. Moser,${ }^{99}$ M. Mosidze,${ }^{51}$ J. Moss, ${ }^{109}$ A. Moszczynski, ${ }^{38}$ R. Mount, ${ }^{143}$ E. Mountricha, ${ }^{9}$ S. V. Mouraviev, ${ }^{94}$ T. H. Moye,${ }^{17}$ E. J. W. Moyse, ${ }^{84}$ M. Mudrinic,${ }^{12 b}$ F. Mueller, ${ }^{58 a}$ J. Mueller, ${ }^{123}$ K. Mueller ${ }^{20}$ T. A. Müller, ${ }^{98}$ D. Muenstermann, ${ }^{42}$ A. Muijs, ${ }^{105}$ A. Muir, ${ }^{168}$ A. Munar, ${ }^{120}$ Y. Munwes, ${ }^{153}$ K. Murakami, ${ }^{66}$ R. Murillo Garcia, ${ }^{163}$ W. J. Murray, ${ }^{129}$ I. Mussche, ${ }^{105}$ E. Musto, ${ }^{102 a, 102 b}$ A. G. Myagkov, ${ }^{128}$ M. Myska, ${ }^{125}$ J. Nadal, ${ }^{11}$ K. Nagai, ${ }^{160}$ K. Nagano, ${ }^{66}$ Y. Nagasaka, ${ }^{60}$ A. M. Nairz, ${ }^{29}$ D. Naito, ${ }^{110}$ K. Nakamura, ${ }^{155}$ I. Nakano, ${ }^{110}$ G. Nanava, ${ }^{20}$ A. Napier, ${ }^{161}$ M. Nash,${ }^{77, y}$ I. Nasteva, ${ }^{82}$ N. R. Nation, ${ }^{21}$ T. Nattermann, ${ }^{20}$ T. Naumann, ${ }^{41}$ F. Nauyock, ${ }^{82}$ G. Navarro, ${ }^{162}$ S. K. Nderitu, ${ }^{85}$ H. A. Neal, ${ }^{87}$ E. Nebot,${ }^{80}$ P. Nechaeva, ${ }^{94}$ A. Negri, ${ }^{119 a, 119 b}$ G. Negri, ${ }^{29}$ S. Negroni, ${ }^{34}$ A. Nelson, ${ }^{64}$ S. Nelson, ${ }^{143}$ T. K. Nelson, ${ }^{143}$ S. Nemecek, ${ }^{125}$ P. Nemethy, ${ }^{108}$ A. A. Nepomuceno, ${ }^{23}$ M. Nessi, ${ }^{29}$ S. Y. Nesterov, ${ }^{121}$ M. S. Neubauer, ${ }^{165}$ L. Neukermans, ${ }^{4}$ A. Neusiedl,${ }^{81}$ R. M. Neves,${ }^{108}$ P. Nevski,${ }^{24}$ F. M. Newcomer, ${ }^{120}$ C. Nicholson, ${ }^{53}$ R. B. Nickerson, ${ }^{118}$ R. Nicolaidou, ${ }^{136}$ L. Nicolas,${ }^{139}$ G. Nicoletti, ${ }^{47}$ B. Nicquevert, ${ }^{29}$ F. Niedercorn, ${ }^{115}$ J. Nielsen, ${ }^{137}$ T. Niinikoski, ${ }^{29}$ A. Nikiforov, ${ }^{15}$ V. Nikolaenko, ${ }^{128}$ K. Nikolaev, ${ }^{65}$ I. Nikolic-Audit, ${ }^{78}$ K. Nikolopoulos,${ }^{24}$ H. Nilsen, ${ }^{48}$ P. Nilsson, ${ }^{7}$ Y. Ninomiya, ${ }^{155}$ A. Nisati, ${ }^{132 a}$ T. Nishiyama,${ }^{67}$ R. Nisius, ${ }^{99}$ L. Nodulman,,${ }^{5}$ M. Nomachi,,${ }^{16}$ I. Nomidis, ${ }^{154}$ H. Nomoto, ${ }^{155}$ M. Nordberg, ${ }^{29}$ B. Nordkvist, ${ }^{146 a, 146 b}$ O. Norniella Francisco, ${ }^{11}$ P. R. Norton, ${ }^{129}$ D. Notz, ${ }^{41}$ J. Novakova, ${ }^{126}$ M. Nozaki, ${ }^{66}$ M. Nožička, ${ }^{41}$ I. M. Nugent, ${ }^{159 a}$ A.-E. Nuncio-Quiroz, ${ }^{20}$

G. Nunes Hanninger, ${ }^{20}$ T. Nunnemann, ${ }^{98}$ E. Nurse, ${ }^{77}$ T. Nyman, ${ }^{29}$ S. W. O'Neale, ${ }^{17, a}$ D. C. O’ Neil, ${ }^{142}$ V. O'Shea, ${ }^{53}$ F. G. Oakham, ${ }^{28, f}$ H. Oberlack, ${ }^{99}$ J. Ocariz, ${ }^{78}$ A. Ochi, ${ }^{67}$ S. Oda, ${ }^{155}$ S. Odaka, ${ }^{66}$ J. Odier, ${ }^{83}$ G. A. Odino, ${ }^{50,50 b}$ H. Ogren, ${ }^{61}$ A. Oh, ${ }^{82}$ S. H. Oh, ${ }^{44}$ C. C. Ohm, ${ }^{146 a, 146 b}$ T. Ohshima, ${ }^{101}$ H. Ohshita, ${ }^{140}$ T. K. Ohska, ${ }^{66}$ T. Ohsugi, ${ }^{59}$ S. Okada, ${ }^{67}$ H. Okawa, ${ }^{163}$ Y. Okumura, ${ }^{101}$ T. Okuyama, ${ }^{155}$ M. Olcese, ${ }^{50 a}$ A. G. Olchevski, ${ }^{65}$ M. Oliveira, ${ }^{124 a, i}$ D. Oliveira Damazio, ${ }^{24}$ C. Oliver, ${ }^{80}$ J. Oliver, ${ }^{57}$ E. Oliver Garcia,${ }^{167}$ D. Olivito, ${ }^{120}$ A. Olszewski, ${ }^{38}$ J. Olszowska, ${ }^{38}$ C. Omachi, ${ }^{67, z}$ A. Onofre, ${ }^{124 a, a a}$ P. U.E. Onyisi,${ }^{30}$ C. J. Oram, ${ }^{159 a}$ G. Ordonez, ${ }^{104}$ M. J. Oreglia, ${ }^{30}$ F. Orellana, ${ }^{49}$ Y. Oren, ${ }^{153}$ D. Orestano, ${ }^{134 a, 134 b}$ I. Orlov, ${ }^{107}$ C. Oropeza Barrera, ${ }^{53}$ R. S. Orr, ${ }^{158}$ E. O. Ortega, ${ }^{130}$ B. Osculati, ${ }^{50 a, 50 b}$ R. Ospanov, ${ }^{120}$ C. Osuna, ${ }^{11}$ G. Otero y Garzon, ${ }^{26}$ J. P. Ottersbach, ${ }^{105}$ B. Ottewell, ${ }^{118}$ M. Ouchrif, ${ }^{135 b}$ F. Ould-Saada, ${ }^{17}$ A. Ouraou, ${ }^{136}$ Q. Ouyang, ${ }^{32 \mathrm{a}}$ M. Owen, ${ }^{82}$ S. Owen, ${ }^{139}$ A Oyarzun, ${ }^{31 \mathrm{~b}}$ O. K. Øye, ${ }^{13}$ V. E. Ozcan, ${ }^{77}$ K. Ozone,${ }^{66}$ N. Ozturk, ${ }^{7}$ A. Pacheco Pages,${ }^{11}$ C. Padilla Aranda,${ }^{11}$ E. Paganis, ${ }^{139}$ F. Paige, ${ }^{24}$ K. Pajchel,${ }^{17}$ S. Palestini, ${ }^{29}$ J. Palla, ${ }^{29}$ D. Pallin, ${ }^{33}$ A. Palma, ${ }^{14 a, d}$ J. D. Palmer, ${ }^{17}$ M. J. Palmer, ${ }^{27}$ Y. B. Pan, ${ }^{172}$ E. Panagiotopoulou, ${ }^{9}$ B. Panes, ${ }^{31 \mathrm{a}}$ N. Panikashvili, ${ }^{87}$ V. N. Panin, ${ }^{107}$ S. Panitkin, ${ }^{24}$ D. Pantea, ${ }^{25 a}$ M. Panuskova, ${ }^{125}$ V. Paolone,${ }^{123}$ A. Paoloni, ${ }^{133 a, 133 b}$ Th. D. Papadopoulou, ${ }^{9}$ A. Paramonov, ${ }^{5}$ S. J. Park, ${ }^{54}$ W. Park,${ }^{24, b b}$ M. A. Parker,${ }^{27}$ S. I. Parker, ${ }^{14}$ F. Parodi, ${ }^{50 a, 50 b}$ J. A. Parsons,${ }^{34}$ U. Parzefall, ${ }^{48}$ E. Pasqualucci, ${ }^{132 a}$ A. Passeri, ${ }^{134 a}$ F. Pastore, ${ }^{134 a, 134 b}$ Fr. Pastore, ${ }^{29}$ G. Pásztor, ${ }^{49, c c}$ S. Pataraia, ${ }^{172}$ N. Patel, ${ }^{150}$ J. R. Pater, ${ }^{82}$ S. Patricelli, ${ }^{102 a, 102 b}$ T. Pauly, ${ }^{29}$ L. S. Peak, ${ }^{150}$ M. Pecsy, ${ }^{144 a}$ M. I. Pedraza Morales, ${ }^{172}$ S. J. M. Peeters, ${ }^{105}$ S. V. Peleganchuk, ${ }^{107}$ H. Peng, ${ }^{172}$ R. Pengo, ${ }^{29}$ A. Penson, ${ }^{34}$ J. Penwell, ${ }^{61}$ M. Perantoni ${ }^{23}$ K. Perez,${ }^{34, \mathrm{r}}$ E. Perez Codina,${ }^{11}$ M. T. Pérez García-Estañ, ${ }^{167}$ V. Perez Reale, ${ }^{34}$ I. Peric,${ }^{20}$ L. Perini, ${ }^{89 a, 89 b}$ H. Pernegger, ${ }^{29}$ R. Perrino, ${ }^{72 a}$ P. Perrodo, ${ }^{4}$ S. Persembe, ${ }^{3 a}$ P. Perus,${ }^{115}$

V. D. Peshekhonov, ${ }^{65}$ E. Petereit, ${ }^{5}$ O. Peters, ${ }^{105}$ B. A. Petersen, ${ }^{29}$ J. Petersen, ${ }^{29}$ T. C. Petersen, ${ }^{35}$ E. Petit, ${ }^{83}$ C. Petridou, ${ }^{154}$ E. Petrolo, ${ }^{132 \mathrm{a}}$ F. Petrucci, ${ }^{134 \mathrm{a}, 134 \mathrm{~b}}$ D Petschull, ${ }^{41}$ M. Petteni, ${ }^{142}$ R. Pezoa, ${ }^{31 \mathrm{~b}}$ B. Pfeifer, ${ }^{48}$ A. Phan, ${ }^{86}$ A. W. Phillips,${ }^{27}$ G. Piacquadio, ${ }^{29}$ E. Piccaro,${ }^{75}$ M. Piccinini, ${ }^{19 a, 19 b}$ A. Pickford ${ }^{53}$ R. Piegaia, ${ }^{26}$ J. E. Pilcher, ${ }^{30}$

A. D. Pilkington, ${ }^{82}$ J. Pina, ${ }^{124 a, q}$ M. Pinamonti, ${ }^{164 a, 164 c}$ J. L. Pinfold, ${ }^{2}$ J. Ping, ${ }^{32 \mathrm{c}}$ B. Pinto, ${ }^{124 a, d}$ O. Pirotte, ${ }^{29}$ C. Pizio, ${ }^{89 a, 89 b}$ R. Placakyte, ${ }^{41}$ M. Plamondon, ${ }^{169}$ W. G. Plano,${ }^{82}$ M.-A. Pleier, ${ }^{24}$ A. V. Pleskach, ${ }^{128}$ A. Poblaguev,${ }^{175}$ S. Poddar ${ }^{58 \mathrm{a}}$ F. Podlyski, ${ }^{33}$ P. Poffenberger,${ }^{169}$ L. Poggioli, ${ }^{115}$ T. Poghosyan, ${ }^{20}$ M. Pohl,${ }^{49}$ F. Polci, ${ }^{55}$ G. Polesello, ${ }^{119 a}$ 
A. Policicchio, ${ }^{138}$ A. Polini, ${ }^{19 a}$ J. Poll, ${ }^{75}$ V. Polychronakos, ${ }^{24}$ D. M. Pomarede, ${ }^{136}$ D. Pomeroy, ${ }^{22}$ K. Pommès, ${ }^{29}$ P. Ponsot, ${ }^{136}$ L. Pontecorvo, ${ }^{132 a}$ B. G. Pope ${ }^{88}$ G. A. Popeneciu, ${ }^{25 a}$ R. Popescu, ${ }^{24}$ D. S. Popovic, ${ }^{12 a}$ A. Poppleton, ${ }^{29}$ J. Popule, ${ }^{125}$ X. Portell Bueso, ${ }^{48}$ R. Porter,${ }^{163}$ C. Posch,${ }^{21}$ G. E. Pospelov, ${ }^{99}$ S. Pospisil, ${ }^{127}$ M. Potekhin, ${ }^{24}$ I. N. Potrap, ${ }^{99}$ C. J. Potter, ${ }^{149}$ C. T. Potter ${ }^{85}$ K. P. Potter,${ }^{82}$ G. Poulard,${ }^{29}$ J. Poveda,${ }^{172}$ R. Prabhu,${ }^{20}$ P. Pralavorio, ${ }^{83}$

S. Prasad, ${ }^{57}$ M. Prata, ${ }^{119 a, 119 b}$ R. Pravahan, ${ }^{7}$ K. Pretzl,,${ }^{16}$ L. Pribyl,${ }^{29}$ D. Price,${ }^{61}$ L. E. Price, ${ }^{5}$ M. J. Price,${ }^{29}$ P. M. Prichard ${ }^{73}$ D. Prieur, ${ }^{123}$ M. Primavera, ${ }^{72 a}$ K. Prokofiev ${ }^{29}$ F. Prokoshin, ${ }^{31 b}$ S. Protopopescu, ${ }^{24}$ J. Proudfoot,${ }^{5}$ X. Prudent, ${ }^{43}$ H. Przysiezniak, ${ }^{4}$ S. Psoroulas, ${ }^{20}$ E. Ptacek, ${ }^{114}$ C. Puigdengoles, ${ }^{11}$ J. Purdham, ${ }^{87}$ M. Purohit, ${ }^{24, d d}$ P. Puzo, ${ }^{115}$ Y. Pylypchenko, ${ }^{17}$ M. Qi,${ }^{32 \mathrm{c}}$ J. Qian, ${ }^{87}$ W. Qian, ${ }^{129}$ Z. Qian, ${ }^{83}$ Z. Qin, ${ }^{41}$ D. Qing, ${ }^{151, e e}$ A. Quadt, ${ }^{54}$ D. R. Quarrie, ${ }^{14}$ W. B. Quayle, ${ }^{172}$ F. Quinonez,${ }^{31 a}$ M. Raas, ${ }^{104}$ V. Radeka, ${ }^{24}$ V. Radescu, ${ }^{58 b}$ B. Radics,${ }^{20}$ T. Rador, ${ }^{18 a}$ F. Ragusa, ${ }^{89 a, 89 b}$ G. Rahal, ${ }^{177}$ A. M. Rahimi, ${ }^{109}$ D. Rahm, ${ }^{24}$ C. Raine, ${ }^{53, a}$ B. Raith, ${ }^{20}$ S. Rajagopalan, ${ }^{24}$ S. Rajek, ${ }^{42}$ M. Rammensee, ${ }^{48}$ M. Rammes, ${ }^{141}$ M. Ramstedt,${ }^{146 a, 146 b}$ P. N. Ratoff, ${ }^{71}$ F. Rauscher,${ }^{98}$ E. Rauter, ${ }^{99}$ M. Raymond, ${ }^{29}$ A. L. Read, ${ }^{117}$ D. M. Rebuzzi, ${ }^{119 a, 119 b}$ A. Redelbach, ${ }^{173}$ G. Redlinger, ${ }^{24}$ R. Reece, ${ }^{120}$ K. Reeves,${ }^{40}$ A. Reichold,${ }^{105}$ E. Reinherz-Aronis, ${ }^{153}$ A Reinsch, ${ }^{114}$ I. Reisinger, ${ }^{42}$ D. Reljic, ${ }^{12 a}$ C. Rembser, ${ }^{29}$ Z. L. Ren,${ }^{151}$ P. Renkel, ${ }^{39}$ B. Rensch, ${ }^{35}$ S. Rescia, ${ }^{24}$ M. Rescigno, ${ }^{132 a}$ S. Resconi, ${ }^{89 a}$ B. Resende, ${ }^{136}$ P. Reznicek, ${ }^{126}$ R. Rezvani, ${ }^{158}$ A. Richards,${ }^{77}$ R. A. Richards,${ }^{88}$ R. Richter, ${ }^{99}$ E. Richter-Was,${ }^{38, f f}$ M. Ridel,${ }^{78}$ S. Rieke,${ }^{81}$ M. Rijpstra, ${ }^{105}$ M. Rijssenbeek, ${ }^{148}$ A. Rimoldi, ${ }^{119 a, 119 b}$ L. Rinaldi, ${ }^{19 a}$ R. R. Rios, ${ }^{39}$ I. Riu, ${ }^{11}$ G. Rivoltella, ${ }^{89 a, 89 b}$ F. Rizatdinova, ${ }^{112}$ E. Rizvi, ${ }^{75}$ D. A. Roa Romero, ${ }^{162}$ S. H. Robertson, ${ }^{85,1}$ A. Robichaud-Veronneau, ${ }^{49}$ D. Robinson, ${ }^{27}$ JEM Robinson, ${ }^{77}$ M. Robinson, ${ }^{114}$ A. Robson, ${ }^{53}$ J. G. Rocha de Lima, ${ }^{106}$ C. Roda, ${ }^{122 a, 122 b}$ D. Roda Dos Santos, ${ }^{29}$ S. Rodier, ${ }^{80}$

D. Rodriguez, ${ }^{162}$ Y. Rodriguez Garcia, ${ }^{15}$ S. Roe, ${ }^{29}$ O. Røhne, ${ }^{117}$ V. Rojo, ${ }^{1}$ S. Rolli, ${ }^{161}$ A. Romaniouk, ${ }^{96}$

V. M. Romanov, ${ }^{65}$ G. Romeo, ${ }^{26}$ D. Romero Maltrana,${ }^{31 a}$ L. Roos,${ }^{78}$ E. Ros, ${ }^{167}$ S. Rosati, ${ }^{138}$ G. A. Rosenbaum, ${ }^{158}$ E. I. Rosenberg, ${ }^{64}$ P. L. Rosendahl, ${ }^{13}$ L. Rosselet, ${ }^{49}$ V. Rossetti, ${ }^{11}$ L. P. Rossi, ${ }^{50 a}$ L. Rossi,,${ }^{89,89 b}$ M. Rotaru, ${ }^{25 a}$ J. Rothberg, ${ }^{138}$ I. Rottländer, ${ }^{20}$ D. Rousseau, ${ }^{115}$ C. R. Royon, ${ }^{136}$ A. Rozanov, ${ }^{83}$ Y. Rozen, ${ }^{152}$ X. Ruan, ${ }^{115}$ B. Ruckert, ${ }^{98}$ N. Ruckstuhl, ${ }^{105}$ V. I. Rud,${ }^{97}$ G. Rudolph, ${ }^{62}$ F. Rühr, ${ }^{6}$ F. Ruggieri, ${ }^{134 a}$ A. Ruiz-Martinez, ${ }^{64}$ E. Rulikowska-Zarebska, ${ }^{37}$ V. Rumiantsev, ${ }^{91, a}$ L. Rumyantsev,${ }^{65} \mathrm{~K}$. Runge, ${ }^{48}$ O. Runolfsson, ${ }^{20} \mathrm{Z}$. Rurikova, ${ }^{48}$ N. A. Rusakovich, ${ }^{65}$ D. R. Rust, ${ }^{61}$ J. P. Rutherfoord ${ }^{6}$ C. Ruwiedel,${ }^{20}$ P. Ruzicka, ${ }^{125}$ Y. F. Ryabov, ${ }^{121}$ V. Ryadovikov, ${ }^{128}$ P. Ryan, ${ }^{88}$ G. Rybkin, ${ }^{115}$ S. Rzaeva, ${ }^{10}$ A. F. Saavedra, ${ }^{150}$ H. F-W. Sadrozinski, ${ }^{137}$ R. Sadykov, ${ }^{65}$ F. Safai Tehrani, ${ }^{132 a, 132 b}$ H. Sakamoto, ${ }^{155}$ P. Sala, ${ }^{89 a}$ G. Salamanna, ${ }^{105}$ A. Salamon, ${ }^{133 a}$ M. Saleem, ${ }^{111}$ D. Salihagic, ${ }^{99}$ A. Salnikov, ${ }^{143}$ J. Salt, ${ }^{167}$ O. Saltó Bauza, ${ }^{11}$ B. M. Salvachua Ferrando, ${ }^{5}$ D. Salvatore, ${ }^{36 a, 36 b}$ F. Salvatore, ${ }^{149}$ A. Salvucci, ${ }^{47}$ A. Salzburger, ${ }^{29}$ D. Sampsonidis, ${ }^{154}$ B. H. Samset, ${ }^{117}$ H. Sandaker, ${ }^{13}$ H. G. Sander, ${ }^{81}$ M. P. Sanders,${ }^{98}$ M. Sandhoff, ${ }^{174}$ P. Sandhu, ${ }^{158}$ T. Sandoval, ${ }^{27}$ R. Sandstroem, ${ }^{105}$ S. Sandvoss, ${ }^{174}$ D. P. C. Sankey, ${ }^{129}$ B. Sanny, ${ }^{174}$

A. Sansoni ${ }^{47}$ C. Santamarina Rios,${ }^{85}$ C. Santoni, ${ }^{33}$ R. Santonico, ${ }^{133 a, 133 b}$ H. Santos, ${ }^{124 a}$ J. G. Saraiva, ${ }^{124 a, q}$ T. Sarangi, ${ }^{172}$ E. Sarkisyan-Grinbaum, ${ }^{7}$ F. Sarri, ${ }^{122 a, 122 b}$ G. Sartisohn, ${ }^{174}$ O. Sasaki, ${ }^{66}$ T. Sasaki, ${ }^{66}$ N. Sasao, ${ }^{68}$ I. Satsounkevitch, ${ }^{90}$ G. Sauvage,${ }^{4}$ P. Savard,${ }^{158, f}$ A. Y. Savine,${ }^{6}$ V. Savinov, ${ }^{123}$ P. Savva, ${ }^{9}$ L. Sawyer,${ }^{24, m}$ D. H. Saxon, ${ }^{53}$ L. P. Says, ${ }^{33}$ C. Sbarra, ${ }^{19 a, 19 b}$ A. Sbrizzi,,${ }^{19 a, 19 b}$ O. Scallon, ${ }^{93}$ D. A. Scannicchio, ${ }^{29}$ J. Schaarschmidt ${ }^{43}$ P. Schacht, ${ }^{99}$ U. Schäfer, ${ }^{81}$ S. Schaetzel, ${ }^{58 b}$ A. C. Schaffer, ${ }^{115}$ D. Schaile, ${ }^{98}$ M. Schaller ${ }^{29}$ R. D. Schamberger ${ }^{148}$ A. G. Schamov, ${ }^{107}$ V. Scharf, ${ }^{58 a}$ V. A. Schegelsky, ${ }^{121}$ D. Scheirich,${ }^{87}$ M. Schernau, ${ }^{163}$ M. I. Scherzer, ${ }^{14}$ C. Schiavi, ${ }^{50 a, 50 b}$ J. Schieck, ${ }^{99}$ M. Schioppa, ${ }^{36 a, 36 b}$ S. Schlenker, ${ }^{29}$ J. L. Schlereth, ${ }^{5}$ E. Schmidt, ${ }^{48}$ M. P. Schmidt, ${ }^{175, a}$ K. Schmieden, ${ }^{20}$ C. Schmitt, ${ }^{81}$ M. Schmitz, ${ }^{20}$ R. C. Scholte, ${ }^{105}$ A. Schöning, ${ }^{58 b}$ M. Schott, ${ }^{29}$ D. Schouten, ${ }^{142}$ J. Schovancova, ${ }^{125}$ M. Schram, ${ }^{85}$ A. Schreiner, ${ }^{63}$ C. Schroeder, ${ }^{81}$ N. Schroer, ${ }^{58 c}$ M. Schroers, ${ }^{174}$ D. Schroff,${ }^{48}$ S. Schuh, ${ }^{29}$ G. Schuler, ${ }^{29}$ J. Schultes, ${ }^{174}$ H.-C. Schultz-Coulon, ${ }^{58 a}$ J. W. Schumacher, ${ }^{43}$ M. Schumacher, ${ }^{48}$ B. A. Schumm, ${ }^{137} \mathrm{Ph}$. Schune, ${ }^{136}$ C. Schwanenberger ${ }^{82}$ A. Schwartzman, ${ }^{143}$ D. Schweiger, ${ }^{29} \mathrm{Ph}$. Schwemling, ${ }^{78}$ R. Schwienhorst, ${ }^{88}$ R. Schwierz, ${ }^{43}$ J. Schwindling, ${ }^{136}$ W. G. Scott, ${ }^{129}$ J. Searcy, ${ }^{114}$ E. Sedykh, ${ }^{121}$ E. Segura, ${ }^{11}$ S. C. Seidel, ${ }^{103}$ A. Seiden, ${ }^{137}$ F. Seifert, ${ }^{43}$ J. M. Seixas, ${ }^{23}$ G. Sekhniaidze, ${ }^{102 a}$ D. M. Seliverstov, ${ }^{121}$ B. Sellden, ${ }^{146 a}$ G. Sellers, ${ }^{73}$ M. Seman, ${ }^{144 \mathrm{~b}}$ N. Semprini-Cesari, ${ }^{19 a, 19 b}$ C. Serfon, ${ }^{98}$ L. Serin, ${ }^{115}$ R. Seuster, ${ }^{99}$ H. Severini, ${ }^{111}$ M. E. Sevior, ${ }^{86}$ A. Sfyrla, ${ }^{29}$ E. Shabalina, ${ }^{54}$ M. Shamim,,${ }^{114}$ L. Y. Shan, ${ }^{32 a}$ J. T. Shank, ${ }^{21}$ Q. T. Shao, ${ }^{86}$ M. Shapiro, ${ }^{14}$ P. B. Shatalov, ${ }^{95}$ L. Shaver, ${ }^{6}$ C. Shaw,${ }^{53}$ K. Shaw, ${ }^{139}$ D. Sherman, ${ }^{29}$ P. Sherwood, ${ }^{77}$ A. Shibata, ${ }^{108}$ P. Shield,${ }^{118}$ S. Shimizu, ${ }^{29}$ M. Shimojima, ${ }^{100}$ T. Shin,${ }^{56}$ A. Shmeleva, ${ }^{94}$ M. J. Shochet,${ }^{30}$ M. A. Shupe, ${ }^{6}$ P. Sicho, ${ }^{125}$ A. Sidoti, ${ }^{15}$ A. Siebel,,${ }^{174}$ M. Siebel,${ }^{29}$ F Siegert,${ }^{77}$ J. Siegrist,${ }^{14}$ Dj. Sijacki, ${ }^{12 a}$ O. Silbert, ${ }^{171}$ J. Silva, ${ }^{124 a, g g}$ Y. Silver,${ }^{153}$ D. Silverstein, ${ }^{143}$ S. B. Silverstein, ${ }^{146 a}$ V. Simak, ${ }^{127}$ Lj. Simic, ${ }^{12 a}$ S. Simion, ${ }^{115}$ B. Simmons, ${ }^{77}$ M. Simonyan, ${ }^{35}$ P. Sinervo, ${ }^{158}$ N. B. Sinev, ${ }^{114}$ V. Sipica, ${ }^{141}$ G. Siragusa, ${ }^{81}$ A. N. Sisakyan, ${ }^{65}$ S. Yu. Sivoklokov, ${ }^{97}$ J. Sjölin, ${ }^{146 a, 146 b}$ T. B. Sjursen, ${ }^{13}$ L. A. Skinnari, ${ }^{14}$ K. Skovpen, ${ }^{107}$ P. Skubic, ${ }^{111}$ N. Skvorodnev,${ }^{22}$ M. Slater, ${ }^{17}$ T. Slavicek, ${ }^{127}$ 
K. Sliwa, ${ }^{161}$ T. J. Sloan, ${ }^{71}$ J. Sloper, ${ }^{29}$ V. Smakhtin, ${ }^{171}$ S. Yu. Smirnov, ${ }^{96}$ Y. Smirnov, ${ }^{24}$ L. N. Smirnova, ${ }^{97}$ O. Smirnova, ${ }^{79}$ B. C. Smith, ${ }^{57}$ D. Smith, ${ }^{143}$ K. M. Smith, ${ }^{53}$ M. Smizanska, ${ }^{71}$ K. Smolek, ${ }^{127}$ A. A. Snesarev, ${ }^{94}$ S. W. Snow, ${ }^{82}$ J. Snow, ${ }^{111}$ J. Snuverink, ${ }^{105}$ S. Snyder, ${ }^{24}$ M. Soares,${ }^{124 a}$ R. Sobie, ${ }^{169,1}$ J. Sodomka, ${ }^{127}$ A. Soffer, ${ }^{153}$ C. A. Solans, ${ }^{167}$ M. Solar, ${ }^{127}$ J. Solc, ${ }^{127}$ E. Solfaroli Camillocci, ${ }^{132 a, 132 b}$ A. A. Solodkov, ${ }^{128}$ O. V. Solovyanov, ${ }^{128}$ R. Soluk, ${ }^{2}$ J. Sondericker, ${ }^{24}$ N. Soni,${ }^{2}$ V. Sopko, ${ }^{127}$ B. Sopko, ${ }^{127}$ M. Sorbi,${ }^{89 a, 89 b}$ M. Sosebee, ${ }^{7}$ A. Soukharev, ${ }^{107}$

S. Spagnolo, ${ }^{72 a, 72 b}$ F. Spanò, ${ }^{34}$ P. Speckmayer, ${ }^{29}$ E. Spencer, ${ }^{137}$ R. Spighi, ${ }^{19 a}$ G. Spigo, ${ }^{29}$ F. Spila, ${ }^{132 a, 132 b}$ E. Spiriti, ${ }^{134 a}$ R. Spiwoks, ${ }^{29}$ L. Spogli, ${ }^{134 a, 134 b}$ M. Spousta, ${ }^{126}$ T. Spreitzer, ${ }^{158}$ B. Spurlock, ${ }^{7}$ R. D. St. Denis, ${ }^{53}$

T. Stahl, ${ }^{141}$ J. Stahlman, ${ }^{120}$ R. Stamen, ${ }^{58 a}$ S. N. Stancu, ${ }^{163}$ E. Stanecka, ${ }^{29}$ R. W. Stanek, ${ }^{5}$ C. Stanescu, ${ }^{134 a}$ S. Stapnes, ${ }^{117}$ E. A. Starchenko, ${ }^{128}$ J. Stark, ${ }^{55}$ P. Staroba, ${ }^{125}$ P. Starovoitov, ${ }^{91}$ J. Stastny,${ }^{125}$ A. Staude, ${ }^{98}$ P. Stavina, ${ }^{144 a}$ G. Stavropoulos, ${ }^{14}$ G. Steele,${ }^{53}$ E. Stefanidis,${ }^{77}$ P. Steinbach, ${ }^{43}$ P. Steinberg, ${ }^{24}$ I. Stekl,${ }^{127}$ B. Stelzer, ${ }^{142}$ H. J. Stelzer, ${ }^{41}$

O. Stelzer-Chilton, ${ }^{159 a}$ H. Stenzel,,${ }^{52}$ K. Stevenson, ${ }^{75}$ G. A. Stewart, ${ }^{53}$ W. Stiller, ${ }^{99}$ T. Stockmanns,,${ }^{20}$

M. C. Stockton, ${ }^{29}$ M. Stodulski, ${ }^{38}$ K. Stoerig, ${ }^{48}$ G. Stoicea ${ }^{25 a}$ S. Stonjek, ${ }^{99}$ P. Strachota, ${ }^{126}$ A. R. Stradling, ${ }^{7}$

A. Straessner, ${ }^{43}$ J. Strandberg, ${ }^{87}$ S. Strandberg, ${ }^{14}$ A. Strandlie, ${ }^{117}$ M. Strang, ${ }^{109}$ M. Strauss, ${ }^{111}$ P. Strizenec,${ }^{144 b}$ R. Ströhmer, ${ }^{173}$ D. M. Strom, ${ }^{114}$ J. A. Strong, ${ }^{76, a}$ R. Stroynowski, ${ }^{39}$ J. Strube, ${ }^{129}$ B. Stugu, ${ }^{13}$ I. Stumer, ${ }^{24, a}$ P. Sturm, ${ }^{174}$ D. A. Soh, ${ }^{151, w}$ D. Su, ${ }^{143}$ S. Subramania, ${ }^{61}$ Y. Sugaya, ${ }^{116}$ T. Sugimoto, ${ }^{101}$ C. Suhr, ${ }^{106}$ K. Suita, ${ }^{67}$ M. Suk, ${ }^{126}$ V. V. Sulin, ${ }^{94}$ S. Sultansoy, ${ }^{3 \mathrm{~d}}$ T. Sumida, ${ }^{29}$ X. H. Sun, ${ }^{32 \mathrm{~d}}$ J. E. Sundermann, ${ }^{48}$ K. Suruliz, ${ }^{164 a, 164 \mathrm{~b}}$ S. Sushkov, ${ }^{11}$ G. Susinno, ${ }^{36 a, 36 b}$ M. R. Sutton, ${ }^{139}$ Y. Suzuki, ${ }^{66}$ Yu. M. Sviridov, ${ }^{128}$ S. Swedish, ${ }^{168}$ I. Sykora, ${ }^{144 a}$ T. Sykora, ${ }^{126}$ R. R. Szczygiel, ${ }^{38}$ B. Szeless, ${ }^{29}$ T. Szymocha, ${ }^{38}$ J. Sánchez, ${ }^{167}$ D. Ta, ${ }^{20}$ S. Taboada Gameiro, ${ }^{29}$ K. Tackmann ${ }^{29}$ A. Taffard, ${ }^{163}$ R. Tafirout, ${ }^{159 a}$ A. Taga,${ }^{117}$ Y. Takahashi,,${ }^{101}$ H. Takai, ${ }^{24}$ R. Takashima,${ }^{69}$ H. Takeda, ${ }^{67}$ T. Takeshita, ${ }^{140}$ M. Talby ${ }^{83}$ A. Talyshev, ${ }^{107}$ M. C. Tamsett, ${ }^{76}$ J. Tanaka,${ }^{155}$ R. Tanaka ${ }^{115}$ S. Tanaka ${ }^{131}$ S. Tanaka, ${ }^{66}$ Y. Tanaka, ${ }^{100}$ K. Tani ${ }^{67}$ G. P. Tappern, ${ }^{29}$ S. Tapprogge,${ }^{81}$ D. Tardif,${ }^{158}$ S. Tarem, ${ }^{152}$ F. Tarrade, ${ }^{24}$ G. F. Tartarelli, ${ }^{89 a}$ P. Tas,${ }^{126}$ M. Tasevsky, ${ }^{125}$ E. Tassi,${ }^{36 a, 36 b}$ M. Tatarkhanov,${ }^{14}$ C. Taylor, ${ }^{77}$ F. E. Taylor, ${ }^{92}$ G. Taylor,${ }^{137}$ G. N. Taylor,${ }^{86}$ R.P. Taylor, ${ }^{169}$ W. Taylor, ${ }^{159 b}$ M. Teixeira Dias Castanheira, ${ }^{75}$ P. Teixeira-Dias, ${ }^{76}$ K. K. Temming ${ }^{48}$ H. Ten Kate ${ }^{29}$ P. K. Teng, ${ }^{151}$ Y. D. Tennenbaum-Katan, ${ }^{152}$ S. Terada,${ }^{66}$ K. Terashi, ${ }^{155}$ J. Terron, ${ }^{80}$ M. Terwort, ${ }^{41, t}$ M. Testa, ${ }^{47}$ R. J. Teuscher, ${ }^{158,1}$ C. M. Tevlin, ${ }^{82}$ J. Thadome, ${ }^{174}$ J. Therhaag, ${ }^{20}$ T. Theveneaux-Pelzer, ${ }^{78}$ M. Thioye, ${ }^{175} \mathrm{~S}$. Thoma,${ }^{48}$ J. P. Thomas, ${ }^{17}$ E. N. Thompson, ${ }^{84}$ P. D. Thompson, ${ }^{17}$ P. D. Thompson, ${ }^{158}$ R. J. Thompson, ${ }^{82}$ A. S. Thompson, ${ }^{53}$

E. Thomson, ${ }^{120}$ R. P. Thun,${ }^{87}$ T. Tic, ${ }^{125}$ V. O. Tikhomirov,${ }^{94}$ Y. A. Tikhonov, ${ }^{107}$ C. J. W. P. Timmermans, ${ }^{104}$

P. Tipton, ${ }^{175}$ F. J. Tique Aires Viegas, ${ }^{29}$ S. Tisserant ${ }^{83}$ J. Tobias,${ }^{48}$ B. Toczek,${ }^{37}$ T. Todorov ${ }^{4}$ S. Todorova-Nova, ${ }^{161}$

B. Toggerson, ${ }^{163}$ J. Tojo, ${ }^{66}$ S. Tokár, ${ }^{144 a} \mathrm{~K}$. Tokunaga, ${ }^{67} \mathrm{~K}$. Tokushuku, ${ }^{66} \mathrm{~K}$. Tollefson, ${ }^{88} \mathrm{~L}$. Tomasek, ${ }^{125}$

M. Tomasek, ${ }^{125}$ M. Tomoto, ${ }^{101}$ D. Tompkins, ${ }^{6}$ L. Tompkins,${ }^{14} \mathrm{~K}$. Toms, ${ }^{103}$ A. Tonazzo, ${ }^{134 a, 134 b}$ G. Tong, ${ }^{32 a}$

A. Tonoyan, ${ }^{13}$ C. Topfel, ${ }^{16}$ N. D. Topilin, ${ }^{65}$ I. Torchiani, ${ }^{29}$ E. Torrence, ${ }^{114}$ E. Torró Pastor, ${ }^{167}$ J. Toth, ${ }^{83, c c}$ F. Touchard ${ }^{83}$ D. R. Tovey, ${ }^{139}$ D. Traynor, ${ }^{75}$ T. Trefzger, ${ }^{173}$ J. Treis,${ }^{20}$ L. Tremblet, ${ }^{29}$ A. Tricoli, ${ }^{29}$ I. M. Trigger,${ }^{159 a}$ S. Trincaz-Duvoid, ${ }^{78}$ T. N. Trinh,${ }^{78}$ M. F. Tripiana, ${ }^{70}$ N. Triplett, ${ }^{64}$ W. Trischuk, ${ }^{158}$ A. Trivedi, ${ }^{24, b b}$ B. Trocmé,${ }^{55}$ C. Troncon, ${ }^{89 \mathrm{a}}$ M. Trottier-McDonald, ${ }^{142}$ A. Trzupek, ${ }^{38}$ C. Tsarouchas, ${ }^{9}$ J. C-L. Tseng, ${ }^{118}$ M. Tsiakiris,,${ }^{105}$ P. V. Tsiareshka, ${ }^{90}$ D. Tsionou, ${ }^{139}$ G. Tsipolitis, ${ }^{9}$ V. Tsiskaridze, ${ }^{51}$ E. G. Tskhadadze,${ }^{51}$ I. I. Tsukerman,${ }^{95}$ V. Tsulaia, ${ }^{123} \mathrm{~J} .-\mathrm{W}$. Tsung, ${ }^{20} \mathrm{~S}$. Tsuno,${ }^{66} \mathrm{D}$. Tsybychev, ${ }^{148} \mathrm{~J}$. M. Tuggle,${ }^{30} \mathrm{M}$. Turala,${ }^{38} \mathrm{D}$. Turecek, ${ }^{127}$ I. Turk Cakir, ${ }^{3 e}$ E. Turlay, ${ }^{105}$ P. M. Tuts, ${ }^{34}$ M. S. Twomey, ${ }^{138}$ M. Tylmad, ${ }^{146 a, 146 b}$ M. Tyndel,,${ }^{129}$ D. Typaldos, ${ }^{17}$ H. Tyrvainen, ${ }^{29}$ E. Tzamarioudaki, ${ }^{9}$ G. Tzanakos, ${ }^{8}$ K. Uchida, ${ }^{20}$ I. Ueda, ${ }^{155}$ R. Ueno, ${ }^{28}$ M. Ugland, ${ }^{13}$ M. Uhlenbrock, ${ }^{20}$ M. Uhrmacher, ${ }^{54}$ F. Ukegawa, ${ }^{160}$ G. Unal, ${ }^{29}$ D. G. Underwood,${ }^{5}$ A. Undrus, ${ }^{24}$ G. Unel, ${ }^{163}$ Y. Unno, ${ }^{66}$ D. Urbaniec, ${ }^{34}$ E. Urkovsky, ${ }^{153}$ P. Urquijo, ${ }^{49, \text { hh }}$ P. Urrejola, ${ }^{31 a}$ G. Usai, ${ }^{7}$ M. Uslenghi, ${ }^{119 a, 119 b}$

L. Vacavant,${ }^{83}$ V. Vacek,${ }^{127}$ B. Vachon,${ }^{85}$ S. Vahsen, ${ }^{14}$ C. Valderanis,${ }^{99}$ J. Valenta, ${ }^{125}$ P. Valente, ${ }^{132 a}$ S. Valentinetti, ${ }^{19 a, 19 b}$ S. Valkar, ${ }^{126}$ E. Valladolid Gallego, ${ }^{167}$ S. Vallecorsa, ${ }^{152}$ J. A. Valls Ferrer, ${ }^{167}$ R. Van Berg, ${ }^{120}$ H. van der Graaf, ${ }^{105}$ E. van der Kraaij, ${ }^{105}$ E. van der Poel, ${ }^{105}$ D. van der Ster, ${ }^{29}$ B. Van Eijk, ${ }^{105}$ N. van Eldik, ${ }^{84}$ P. van Gemmeren, ${ }^{5}$ Z. van Kesteren, ${ }^{105}$ I. van Vulpen, ${ }^{105}$ W. Vandelli, ${ }^{29}$ G. Vandoni, ${ }^{29}$ A. Vaniachine, ${ }^{5}$ P. Vankov, ${ }^{73}$ F. Vannucci, ${ }^{78}$ F. Varela Rodriguez, ${ }^{29}$ R. Vari, ${ }^{132 a}$ E. W. Varnes, ${ }^{6}$ D. Varouchas, ${ }^{14}$ A. Vartapetian, ${ }^{7}$ K. E. Varvell, ${ }^{150}$

L. Vasilyeva, ${ }^{94}$ V. I. Vassilakopoulos ${ }^{56}$ F. Vazeille, ${ }^{33}$ G. Vegni, ${ }^{89 a, 89 b}$ J. J. Veillet, ${ }^{115}$ C. Vellidis, ${ }^{8}$ F. Veloso,${ }^{124 a}$ R. Veness, ${ }^{29}$ S. Veneziano, ${ }^{132 \mathrm{a}}$ A. Ventura,${ }^{72 a, 72 b}$ D. Ventura, ${ }^{138}$ S. Ventura,${ }^{47}$ M. Venturi, ${ }^{48} \mathrm{~N}$. Venturi, ${ }^{16}$ V. Vercesi, ${ }^{119 a}$ M. Verducci, ${ }^{138}$ W. Verkerke, ${ }^{105}$ J. C. Vermeulen, ${ }^{105}$ L. Vertogardov, ${ }^{118}$ M. C. Vetterli, ${ }^{142, f}$ I. Vichou, ${ }^{165}$ T. Vickey, ${ }^{145, \text { hh }}$ G. H. A. Viehhauser, ${ }^{118}$ S. Viel, ${ }^{168}$ M. Villa, ${ }^{19 a, 19 b}$ E. G. Villani, ${ }^{129}$ M. Villaplana Perez, ${ }^{167}$ E. Vilucchi, ${ }^{47}$ M. G. Vincter, ${ }^{28}$ E. Vinek, ${ }^{29}$ V. B. Vinogradov, ${ }^{65}$ M. Virchaux, ${ }^{136, a}$ S. Viret,${ }^{33}$ J. Virzi,${ }^{14}$ A. Vitale, ${ }^{19 a, 19 b}$ O. Vitells,${ }^{171}$ I. Vivarelli, ${ }^{48}$ F. Vives Vaque, ${ }^{11}$ S. Vlachos, ${ }^{9}$ M. Vlasak,${ }^{127}$ N. Vlasov,${ }^{20}$ 
A. Vogel, ${ }^{20}$ H. Vogt,${ }^{41}$ P. Vokac, ${ }^{127}$ M. Volpi,${ }^{11}$ G. Volpini, ${ }^{89 a}$ H. von der Schmitt, ${ }^{99}$ J. von Loeben,,${ }^{99}$ H. von Radziewski, ${ }^{48}$ E. von Toerne,${ }^{20}$ V. Vorobel, ${ }^{126}$ A. P. Vorobiev, ${ }^{128}$ V. Vorwerk, ${ }^{11}$ M. Vos,${ }^{167}$ R. Voss, ${ }^{29}$ T. T. Voss, ${ }^{174}$ J. H. Vossebeld, ${ }^{73}$ A. S. Vovenko, ${ }^{128}$ N. Vranjes, ${ }^{12 a}$ M. Vranjes Milosavljevic, ${ }^{12 a}$ V. Vrba, ${ }^{125}$ M. Vreeswijk, ${ }^{105}$ T. Vu Anh ${ }^{81}$ D. Vudragovic, ${ }^{12 a}$ R. Vuillermet, ${ }^{29}$ I. Vukotic, ${ }^{115}$ W. Wagner,${ }^{174}$ P. Wagner, ${ }^{120}$ H. Wahlen, ${ }^{174}$ J. Walbersloh, ${ }^{42}$ J. Walder, ${ }^{71}$ R. Walker, ${ }^{98}$ W. Walkowiak, ${ }^{141}$ R. Wall, ${ }^{175}$ P. Waller,${ }^{73}$ C. Wang, ${ }^{44}$ H. Wang, ${ }^{172}$ J. Wang, ${ }^{32 \mathrm{~d}}$ J. C. Wang, ${ }^{138}$ S. M. Wang, ${ }^{151}$ A. Warburton, ${ }^{85}$ C. P. Ward,${ }^{27}$ M. Warsinsky, ${ }^{48}$ R. Wastie, ${ }^{118}$ P. M. Watkins, ${ }^{17}$ A. T. Watson, ${ }^{17}$ M. F. Watson, ${ }^{17}$ G. Watts, ${ }^{138}$ S. Watts, ${ }^{82}$ A. T. Waugh,${ }^{150}$ B. M. Waugh,${ }^{77}$ M. Webel, ${ }^{48}$ J. Weber, ${ }^{42}$ M. Weber, ${ }^{129}$ M. S. Weber, ${ }^{16}$ P. Weber,${ }^{54}$ A. R. Weidberg, ${ }^{118}$ J. Weingarten, ${ }^{54}$ C. Weiser,${ }^{48}$ H. Wellenstein, ${ }^{22}$ H. P. Wellisch, ${ }^{159 a}$ P. S. Wells,${ }^{29}$ M. Wen, ${ }^{47}$ T. Wenaus,${ }^{24}$ S. Wendler, ${ }^{123}$ Z. Weng, ${ }^{151, w}$

T. Wengler, ${ }^{29}$ S. Wenig, ${ }^{29}$ N. Wermes, ${ }^{20}$ M. Werner, ${ }^{48}$ P. Werner, ${ }^{29}$ M. Werth ${ }^{163}$ U. Werthenbach, ${ }^{141}$ M. Wessels, ${ }^{58 a}$ K. Whalen ${ }^{28}$ S. J. Wheeler-Ellis, ${ }^{163}$ S. P. Whitaker, ${ }^{21}$ A. White, ${ }^{7}$ M. J. White, ${ }^{27}$ S. White, ${ }^{24}$ S. R. Whitehead,${ }^{118}$ D. Whiteson, ${ }^{163}$ D. Whittington, ${ }^{61}$ F. Wicek, ${ }^{115}$ D. Wicke, ${ }^{81}$ F. J. Wickens, ${ }^{129}$ W. Wiedenmann, ${ }^{172}$ M. Wielers, ${ }^{129}$ P. Wienemann, ${ }^{20}$ C. Wiglesworth, ${ }^{73}$ L. A. M. Wiik ${ }^{48}$ A. Wildauer, ${ }^{167}$ M. A. Wildt, ${ }^{41, t}$ I. Wilhelm, ${ }^{126}$ H. G. Wilkens, ${ }^{29}$ J.Z. Will, ${ }^{98}$ E. Williams, ${ }^{34}$ H. H. Williams, ${ }^{120}$ W. Willis, ${ }^{34}$ S. Willocq, ${ }^{84}$ J. A. Wilson, ${ }^{17}$ M. G. Wilson, ${ }^{143}$ A. Wilson, ${ }^{87}$ I. Wingerter-Seez, ${ }^{4}$ S. Winkelmann, ${ }^{48}$ F. Winklmeier, ${ }^{29}$ M. Wittgen, ${ }^{143}$ E. Woehrling, ${ }^{17}$ M. W. Wolter, ${ }^{38}$ H. Wolters, ${ }^{124 a, i}$ B. K. Wosiek, ${ }^{38}$ J. Wotschack, ${ }^{29}$ M. J. Woudstra, ${ }^{84}$ K. Wraight,${ }^{53}$ C. Wright,${ }^{53}$ D. Wright, ${ }^{143}$ B. Wrona, ${ }^{73}$ S. L. Wu, ${ }^{172}$ X. Wu, ${ }^{49}$ J. Wuestenfeld ${ }^{42}$ E. Wulf, ${ }^{34}$ R. Wunstorf, ${ }^{42}$ B. M. Wynne, ${ }^{45}$ L. Xaplanteris, ${ }^{9}$ S. Xella, ${ }^{35}$ S. Xie, ${ }^{48}$ Y. Xie,${ }^{32 a}$ C. Xu, ${ }^{32 b}$ D. Xu, ${ }^{139}$ G. Xu, ${ }^{32 a}$ N. Xu, ${ }^{172}$ B. Yabsley, ${ }^{150}$ M. Yamada, ${ }^{66}$ A. Yamamoto, ${ }^{66}$ K. Yamamoto, ${ }^{64}$ S. Yamamoto, ${ }^{155}$ T. Yamamura, ${ }^{155}$ J. Yamaoka, ${ }^{44}$ T. Yamazaki, ${ }^{155}$ Y. Yamazaki, ${ }^{67} \mathrm{Z}$. Yan, ${ }^{21}$ H. Yang, ${ }^{87}$ S. Yang, ${ }^{18}$ U. K. Yang, ${ }^{82}$ Y. Yang, ${ }^{61}$ Y. Yang, ${ }^{32 a}$ Z. Yang,,${ }^{146 a, 146 b}$ W-M. Yao, ${ }^{14}$ Y. Yao, ${ }^{14}$ Y. Yasu, ${ }^{66}$ J. Ye, ${ }^{39}$ S. Ye, ${ }^{24}$ M. Yilmaz, ${ }^{3 c}$ R. Yoosoofmiya, ${ }^{123}$ K. Yorita, ${ }^{170}$ R. Yoshida, ${ }^{5}$ C. Young, ${ }^{143}$ S. P. Youssef,${ }^{21}$ D. Yu, ${ }^{24}$ J. Yu, ${ }^{7}$ J. Yu, ${ }^{32 c, i i}$ J. Yuan, ${ }^{99}$ L. Yuan, ${ }^{32 a, j j}$ A. Yurkewicz, ${ }^{148}$ V. G. Zaets, ${ }^{128}$ R. Zaidan, ${ }^{63}$ A. M. Zaitsev, ${ }^{128}$ Z. Zajacova, ${ }^{29}$ Yo. K. Zalite, ${ }^{121}$ V. Zambrano, ${ }^{47}$ L. Zanello, ${ }^{132 a, 132 b}$ P. Zarzhitsky, ${ }^{39}$ A. Zaytsev, ${ }^{107}$ M. Zdrazil ${ }^{14}$ C. Zeitnitz, ${ }^{174}$ M. Zeller, ${ }^{175}$ P. F. Zema, ${ }^{29}$ A. Zemla, ${ }^{38}$ C. Zendler ${ }^{20}$ A. V. Zenin, ${ }^{128}$ O. Zenin, ${ }^{128}$ T. Zenis, ${ }^{144 a}$ Z. Zenonos, ${ }^{122 a, 122 b}$ S. Zenz, ${ }^{14}$ D. Zerwas, ${ }^{115}$ G. Zevi della Porta, ${ }^{57}$ Z. Zhan, ${ }^{32 \mathrm{~d}}$ H. Zhang, ${ }^{83}$ J. Zhang, ${ }^{5}$ Q. Zhang, ${ }^{5}$ X. Zhang, ${ }^{32 \mathrm{~d}}$ L. Zhao, ${ }^{108}$ T. Zhao, ${ }^{138}$ Z. Zhao, ${ }^{32 \mathrm{~b}}$ A. Zhemchugov, ${ }^{65}$ S. Zheng, ${ }^{32 \mathrm{a}}$ J. Zhong, ${ }^{151, k \mathrm{k}}$ B. Zhou, ${ }^{87}$ N. Zhou, ${ }^{163}$ Y. Zhou, ${ }^{151}$ C. G. Zhu, ${ }^{32 \mathrm{~d}}$ H. Zhu ${ }^{41}$ Y. Zhu, ${ }^{172}$ X. Zhuang, ${ }^{98}$ V. Zhuravlov, ${ }^{99}$ B. Zilka, ${ }^{144 a}$ R. Zimmermann,${ }^{20}$ S. Zimmermann, ${ }^{20}$ S. Zimmermann ${ }^{48}$ M. Ziolkowski, ${ }^{141}$ R. Zitoun ${ }^{4}$ L. Živković, ${ }^{34}$ V. V. Zmouchko, ${ }^{128, a}$ G. Zobernig, ${ }^{172}$ A. Zoccoli, ${ }^{19 a, 19 b}$ Y. Zolnierowski, ${ }^{4}$ A. Zsenei, ${ }^{29}$ M. zur Nedden, ${ }^{15}$ and V. Zutshi ${ }^{106}$

(ATLAS Collaboration)

\footnotetext{
${ }^{1}$ University at Albany, 1400 Washington Avenue, Albany, New York 12222, USA

${ }^{2}$ University of Alberta, Department of Physics, Centre for Particle Physics, Edmonton, Alberta T6G 2G7, Canada

${ }^{3 a}$ Ankara University, Faculty of Sciences, Department of Physics, TR 061000 Tandogan, Ankara, Turkey;

${ }^{3 b}$ Dumlupinar University, Faculty of Arts and Sciences, Department of Physics, Kutahya, Turkey;

${ }^{3 c}$ Gazi University, Faculty of Arts and Sciences, Department of Physics, 06500, Teknikokullar, Ankara, Turkey;
}

${ }^{3 \mathrm{~d}}$ TOBB University of Economics and Technology, Faculty of Arts and Sciences, Division of Physics, 06560, Sogutozu, Ankara, Turkey; ${ }^{3 e}$ Turkish Atomic Energy Authority, 06530, Lodumlu, Ankara, Turkey

${ }^{4} L A P P$, Université de Savoie, CNRS/IN2P3, Annecy-le-Vieux, France

${ }^{5}$ Argonne National Laboratory, High Energy Physics Division, 9700 S. Cass Avenue, Argonne, Illinois 60439, USA

${ }^{6}$ University of Arizona, Department of Physics, Tucson, Arizona 85721, USA

${ }^{7}$ The University of Texas at Arlington, Department of Physics, Box 19059, Arlington, Texas 76019, USA

${ }^{8}$ University of Athens, Nuclear and Particle Physics, Department of Physics, Panepistimiopouli, Zografou, GR 15771 Athens, Greece

${ }^{9}$ National Technical University of Athens, Physics Department, 9-Iroon Polytechniou, GR 15780 Zografou, Greece

${ }^{10}$ Institute of Physics, Azerbaijan Academy of Sciences, H. Javid Avenue 33, AZ 143 Baku, Azerbaijan

${ }^{11}$ Institut de Física d'Altes Energies, IFAE, Edifici Cn, Universitat Autònoma de Barcelona, ES-08193 Bellaterra (Barcelona), Spain

${ }^{12 a}$ University of Belgrade, Institute of Physics, P.O. Box 57, 11001 Belgrade, Serbia;

${ }^{12 \mathrm{~b}}$ Vinca Institute of Nuclear Sciences, M. Petrovica Alasa 12-14, 11000 Belgrade, Serbia

${ }^{13}$ University of Bergen, Department for Physics and Technology, Allegaten 55, NO-5007 Bergen, Norway

${ }^{14}$ Lawrence Berkeley National Laboratory and University of California, Physics Division, MS50B-6227, 1 Cyclotron Road, Berkeley, California 94720, USA

${ }^{15}$ Humboldt University, Institute of Physics, Berlin, Newtonstrasse 15, D-12489 Berlin, Germany 
${ }^{16}$ University of Bern, Albert Einstein Center for Fundamental Physics, Laboratory for High Energy Physics, Sidlerstrasse 5, CH-3012 Bern, Switzerland

${ }^{17}$ University of Birmingham, School of Physics and Astronomy, Edgbaston, Birmingham B15 2TT, United Kingdom

${ }^{18 a}$ Bogazici University, Faculty of Sciences, Department of Physics, TR-80815 Bebek-Istanbul, Turkey;

${ }^{18 \mathrm{~b}}$ Dogus University, Faculty of Arts and Sciences, Department of Physics, 34722, Kadikoy, Istanbul, Turkey;

${ }^{18 c}$ Gaziantep University, Faculty of Engineering, Department of Physics Engineering, 27310, Sehitkamil, Gaziantep, Turkey;

${ }^{19 a}$ INFN Sezione di Bologna;

${ }^{19 b}$ Università di Bologna, Dipartimento di Fisica, viale C. Berti Pichat, 6/2, IT-40127 Bologna, Italy

${ }^{20}$ University of Bonn, Physikalisches Institut, Nussallee 12, D-53115 Bonn, Germany

${ }^{21}$ Boston University, Department of Physics, 590 Commonwealth Avenue, Boston, Massachusetts 02215, USA

${ }^{22}$ Brandeis University, Department of Physics, MS057, 415 South Street, Waltham, Massachusetts 02454, USA

${ }^{23}$ Universidade Federal do Rio De Janeiro, COPPE/EE/IF, Caixa Postal 68528, Ilha do Fundao,

BR-21945-970 Rio de Janeiro, Brazil;

${ }^{24}$ Brookhaven National Laboratory, Physics Department, Building 510A, Upton, New York 11973, USA

${ }^{25 \mathrm{a}}$ National Institute of Physics and Nuclear Engineering, Bucharest-Magurele, Strasse Atomistilor 407, P.O. Box MG-6, R-077125, Romania;

${ }^{25 b}$ University Politehnica Bucharest, Rectorat - AN 001, 313 Splaiul Independentei, sector 6, 060042 Bucuresti, Romania;

${ }^{26}$ Universidad de Buenos Aires, FCEyN, Departamento Fisica, Pab I - C. Universitaria, 1428 Buenos Aires, Argentina

${ }^{27}$ University of Cambridge, Cavendish Laboratory, JJ Thomson Avenue, Cambridge CB3 OHE, United Kingdom

${ }^{28}$ Carleton University, Department of Physics, 1125 Colonel By Drive, Ottawa, Ontario K1S 5B6, Canada

${ }^{29} \mathrm{CERN}, \mathrm{CH}-1211$ Geneva 23, Switzerland

${ }^{30}$ University of Chicago, Enrico Fermi Institute, 5640 S. Ellis Avenue, Chicago, IL 60637, USA

${ }^{31 a}$ Pontificia Universidad Católica de Chile, Facultad de Fisica, Departamento de Fisica, Avenida Vicuna Mackenna 4860, San Joaquin, Santiago, Chile;

${ }^{31 \mathrm{~b}}$ Universidad Técnica Federico Santa María, Departamento de Física, Avenida Espãna 1680, Casilla 110-V, Valparaíso, Chile

${ }^{32 \mathrm{a}}$ Institute of High Energy Physics, Chinese Academy of Sciences, P.O. Box 918, 19 Yuquan Road, Shijing Shan District, CN - Beijing 100049, China,

${ }^{32 \mathrm{~b}}$ University of Science and Technology of China (USTC), Department of Modern Physics, Hefei, CN - Anhui 230026, China;

${ }^{32 \mathrm{c}}$ Nanjing University, Department of Physics, Nanjing, CN - Jiangsu 210093, China;

${ }^{32 \mathrm{~d}}$ Shandong University, High Energy Physics Group, Jinan, CN - Shandong 250100, China

${ }^{33}$ Laboratoire de Physique Corpusculaire, Clermont Université, Université Blaise Pascal, CNRS/IN2P3, FR-63177 Aubiere Cedex, France

${ }^{34}$ Columbia University, Nevis Laboratory, 136 South Broadway, Irvington, New York 10533, USA

${ }^{35}$ University of Copenhagen, Niels Bohr Institute, Blegdamsvej 17, DK-2100 Kobenhavn 0, Denmark ${ }^{36 a}$ INFN Gruppo Collegato di Cosenza;

${ }^{36 \mathrm{~b}}$ Università della Calabria, Dipartimento di Fisica, IT-87036 Arcavacata di Rende, Italy

${ }^{37}$ Faculty of Physics and Applied Computer Science of the AGH-University of Science and Technology (FPACS, AGH-UST), al. Mickiewicza 30, PL-30059 Cracow, Poland

${ }^{38}$ The Henryk Niewodniczanski Institute of Nuclear Physics, Polish Academy of Sciences, ul. Radzikowskiego 152, PL-31342 Krakow, Poland

${ }^{39}$ Southern Methodist University, Physics Department, 106 Fondren Science Building, Dallas, Texas 75275-0175, USA

${ }^{40}$ University of Texas at Dallas, 800 West Campbell Road, Richardson, Texas 75080-3021, USA

${ }^{41}$ DESY, Notkestrasse 85, D-22603 Hamburg and Platanenallee 6, D-15738 Zeuthen, Germany

${ }^{42}$ TU Dortmund, Experimentelle Physik IV, DE-44221 Dortmund, Germany

${ }^{43}$ Technical University Dresden, Institut für Kern- und Teilchenphysik, Zellescher Weg 19, D-01069 Dresden, Germany

${ }^{44}$ Duke University, Department of Physics, Durham, North Carolina 27708, USA

${ }^{45}$ University of Edinburgh, School of Physics and Astronomy, James Clerk Maxwell Building, The Kings Buildings, Mayfield Road, Edinburgh EH9 3JZ, United Kingdom

${ }^{46}$ Fachhochschule Wiener Neustadt, Johannes Gutenbergstrasse 3 AT-2700 Wiener Neustadt, Austria

${ }^{47}$ INFN Laboratori Nazionali di Frascati, via Enrico Fermi 40, IT-00044 Frascati, Italy

${ }^{48}$ Albert-Ludwigs-Universität, Fakultät für Mathematik und Physik, Hermann-Herder Strasse 3, D-79104 Freiburg i.Br., Germany

${ }^{49}$ Université de Genève, Section de Physique, 24 rue Ernest Ansermet, CH-1211 Geneve 4, Switzerland

${ }^{50 a}$ INFN Sezione di Genova;

${ }^{50 \mathrm{~b}}$ Università di Genova, Dipartimento di Fisica, via Dodecaneso 33, IT-16146 Genova, Italy

${ }^{51}$ Institute of Physics of the Georgian Academy of Sciences, 6 Tamarashvili Street, GE-380077 Tbilisi;

Tbilisi State University, HEP Institute, University Street 9, GE-380086 Tbilisi, Georgia

${ }^{52}$ Justus-Liebig-Universität Giessen, II Physikalisches Institut, Heinrich-Buff Ring 16, D-35392 Giessen, Germany

${ }^{53}$ University of Glasgow, Department of Physics and Astronomy, Glasgow G12 8QQ, United Kingdom

${ }^{54}$ Georg-August-Universität, II. Physikalisches Institut, Friedrich-Hund Platz, 1, D-37077 Göttingen, Germany

${ }^{55}$ Laboratoire de Physique Subatomique et de Cosmologie, CNRS/IN2P3, Université Joseph Fourier, INPG, 53 avenue des Martyrs, FR-38026 Grenoble Cedex, France 
${ }^{56}$ Hampton University, Department of Physics, Hampton, Virginia 23668, USA

${ }^{57}$ Harvard University, Laboratory for Particle Physics and Cosmology, 18 Hammond Street, Cambridge, Massachusetts 02138, USA

${ }^{58}{ }^{5}$ Ruprecht-Karls-Universität Heidelberg: Kirchhoff-Institut für Physik, Im Neuenheimer Feld 227, D-69120 Heidelberg, Germany;

${ }^{58 \mathrm{~b}}$ Physikalisches Institut, Philosophenweg 12, D-69120 Heidelberg, Germany;

${ }^{58 c}$ ZITI Ruprecht-Karls-University Heidelberg, Lehrstuhl für Informatik V, B6, 23-29, DE-68131 Mannheim, Germany

${ }^{59}$ Hiroshima University, Faculty of Science, 1-3-1 Kagamiyama, Higashihiroshima-shi, JP - Hiroshima 739-8526, Japan

${ }^{60}$ Hiroshima Institute of Technology, Faculty of Applied Information Science, 2-1-1 Miyake Saeki-ku, Hiroshima-shi, JP - Hiroshima 731-5193, Japan

${ }^{61}$ Indiana University, Department of Physics, Swain Hall West 117, Bloomington, Indiana 47405-7105, USA

${ }^{62}$ Institut für Astro- und Teilchenphysik, Technikerstrasse 25, A-6020 Innsbruck, Austria

${ }^{63}$ University of Iowa, 203 Van Allen Hall, Iowa City, Iowa 52242-1479, USA

${ }^{64}$ Iowa State University, Department of Physics and Astronomy, Ames High Energy Physics Group, Ames, Iowa 50011-3160, USA

${ }^{65}$ Joint Institute for Nuclear Research, JINR Dubna, RU-141 980 Moscow Region, Russia

${ }^{66}$ KEK, High Energy Accelerator Research Organization, 1-1 Oho, Tsukuba-shi, Ibaraki-ken 305-0801, Japan

${ }^{67}$ Kobe University, Graduate School of Science, 1-1 Rokkodai-cho, Nada-ku, JP Kobe 657-8501, Japan

${ }^{68}$ Kyoto University, Faculty of Science, Oiwake-cho, Kitashirakawa, Sakyou-ku, Kyoto-shi, JP - Kyoto 606-8502, Japan

${ }^{69}$ Kyoto University of Education, 1 Fukakusa, Fujimori, fushimi-ku, Kyoto-shi, JP - Kyoto 612-8522, Japan

${ }^{70}$ Universidad Nacional de La Plata, FCE, Departamento de Física, IFLP (CONICET-UNLP), C.C. 67, 1900 La Plata, Argentina

${ }^{71}$ Lancaster University, Physics Department, Lancaster LA1 4YB, United Kingdom

${ }^{72 \mathrm{a}}$ INFN Sezione di Lecce;

${ }^{72 b}$ Università del Salento, Dipartimento di Fisica, Via Arnesano IT-73100 Lecce, Italy

${ }^{73}$ University of Liverpool, Oliver Lodge Laboratory, P.O. Box 147, Oxford Street, Liverpool L69 3BX, United Kingdom

${ }^{74}$ Jožef Stefan Institute and University of Ljubljana, Department of Physics, SI-1000 Ljubljana, Slovenia

${ }^{75}$ Queen Mary University of London, Department of Physics, Mile End Road, London E1 4NS, United Kingdom

${ }^{76}$ Royal Holloway, University of London, Department of Physics, Egham Hill, Egham, Surrey TW20 OEX, United Kingdom

${ }^{77}$ University College London, Department of Physics and Astronomy, Gower Street, London WC1E 6BT, United Kingdom

${ }^{78}$ Laboratoire de Physique Nucléaire et de Hautes Energies, Université Pierre et Marie Curie (Paris 6),

Université Denis Diderot (Paris-7), CNRS/IN2P3, Tour 33, 4 place Jussieu, FR-75252 Paris Cedex 05, France

${ }^{79}$ Lunds Universitet, Naturvetenskapliga fakulteten, Fysiska institutionen, Box 118, SE-221 00 Lund, Sweden

${ }^{80}$ Universidad Autonoma de Madrid, Facultad de Ciencias, Departamento de Fisica Teorica, ES-28049 Madrid, Spain

${ }^{81}$ Universität Mainz, Institut für Physik, Staudinger Weg 7, DE-55099 Mainz, Germany

${ }^{82}$ University of Manchester, School of Physics and Astronomy, Manchester M13 9PL, United Kingdom

${ }^{83}$ CPPM, Aix-Marseille Université, CNRS/IN2P3, Marseille, France

${ }^{84}$ University of Massachusetts, Department of Physics, 710 North Pleasant Street, Amherst, Massachusetts 01003, USA

${ }^{85}$ McGill University, High Energy Physics Group, 3600 University Street, Montreal, Quebec H3A 2T8, Canada

${ }^{86}$ University of Melbourne, School of Physics, AU - Parkville, Victoria 3010, Australia

${ }^{87}$ The University of Michigan, Department of Physics, 2477 Randall Laboratory, 500 East University, Ann Arbor, Michigan 48109-1120, USA

${ }^{88}$ Michigan State University, Department of Physics and Astronomy, High Energy Physics Group, East Lansing, Michigan 48824-2320, USA

${ }^{89 a}$ INFN Sezione di Milano;

${ }^{89 \mathrm{~b}}$ Università di Milano, Dipartimento di Fisica, via Celoria 16, IT-20133 Milano, Italy

${ }^{90}$ B. I. Stepanov Institute of Physics, National Academy of Sciences of Belarus, Independence Avenue 68, Minsk 220072, Belarus

${ }^{91}$ National Scientific and Educational Centre for Particle and High Energy Physics, NC PHEP BSU,

M. Bogdanovich Street 153, Minsk 220040, Belarus

${ }^{92}$ Massachusetts Institute of Technology, Department of Physics, Room 24-516, Cambridge, Massachusetts 02139, USA

${ }^{93}$ University of Montreal, Group of Particle Physics, C.P. 6128, Succursale Centre-Ville, Montreal, Quebec, H3C 3J7, Canada

${ }^{94}$ P. N. Lebedev Institute of Physics, Academy of Sciences, Leninsky pr. 53, RU-117 924 Moscow, Russia

${ }^{95}$ Institute for Theoretical and Experimental Physics (ITEP), B. Cheremushkinskaya ul. 25, RU 117218 Moscow, Russia

${ }^{96}$ Moscow Engineering and Physics Institute (MEPhI), Kashirskoe Shosse 31, RU-115409 Moscow, Russia

${ }^{97}$ Lomonosov Moscow State University, Skobeltsyn Institute of Nuclear Physics (MSU SINP), 1(2), Leninskie gory, GSP-1, Moscow 119991 Russian Federation, Russia

${ }^{98}$ Ludwig-Maximilians-Universität München, Fakultät für Physik, Am Coulombwall 1, DE-85748 Garching, Germany

${ }^{99}$ Max-Planck-Institut für Physik (Werner-Heisenberg-Institut), Föhringer Ring 6, 80805 München, Germany

${ }^{100}$ Nagasaki Institute of Applied Science, 536 Aba-machi, JP Nagasaki 851-0193, Japan

${ }^{101}$ Nagoya University, Graduate School of Science, Furo-Cho, Chikusa-ku, Nagoya, 464-8602, Japan

${ }^{102 a}$ INFN Sezione di Napoli;

${ }^{102 \mathrm{~b}}$ Università di Napoli, Dipartimento di Scienze Fisiche, Complesso Universitario di Monte Sant'Angelo, via Cinthia, IT-80126 Napoli, Italy

${ }^{103}$ University of New Mexico, Department of Physics and Astronomy, MSC07 4220, Albuquerque, New Mexico 87131 , USA 
${ }^{104}$ Radboud University Nijmegen/NIKHEF, Department of Experimental High Energy Physics, Heyendaalseweg 135 , NL-6525 AJ, Nijmegen, The Netherlands

${ }^{105}$ Nikhef National Institute for Subatomic Physics and University of Amsterdam, Science Park 105, 1098 XG Amsterdam, The Netherlands

${ }^{106}$ Department of Physics, Northern Illinois University, LaTourette Hall Normal Road, DeKalb, Illinois 60115, USA

${ }^{107}$ Budker Institute of Nuclear Physics (BINP), RU - Novosibirsk 630 090, Russia

${ }^{108}$ New York University, Department of Physics, 4 Washington Place, New York, New York 10003, USA

${ }^{109}$ Ohio State University, 191 West Woodruff Avenue, Columbus, Ohio 43210-1117, USA

${ }^{110}$ Okayama University, Faculty of Science, Tsushimanaka 3-1-1, Okayama 700-8530, Japan

${ }^{111}$ University of Oklahoma, Homer L. Dodge Department of Physics and Astronomy, 440 West Brooks, Room 100, Norman, Oklahoma 73019-0225, USA

${ }^{112}$ Oklahoma State University, Department of Physics, 145 Physical Sciences Building, Stillwater, Oklahoma 74078-3072, USA

${ }^{113}$ Palacký University, 17.listopadu 50a, 77207 Olomouc, Czech Republic

${ }^{114}$ University of Oregon, Center for High Energy Physics, Eugene, Oregon 97403-1274, USA

${ }^{115}$ LAL, Université Paris-Sud, IN2P3/CNRS, Orsay, France

${ }^{116}$ Osaka University, Graduate School of Science, Machikaneyama-machi 1-1, Toyonaka, Osaka 560-0043, Japan

${ }^{117}$ University of Oslo, Department of Physics, P.O. Box 1048, Blindern, NO-0316 Oslo 3, Norway

${ }^{118}$ Oxford University, Department of Physics, Denys Wilkinson Building, Keble Road, Oxford OX1 3RH, United Kingdom

${ }^{119 a}$ INFN Sezione di Pavia;

${ }^{119 b}$ Università di Pavia, Dipartimento di Fisica Nucleare e Teorica, Via Bassi 6, IT-27100 Pavia, Italy

${ }^{120}$ University of Pennsylvania, Department of Physics, High Energy Physics Group, 209 S. 33rd Street,

Philadelphia, Pennsylvania 19104, USA

${ }^{121}$ Petersburg Nuclear Physics Institute, RU-188 300 Gatchina, Russia

${ }^{122 a}$ INFN Sezione di Pisa;

${ }^{122 b}$ Università di Pisa, Dipartimento di Fisica E. Fermi, Largo B. Pontecorvo 3, IT-56127 Pisa, Italy

${ }^{123}$ University of Pittsburgh, Department of Physics and Astronomy, 3941 O'Hara Street, Pittsburgh, Pennsylvania 15260, USA

${ }^{124 a}$ Laboratorio de Instrumentacao e Fisica Experimental de Particulas-LIP, Avenida Elias Garcia 14-1, PT-1000-149 Lisboa, Portugal;

${ }^{124 b}$ Universidad de Granada, Departamento de Fisica Teorica y del Cosmos and CAFPE, E-18071 Granada, Spain

${ }^{125}$ Institute of Physics, Academy of Sciences of the Czech Republic, Na Slovance 2, CZ-18221 Praha 8, Czech Republic

${ }^{126}$ Charles University in Prague, Faculty of Mathematics and Physics, Institute of Particle and Nuclear Physics,

$V$ Holesovickach 2, CZ-18000 Praha 8, Czech Republic

${ }^{127}$ Czech Technical University in Prague, Zikova 4, CZ-166 35 Praha 6, Czech Republic

${ }^{128}$ State Research Center Institute for High Energy Physics, Moscow Region, 142281, Protvino, Pobeda street, 1, Russia

${ }^{129}$ Rutherford Appleton Laboratory, Science and Technology Facilities Council, Harwell Science and Innovation Campus, Didcot OX11 0QX, United Kingdom

${ }^{130}$ University of Regina, Physics Department, Canada

${ }^{131}$ Ritsumeikan University, Noji Higashi 1 chome 1-1, JP - Kusatsu, Shiga 525-8577, Japan

${ }^{132 \mathrm{a}}$ INFN Sezione di Roma I;

${ }^{132 b}$ Università La Sapienza, Dipartimento di Fisica, Piazzale A. Moro 2, IT-00185 Roma, Italy

${ }^{133 a}$ INFN Sezione di Roma Tor Vergata;

${ }^{133 b}$ Università di Roma Tor Vergata, Dipartimento di Fisica, via della Ricerca Scientifica, IT-00133 Roma, Italy ${ }^{134 a}$ INFN Sezione di Roma Tre;

${ }^{134 b}$ Università Roma Tre, Dipartimento di Fisica, via della Vasca Navale 84, IT-00146 Roma, Italy

${ }^{135 a}$ Réseau Universitaire de Physique des Hautes Energies (RUPHE): Université Hassan II, Faculté des Sciences Ain Chock, B.P. 5366, MA - Casablanca, Morocco;

${ }^{135 \mathrm{~b}}$ Université Mohamed Premier, LPTPM, Faculté des Sciences, B.P. 717, Boulevard Mohamed VI, 60000, Oujda, Morocco;

${ }^{135 \mathrm{c}}$ Université Mohammed V, Faculté des Sciences, 4 Avenue Ibn Battouta, B.P. 1014 RP, 10000 Rabat, Morocco

${ }^{136}$ CEA, DSM/IRFU, Centre d'Etudes de Saclay, FR-91191 Gif-sur-Yvette, France

${ }^{137}$ University of California Santa Cruz, Santa Cruz Institute for Particle Physics (SCIPP), Santa Cruz, California 95064, USA

${ }^{138}$ University of Washington, Seattle, Department of Physics, Box 351560, Seattle, Washington 98195-1560, USA

${ }^{139}$ University of Sheffield, Department of Physics and Astronomy, Hounsfield Road, Sheffield S3 7RH, United Kingdom

${ }^{140}$ Shinshu University, Department of Physics, Faculty of Science, 3-1-1 Asahi, Matsumoto-shi, JP - Nagano 390-8621, Japan

${ }^{141}$ Universität Siegen, Fachbereich Physik, D 57068 Siegen, Germany

${ }^{142}$ Simon Fraser University, Department of Physics, 8888 University Drive, CA - Burnaby, British Columbia V5A 1S6, Canada

${ }^{143}$ SLAC National Accelerator Laboratory, Stanford, California 94309, USA

${ }^{144 a}$ Comenius University, Faculty of Mathematics, Physics and Informatics, Mlynska dolina F2, SK-84248 Bratislava, Slovak Republic;

${ }^{144 \mathrm{~b}}$ Institute of Experimental Physics of the Slovak Academy of Sciences, Department of Subnuclear Physics, Watsonova 47, SK-04353 Kosice, Slovak Republic

${ }^{145}$ School of Physics, University of the Witwatersrand, Private Bag 3, Wits 2050, Johannesburg, South Africa 


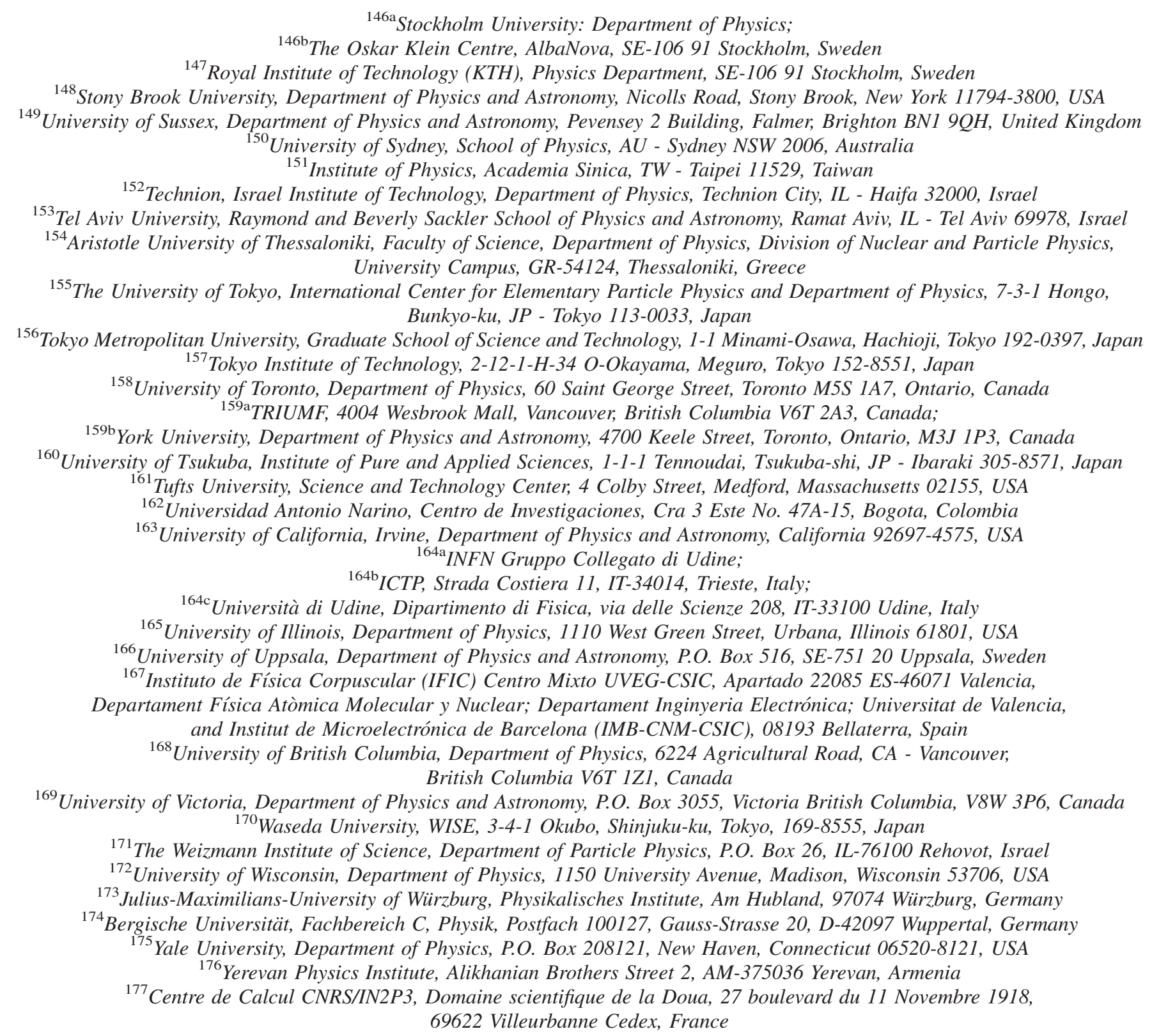

${ }^{\text {a } D e c e a s e d . ~}$

${ }^{\mathrm{b}}$ Also at LIP, Portugal.

${ }^{\mathrm{c}}$ Present address: FermiLab, USA.

${ }^{\mathrm{d}}$ Also at Faculdade de Ciencias, Universidade de Lisboa, Portugal.

${ }^{\mathrm{e}}$ Also at CPPM, Marseille, France.

${ }^{\mathrm{f}}$ Also at TRIUMF, Vancouver, Canada.

${ }^{\mathrm{g}}$ Also at FPACS, AGH-UST, Cracow, Poland.

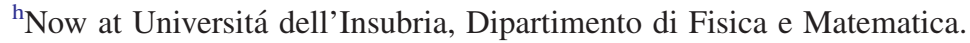

${ }^{i}$ Also at Department of Physics, University of Coimbra, Portugal.

${ }^{\mathrm{j}}$ Present address: CERN.

${ }^{\mathrm{k}}$ Also at Università di Napoli Parthenope, Napoli, Italy.

${ }^{1}$ Also at Institute of Particle Physics (IPP), Canada.

${ }^{\mathrm{m}}$ Also at Louisiana Tech University, Ruston, LA, USA.

${ }^{\mathrm{n}}$ Also at Universidade de Lisboa, Portugal.

${ }^{\circ}$ Also at California State University, Fresno, USA.

${ }^{\mathrm{p}}$ Present address: Istituto Universitario di Studi Superiori IUSS, Pavia, Italy. 
${ }^{\mathrm{q}}$ Also at Faculdade de Ciencias, Universidade de Lisboa, Portugal and at Centro de Fisica Nuclear da Universidade de Lisboa, Portugal.

${ }^{\mathrm{r}}$ Also at California Institute of Technology, Pasadena, CA, USA.

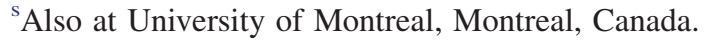

${ }^{\mathrm{t}}$ Also at Institut für Experimentalphysik, Universität Hamburg, Hamburg, Germany.

${ }^{\text {u}}$ Present address: Chonnam National University, Chonnam, Korea 500-757.

${ }^{v}$ Also at Petersburg Nuclear Physics Institute, Gatchina, Russia.

${ }^{w}$ Also at School of Physics and Engineering, Sun Yat-sen University, China.

${ }^{\mathrm{x}}$ Also at School of Physics, Shandong University, Jinan, China.

${ }^{\mathrm{y}}$ Also at Rutherford Appleton Laboratory, Didcot, United Kingdom.

${ }^{\mathrm{z}}$ Present address: KEK.

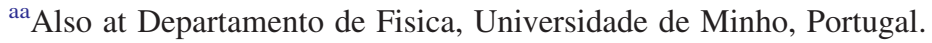

${ }^{\mathrm{bb}}$ Also at University of South Carolina, Columbia, SC, USA.

${ }^{\mathrm{cc}}$ Also at KFKI Research Institute for Particle and Nuclear Physics, Budapest, Hungary.

${ }^{\mathrm{dd}}$ Also at University of South Carolina, Department of Physics and Astronomy, 700 S. Main Street, Columbia, SC 29208 , USA.

${ }^{e e}$ Now at TRIUMF, Vancouver, Canada.

${ }^{\mathrm{ff}}$ Also at Institute of Physics, Jagiellonian University, Cracow, Poland.

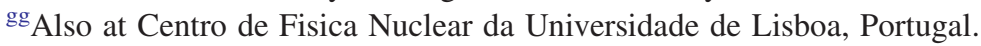

${ }^{\text {hh }}$ Also at Oxford University, Department of Physics, Denys Wilkinson Building, Keble Road, Oxford OX1 3RH, United Kingdom.

${ }^{\mathrm{ii}} \mathrm{Also}$ at CEA.

${ }^{\mathrm{jj}} \mathrm{Also}$ at LPNHE, Paris, France.

${ }^{\mathrm{kk}}$ Also at Nanjing University, China. 